\title{
Search for a heavy top-quark partner in final states with two leptons with the ATLAS detector at the LHC
}

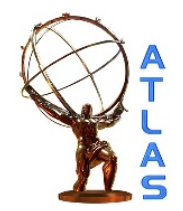

\section{The ATLAS collaboration}

\author{
E-mail: atlas.publications@cern.ch
}

ABSTRACT: The results of a search for direct pair production of heavy top-quark partners in $4.7 \mathrm{fb}^{-1}$ of integrated luminosity from $p p$ collisions at $\sqrt{s}=7 \mathrm{TeV}$ collected by the ATLAS detector at the LHC are reported. Heavy top-quark partners decaying into a top quark and a neutral non-interacting particle are searched for in events with two leptons in the final state. No excess above the Standard Model expectation is observed. Limits are placed on the mass of a supersymmetric scalar top and of a spin- $1 / 2$ top-quark partner. A spin-1/2 top-quark partner with a mass between $300 \mathrm{GeV}$ and $480 \mathrm{GeV}$, decaying to a top quark and a neutral non-interacting particle lighter than $100 \mathrm{GeV}$, is excluded at $95 \%$ confidence level.

KEYwORDS: Hadron-Hadron Scattering 


\section{Contents}

1 Introduction 1

2 The ATLAS detector 2

3 Monte Carlo samples $\quad 3$

4 Physics object reconstruction $\quad 4$

5 Event selection $\quad 5$

$\begin{array}{llr}6 & \text { Background estimation } & 6\end{array}$

$\begin{array}{llr}7 & \text { Systematic uncertainties } & 8\end{array}$

8 Results 10

9 Conclusions 12

$\begin{array}{ll}\text { The ATLAS collaboration } & 19\end{array}$

\section{Introduction}

Partners of the top quark are an ingredient of several models addressing the hierarchy problem of the Standard Model (SM). In order to stabilize the Higgs boson mass against divergent quantum corrections, these new particles should have masses close to the electroweak symmetry breaking energy scale, and thus be accessible at the LHC. One of these models is Supersymmetry (SUSY) [1-9] which naturally resolves the hierarchy problem [10-13] by introducing supersymmetric partners of the known bosons and fermions. In the MSSM [14-18], an R-parity conserving minimal supersymmetric extension of the SM, the scalar partners of right-handed and left-handed quarks, $\tilde{q}_{R}$ and $\tilde{q}_{L}$, can mix to form two mass eigenstates. In this paper a search for a scalar top $\tilde{t}_{1}$ which decays into a top quark and the lightest neutralino $\tilde{\chi}_{1}^{0}$ is performed. In this model, the $\tilde{\chi}_{1}^{0}$ is a stable particle which would escape detection.

A top-quark fermionic partner $T$ which decays into a stable, neutral, weakly interacting particle $A_{0}$ also appears in other SM extensions, such as little Higgs models with $T$-parity conservation [19-21] or models of Universal Extra Dimensions (UED) with Kaluza-Klein parity [22]. The production cross section at the LHC is predicted to be approximately six times higher for fermionic $T$ [23] than for the $\tilde{t}_{1}$. Furthermore, scalar top and $T$ decay kinematic distributions differ due to helicity effects in the decay, yielding different experimental acceptances. 
Searches for these spin-1/2 heavy top-quark partners were performed by the CDF Collaboration in proton-antiproton collisions at $\sqrt{s}=1.96 \mathrm{TeV}$ [24], excluding at $95 \%$ confidence level (CL) top-quark partners with masses up to $400 \mathrm{GeV}$ for an $A_{0}$ mass lower than $70 \mathrm{GeV}$. A previous ATLAS analysis with $1.04 \mathrm{fb}^{-1}$ of proton-proton collisions at $\sqrt{s}=7 \mathrm{TeV}[25]$ excludes a $T$ with masses up to $420 \mathrm{GeV}$.

In this paper a search for the direct pair production of heavy top-quark partners is presented, where $\tilde{t}_{1} \rightarrow t \tilde{\chi}_{1}^{0}$ or $T \rightarrow t A_{0}$. The final state targeted by the analysis includes two top quarks and additional missing transverse momentum $\mathbf{p}_{\mathrm{T}}^{\text {miss }}$, with magnitude $E_{\mathrm{T}}^{\text {miss }}$, resulting mainly from the undetected $\tilde{\chi}_{1}^{0}$ or $A_{0}$. The present study addresses the two-lepton signature resulting from the leptonic decay of both the $W$ bosons from the top-quark decay. The neutrinos from the $W$ decays also contribute to the missing transverse momentum. Events with two electrons, two muons, or an electron-muon pair in the final state are selected by the analysis. To separate the signal from the large irreducible background from top-quark pair production, the $m_{\mathrm{T} 2}$ variable $[26,27]$ is used. It is defined as:

$$
m_{\mathrm{T} 2}\left(\mathbf{p}_{\mathrm{T}}^{\ell_{1}}, \mathbf{p}_{\mathrm{T}}^{\ell_{2}}, \mathbf{p}_{\mathrm{T}}^{\mathrm{miss}}\right)=\min _{\mathbf{q}_{\mathrm{T}}+\mathbf{r}_{\mathrm{T}}=\mathbf{p}_{\mathrm{T}}^{\text {miss }}}\left\{\max \left[m_{\mathrm{T}}\left(\mathbf{p}_{\mathrm{T}}^{\ell_{1}}, \mathbf{q}_{\mathrm{T}}\right), m_{\mathrm{T}}\left(\mathbf{p}_{\mathrm{T}}^{\ell_{2}}, \mathbf{r}_{\mathrm{T}}\right)\right]\right\}
$$

where $m_{\mathrm{T}}$ indicates the transverse mass, $\mathbf{p}_{\mathrm{T}}^{\ell_{1}}$ and $\mathbf{p}_{\mathrm{T}}^{\ell_{2}}$ are the transverse momenta of the two leptons, and $\mathbf{q}_{\mathrm{T}}$ and $\mathbf{r}_{\mathrm{T}}$ are vectors which satisfy $\mathbf{q}_{\mathrm{T}}+\mathbf{r}_{\mathrm{T}}=\mathbf{p}_{\mathrm{T}}^{\text {miss }}$. The minimization is performed over all the possible decompositions of $\mathbf{p}_{\mathrm{T}}^{\text {miss }}$. The distribution of this variable presents a sharp kinematic limit at the $W$ boson mass for $t \bar{t}$ production [28, 29], whereas for the signal topology it decreases slowly towards a higher mass value, due to the presence of the two additional invisible particles produced in association with the top-quark pair. The results are interpreted in the scalar top-neutralino mass plane as well as in a generic model producing a heavy spin- $1 / 2$ top-quark partner $T$ decaying into an invisible particle $A_{0}$ and a top quark.

This analysis is sensitive to masses of the top-quark partner in excess of about $200 \mathrm{GeV}$ and is thus complementary to a parallel ATLAS study reported in refs. [30, 31] optimized for scalar top masses near or below the top mass.

\section{The ATLAS detector}

The ATLAS detector [32] consists of inner tracking devices surrounded by a superconducting solenoid, electromagnetic and hadronic calorimeters and a muon spectrometer with a toroidal magnetic field. The inner detector, in combination with the axial $2 \mathrm{~T}$ field from the solenoid, provides precision tracking of charged particles for $|\eta|<2.5$, where the pseudorapidity $\eta$ is defined in terms of the angle $\theta$ with the beam pipe axis as $\eta=-\ln \tan (\theta / 2)$. It consists of a silicon pixel detector, a silicon strip detector and a straw tube tracker that also provides transition radiation measurements for electron identification. The calorimeter system covers the pseudorapidity range $|\eta|<4$.9. It is composed of sampling calorimeters with either liquid argon or scintillating tiles as the active media. The muon spectrometer has separate trigger and high-precision tracking chambers which provide muon trigger and measurement capabilities for $|\eta|<2.4$ and $|\eta|<2.7$ respectively. 


\begin{tabular}{|l|c|c|}
\hline Physics process & $\sigma \cdot \mathrm{BR}[\mathrm{pb}]$ & Perturbative order \\
\hline$Z / \gamma^{\star} \rightarrow \ell^{+} \ell^{-}$ & $1069 \pm 53$ & NNLO \\
$t \bar{t}$ & $167_{-18}^{+17}$ & NLO+NNLL \\
$W t$ & $15.7 \pm 1.2$ & NLO+NNLL \\
$t \bar{t} W$ & $0.168_{-0.037}^{+0.023}$ & NLO \\
$t \bar{t} Z$ & $0.130 \pm 0.019$ & NLO \\
$W W$ & $44.4 \pm 2.8$ & NLO \\
$W Z$ & $19.1 \pm 1.3$ & NLO \\
$Z Z$ & $6.2 \pm 0.3$ & NLO \\
\hline
\end{tabular}

Table 1. The most important SM background processes and their production cross sections, multiplied by the relevant branching ratios. The $\ell$ indicates all three types of leptons $(e, \mu, \tau)$ summed together. The $Z / \gamma^{\star}$ production cross section is given for events with a di-lepton invariant mass of at least $12 \mathrm{GeV}$.

\section{Monte Carlo samples}

Monte Carlo (MC) simulated event samples are used to aid in the description of the background and to model the SUSY and spin-1/2 heavy top-quark partner signals.

Top-quark pair and $W t$ production are simulated with MC@NLO [33, 34], interfaced with HERWIG [35] for the fragmentation and the hadronization processes, including JIMMY [36] for the underlying event. The top-quark mass is fixed at $172.5 \mathrm{GeV}$, and the next-to-leading-order (NLO) parton distribution function (PDF) set CTEQ10 [37] is used. Additional $\mathrm{MC}$ samples are used to estimate the event generator systematic uncertainties: two POWHeg [38] samples, one interfaced with HERWIG and the other with PYTHIA [39]; an ALPGEN [40] sample interfaced with HERWIG and JIMMY; two ACERMC [41] samples produced with variations to the PYTHIA parton shower parameters chosen such that the two samples produce additional radiation consistent with the experimental uncertainty in the data $[42,43]$.

Samples of $Z / \gamma^{\star}$ produced in association with light- and heavy-flavour jets are generated with ALPGEN using the PDF set CTEQ6.1 [44]. Samples of $t \bar{t} Z$ and $t \bar{t} W$ production are generated with MAdGRAPH [45] interfaced to PYTHIA. Diboson samples ( $W W, W Z$, $Z Z$ ) are generated with SHERPA [46]. Additional samples generated with ALPGEN and HERWIG are used for the evaluation of the event generator systematic uncertainties.

The background predictions are normalized to theoretical cross sections, including higher-order QCD corrections when available, and are compared to data in control regions populated by events produced by SM processes. Next-to-next-to-leading-order (NNLO) cross sections are used for inclusive $Z$ boson production [47, 48]. Approximate NLO+NNLL (next-to-next-to-leading-logarithms) cross sections are used in the normalization of the $t \bar{t}[49]$ and $W t$ [50] samples. NLO cross sections are used for the diboson samples [33, 51] and for the $t \bar{t} W$ and $t \bar{t} Z$ [52] samples. Production of $t \bar{t}$ in association with $b \bar{b}$ is normalized to leading order (LO) cross section [40]. Table 1 summarizes the production cross sections used in this analysis and their uncertainties. 
SM processes that generate jets which are misidentified as leptons, or where a lepton from a $b$-hadron or $c$-hadron decay is selected, collectively referred to as "fake" leptons in the following, are estimated from data as described in section 6 .

Scalar top signal samples are generated with HERWIG $++[53]$. The mixings in the scalar top and gaugino sector are chosen to be such that the lightest scalar top is mostly the partner $\tilde{t}_{\mathrm{R}}$ of the right-handed top quark, and the lightest neutralino is almost a pure bino. Under such conditions, the scalar top is expected to decay to the lightest neutralino and a top quark with a branching ratio close to $100 \%$, even if the decay mode to a chargino and a $b$ quark is kinematically allowed. The effects of helicity in the decay are correctly treated by HERWIG ++ . Spin-1/2 heavy top-quark partner signal samples are generated with MADGRAPH [45]. Signal cross sections are calculated to NLO in the strong coupling constant, including the resummation of soft gluon emission at next-to-leading-logarithmic accuracy (NLO+NLL) [54-56], as described in ref. [57].

The MC generator parameters have been tuned to ATLAS data $[58,59]$ and generated events have been processed through a detector simulation [60] based on GEANT4 [61]. Effects of multiple proton-proton interactions in the same bunch crossing (pile-up) are included, with the MC samples re-weighted so that the distribution of the average number of interactions per bunch crossing agrees with that in the data.

\section{Physics object reconstruction}

Proton-proton interaction vertex candidates are reconstructed using the Inner Detector tracks. The vertex with the highest scalar sum of the $p_{\mathrm{T}}$ of the associated tracks is defined as the primary vertex.

Jets are reconstructed from three-dimensional calorimeter energy clusters using the anti- $k_{t}$ jet algorithm $[62,63]$ with a radius parameter of 0.4 . The measured jet energy is corrected for inhomogeneities, and the non-compensating nature of the calorimeter with $p_{\mathrm{T}^{-}}$ and $\eta$-dependent correction factors [64]. Only jet candidates with $p_{\mathrm{T}}>20 \mathrm{GeV},|\eta|<2.5$ and a "jet vertex fraction" larger than 0.75 are retained. Based on tracking information, the jet vertex fraction quantifies the fraction of a jet's momentum that originates from the reconstructed primary vertex. The requirement on the jet vertex fraction rejects jets originating from additional proton-proton interactions occurring in the same bunch crossing. Events with any jet that fails the jet quality criteria designed to reject noise and non-collision backgrounds [64] are rejected.

Electron candidates are required to have $p_{\mathrm{T}}>20 \mathrm{GeV},|\eta|<2.47$ and to satisfy "medium" electromagnetic shower shape and track selection quality criteria [65]. These preselected electrons are then required to pass "tight" quality criteria [65] which places additional requirements on the ratio of calorimetric energy to track momentum, and on the fraction of hits in the straw tube tracker that pass a higher threshold for transition radiation. The electron candidates are then required to be isolated: the scalar sum of the $p_{\mathrm{T}}, \Sigma p_{\mathrm{T}}$, of inner detector tracks, not including the electron track, with $p_{\mathrm{T}}>1 \mathrm{GeV}$ within a cone in the $\eta-\phi$ plane of radius $\Delta R=\sqrt{\Delta \eta^{2}+\Delta \phi^{2}}=0.2$ around the electron candidate must be less than $10 \%$ of the electron $p_{\mathrm{T}}$. 
Muon candidates are reconstructed using either a full muon spectrometer track matched to an inner detector track, or a muon spectrometer segment matched to an extrapolated inner detector track [66]. They must be reconstructed with sufficient hits in the pixel, strip and straw tube detectors. They are required to have $p_{\mathrm{T}}>10 \mathrm{GeV},|\eta|<2.4$ and must have longitudinal and transverse impact parameters within $1 \mathrm{~mm}$ and $0.2 \mathrm{~mm}$ of the primary vertex, respectively. Such preselected candidates are then required to have $\Sigma p_{\mathrm{T}}<1.8 \mathrm{GeV}$, defined in analogy to the electron case.

Following the object reconstruction described above, overlaps between jet, electron and muon candidates are resolved. Any jet within $\Delta R=0.2$ of preselected electrons is discarded. Electrons or muons within $\Delta R=0.4$ of any remaining jet are then discarded to reject leptons from the decay of a $b$ - or $c$-hadron.

The $E_{\mathrm{T}}^{\text {miss }}$ is the magnitude of the vectorial sum of the $p_{\mathrm{T}}$ of the reconstructed jets (with $p_{\mathrm{T}}>20 \mathrm{GeV}$ and $|\eta|<4.5$ ) after overlap removal, preselected leptons and clusters of calorimeter cells not belonging to reconstructed physics objects [67].

A $b$-tagging algorithm exploiting both impact parameter and secondary vertex information [68] is used to identify jets containing a $b$-hadron decay. The chosen operating point has a $60 \%$ efficiency for tagging $b$-jets in a MC sample of $t \bar{t}$ events, with a mis-tag probability of less than $1 \%$ for jets from light quarks and gluons.

\section{Event selection}

This search uses proton-proton collisions recorded in 2011 at a centre-of-mass energy of $7 \mathrm{TeV}$. The data are selected with a three-level trigger system. Events are accepted if they pass either a single-electron trigger reaching a plateau efficiency of about $97 \%$ for electrons with $p_{\mathrm{T}}>25 \mathrm{GeV}$, or a single-muon or combined muon+jet trigger which reaches a plateau efficiency of about $75 \%(90 \%)$ in the barrel (end-caps) for events including muons with $p_{\mathrm{T}}>20 \mathrm{GeV}$ and jets with $p_{\mathrm{T}}>50 \mathrm{GeV}$. The combined muon+jet trigger is used for the data-taking periods with high instantaneous luminosity, because it is based on looser muon identification requirements than the single-muon trigger available for those periods, resulting in a higher plateau efficiency. Events are required to have a reconstructed primary vertex with five or more tracks consistent with the transverse beam spot position. Following beam, detector and data quality requirements, a total integrated luminosity of $(4.7 \pm 0.2) \mathrm{fb}^{-1}$ is used, measured as described in refs. [69, 70].

Two signal regions (SRs) are defined, one for different-flavour, and one for same-flavour leptons. For both SRs events are required to have exactly two opposite-sign (OS) leptons (electrons or muons) with an invariant mass larger than $20 \mathrm{GeV}$. At least one electron or muon must have a momentum in the trigger efficiency plateau regions described above. If the event contains a third preselected electron or muon, the event is rejected. At least two jets with $p_{\mathrm{T}}>25 \mathrm{GeV}$, and at least one of them with $p_{\mathrm{T}}>50 \mathrm{GeV}$, are required. This requirement suppresses $W W$ and $Z / \gamma^{\star}+$ jets backgrounds.

For the same-flavour SR, additional selections are imposed to suppress the $Z / \gamma^{\star}+$ jets, $W Z$ and $Z Z$ backgrounds, which represent a significant fraction of events with large $m_{\mathrm{T} 2}$. These events have large $E_{\mathrm{T}}^{\text {miss }}$, which for the $W Z$ process is generated by the leptonic decay 


\begin{tabular}{|l|c|c|c|c|c|}
\hline Top quark partner mass $[\mathrm{GeV}]$ & 200 & 300 & 400 & 500 & 600 \\
\hline$\tilde{t}_{1} \tilde{t}_{1}$ production & $0.02 \%$ & $7.7 \%$ & $22.0 \%$ & $35.6 \%$ & $43.0 \%$ \\
$T T$ production & - & $5.3 \%$ & $15.8 \%$ & $27.3 \%$ & $34.3 \%$ \\
\hline
\end{tabular}

Table 2. Efficiency of the $m_{\mathrm{T} 2}$ selection, calculated after all other selection requirements applied in the SR, for signal samples with different values of the mass of the scalar top or of the spin- $1 / 2$ heavy top-quark partner. The mass of the $\tilde{\chi}_{1}^{0}$ or $A_{0}$ is zero in each case. No signal sample with mass $m(T)=200 \mathrm{GeV}$ has been simulated.

of the $W$ boson, for the $Z Z$ process by the decay of one of the $Z$ bosons to neutrinos, and for $Z / \gamma^{\star}+$ jets by the tails in the jet energy resolution. The additional selections required in the same-flavour channel to suppress these backgrounds are that the invariant mass of the leptons must be outside the $71-111 \mathrm{GeV}$ range, and at least one of the jets must be tagged as a $b$-jet. After these selections the background is dominated by $t \bar{t}$.

Finally, for both SRs, signal candidate events are required to have a value of $m_{\mathrm{T} 2}$ larger than $120 \mathrm{GeV}$. This requirement suppresses the remaining $t \bar{t}$ and $W W$ backgrounds by several orders of magnitude and was chosen to optimize the coverage of the analysis in the $\tilde{t}_{1}-\tilde{\chi}_{1}^{0}$ and $T-A_{0}$ planes.

Before the $m_{\mathrm{T} 2}$ selection, $t \bar{t}$ production is by far the largest background. The efficiency of the $m_{\mathrm{T} 2}$ selection for $t \bar{t}$ events, calculated after all the other SR cuts, is $0.007 \%$. The efficiency of the $m_{\mathrm{T} 2}$ selection for scalar top and spin-1/2 heavy top-quark partner signal samples is given in table 2 for several values of the top-quark partner mass and for a massless $\tilde{\chi}_{1}^{0}$ or $A_{0}$. The efficiency is smallest when $\Delta m=m\left(\tilde{t}_{1}\right)-m\left(\tilde{\chi}_{1}^{0}\right)$ or $m(T)-m\left(A_{0}\right)$ is close to the top quark mass, because the kinematics of the signal are then similar to those of $t \bar{t}$ background, and it increases with increasing $\Delta m$. For equal masses, the spin$1 / 2$ top-quark partner signals have a slightly lower efficiency than the scalar top signals, due to helicity effects in the decay.

\section{Background estimation}

The dominant SM background contributions to the SRs are top-quark pair and $Z / \gamma^{\star}+$ jets production. They are evaluated by defining a control region $(\mathrm{CR})$ populated mostly by the targeted background, and using $\mathrm{MC}$ simulation to extrapolate from the rate measured in the CR to the expected background yield in the SR:

$$
N(\mathrm{SR})=\left(N^{\text {Data }}(\mathrm{CR})-N_{\text {others }}(\mathrm{CR})\right) \frac{N^{\mathrm{MC}}(\mathrm{SR})}{N^{\mathrm{MC}}(\mathrm{CR})}
$$

where $N^{\text {Data }}(\mathrm{CR})$ is the number of data events observed in the $\mathrm{CR}, N^{\mathrm{MC}}(\mathrm{CR})$ and $N^{\mathrm{MC}}(\mathrm{SR})$ are the number of events of the targeted background expected from MC simulation in the CR and SR respectively, and the term $N_{\text {others }}(\mathrm{CR})$ is the contribution from the other background sources in the $\mathrm{CR}$ which is estimated from MC simulation or additional control samples in data. The ratio of the number of MC events in the SR to the number of MC 


\begin{tabular}{|l|c|c|}
\hline & $t \bar{t}$ CR & $t \bar{t}$ CR \\
Process & DF & SF \\
\hline$t \bar{t}$ & $68 \pm 11$ & $39 \pm 11$ \\
$t \bar{t} W+t \bar{t} Z$ & $0.37 \pm 0.07$ & $0.20 \pm 0.05$ \\
$W t$ & $2.7 \pm 1.0$ & $1.8 \pm 0.6$ \\
$Z / \gamma^{\star}+$ jets & - & $3.5 \pm 1.4$ \\
Fake leptons & $0.4 \pm 0.3$ & $0.5 \pm 1.6$ \\
Diboson & $0.49 \pm 0.14$ & $0.10 \pm 0.05$ \\
\hline Total non- $t \bar{t}$ & $4.0 \pm 1.5$ & $6.1 \pm 3.7$ \\
Total expected & $72 \pm 11$ & $45 \pm 12$ \\
\hline Data & 79 & 53 \\
\hline
\end{tabular}

Table 3. Expected background composition and comparison of the predicted total SM event yield to the observed number of events in the top-quark control regions described in the text for the same-flavour (SF) and different-flavour (DF) selections. The expected $Z / \gamma^{\star}+$ jets rate in the DF channel is negligible. The quoted uncertainties include the systematic uncertainties described in section 7 .

events in the CR for a given background source is referred to as the "transfer factor" in the following.

The $t \bar{t} \mathrm{CR}$ is defined akin to the SR, except for $m_{\mathrm{T} 2}$, which is required to be between $85 \mathrm{GeV}$ and $100 \mathrm{GeV}$. The expected background composition of the $t \bar{t} \mathrm{CR}$ is given in table 3. The contamination due to fake leptons is evaluated from data with the technique described below, while all the other processes are obtained from the MC prediction. The $t \bar{t}$ background is expected to account for $86 \%$ and $94 \%$ of the SM rate in the same-flavour and different-flavour CRs, respectively. The number of observed events is in good agreement with the expected event yields.

The systematic uncertainties on the modelling of the $t \bar{t}$ background transfer factor due to the choice of the MC generator are assessed by comparing the baseline sample simulated with MC@NLO with the alternative samples described in section 3.

The background from $Z / \gamma^{\star}+$ jets is relevant for the same-flavour selection in the case of the decay channels $Z \rightarrow e e$ or $\mu \mu$. For $Z \rightarrow \tau \tau$ decays, which would contribute both to the same-flavour and the different-flavour samples, the $m_{\mathrm{T} 2}$ distribution falls very steeply, and the number of expected events for $m_{\mathrm{T} 2}$ in excess of $80 \mathrm{GeV}$ is negligible.

The CR for $Z / \gamma^{\star}+$ jets is defined with the same selections as for the SR, except for the $Z$ boson veto selection which is reversed. The observed number of events in this CR is 11, compared to 7.6 \pm 1.1 expected, of which $7.0 \pm 1.1$ are from $Z$ boson production, where the quoted uncertainties include the systematics discussed in the next section. The transfer factor between $\mathrm{CR}$ and SR is evaluated with $Z / \gamma^{\star}+$ jet MC samples to which all the selections of the same-flavour analysis except the $b$-tagging requirement are applied. Detailed checks have been performed in order to verify that this transfer factor, which relates the number of events inside the $Z$ boson peak to the number of events outside, is 
stable with respect to the $m_{\mathrm{T} 2}$ and $b$-tag requirements. The method is validated using an auxiliary CR dominated by $Z / \gamma^{\star}+$ jets events, defined in the same way as the SR except the $b$-jet requirement is removed. The number of predicted background events is $7.5 \pm 1.3$ (of which $7.2 \pm 1.3$ from $Z / \gamma^{\star}+$ jets) while the observed number is 10 . The quoted uncertainty on the prediction is only statistical.

Additional SM processes yielding two isolated leptons and $E_{\mathrm{T}}^{\mathrm{miss}}(W t, W W, W Z, Z Z$, $t \bar{t} W, t \bar{t} Z)$ are estimated from the MC simulation. The contribution from diboson processes, particularly $W W$ production, is about a quarter of the total background in the differentflavour signal region. The high- $m_{\mathrm{T} 2}$ population in $W W$ production is dominated by events in which a strongly off-shell $W$ is produced. The $18 \%$ relative difference in the number of events with $m_{\mathrm{T} 2}>120 \mathrm{GeV}$ between SHERPA and HERWIG before jet selection is taken as a systematic uncertainty on the simulation of the $m_{\mathrm{T} 2}$ distribution. The $45 \%$ relative difference between SHERPA and ALPGEN in the efficiency of the jet selections integrated over the whole $m_{\mathrm{T} 2}$ range is taken as a systematic uncertainty on the $W W j j$ cross section.

The fake lepton background consists of semi-leptonic $t \bar{t}, s$-channel and $t$-channel single top, $W+$ jets and light- and heavy-flavour jet production. The contribution from this background is small (less than 10\% of the total background). It is estimated from data with a method similar to that described in refs. [71, 72]. Two types of lepton identification criteria are defined for this evaluation: "tight", corresponding to the full set of identification criteria described above, and "loose" corresponding to preselected electrons and muons. The method counts the number of observed events containing loose-loose, loose-tight, tightloose and tight-tight lepton pairs in the SR. The probability for real leptons passing the loose selection criteria to also pass the tight selection is measured using a $Z \rightarrow \ell \ell$ sample. The equivalent probability for fake leptons is measured from multijet-enriched control samples. From these probabilities the number of events containing a contribution from one or two fake leptons is calculated.

The procedure described above is used to estimate the fake lepton background with looser selections and extrapolated to the signal region. A systematics uncertainty is assigned to the extrapolation procedure by comparing the direct and extrapolated background estimate in various control regions.

\section{Systematic uncertainties}

Various systematic uncertainties affecting the predicted background rates in the signal regions are considered. Such uncertainties are either used directly in the evaluation of the predicted background in the SR (for diboson, $W t, t \bar{t} W$ and $t \bar{t} Z$ production), or to compute the uncertainty on the transfer factor and propagate it to the predicted event yields in the SR (for $t \bar{t}, Z / \gamma^{\star}+$ jets).

The following experimental systematic uncertainties were found to be non-negligible:

Jet energy scale and resolution. The uncertainty on the jet energy scale (JES), derived using single particle response and test beam data, varies as a function of the jet $p_{\mathrm{T}}$ and pseudorapidity [64]. Additional systematic uncertainties arise from the dependence of the jet response on the number of interactions per bunch crossing and on 
the jet flavour. The total jet energy scale uncertainty at $p_{\mathrm{T}}=50 \mathrm{GeV}$ in the central detector region is about $5 \%$ [64]. The components of the jet energy scale uncertainty are varied by $\pm 1 \sigma$ in the $\mathrm{MC}$ simulation in order to obtain the resulting uncertainty in the event yield. Uncertainties related to the jet energy resolution (JER) are obtained with an in situ measurement of the jet response asymmetry in dijet events [73]. Their impact on the event yield is estimated by applying an additional smearing to the jet transverse momenta. The JES and JER variations applied to the jet momenta are propagated to the $E_{\mathrm{T}}^{\text {miss }}$. The JES and JER relative uncertainties on the same-flavour and different-flavour signal region event yield amount to $16 \%$ and $22 \%$, respectively.

Calorimeter cluster energy scale and pile-up modelling. The uncertainties related to the contribution to $E_{\mathrm{T}}^{\text {miss }}$ from the energy in the calorimeter cells not associated to electrons, muons or jets, and also from low momentum $\left(7 \mathrm{GeV}<p_{\mathrm{T}}<25 \mathrm{GeV}\right)$ jets, as well as the uncertainty due to the modelling of pile-up have been evaluated to amount to $6 \%(25 \%)$ of the same-flavor (different-flavor) event yield. The fractional uncertainty is smaller in the same-flavour channel because it has a very small impact (2\%) on the estimation of the $Z / \gamma^{\star}+$ jets background, which is by far the largest contribution to the same-flavour channel.

$\boldsymbol{b}$-tagging efficiency and mis-tagging uncertainties. This uncertainty is evaluated by varying the $b$-tagging efficiency and mis-tagging rates within the uncertainties measured in situ [68]. Since the different-flavour selection does not make use of $b$ tagging, this uncertainty only affects the same-flavour channel and is relatively small (about $1 \%$ of the total event yield).

Fake-lepton background uncertainties: an uncertainty of $33 \%(25 \%)$ is assigned to the fake background in the same-flavour (different-flavour) channel from the comparison of results from different CRs, with an additional $30 \%$ is taken as the systematic uncertainty due to the projection of events into the SR.

Other significant sources of uncertainty are the normalization uncertainties for processes estimated from MC simulation only, the theoretical uncertainties discussed in section 6, the limited number of data events in the CRs, the limited number of $\mathrm{MC}$ events, and the integrated luminosity.

A summary of the uncertainties on the total expected background in the two channels is given in table 4. The row labelled "statistics" includes the effects of the limited number of data events in the CRs and the limited number of MC events. The theoretical uncertainties include the cross section, MC generator, and initial- and final-state radiation uncertainties. They are smaller for the same-flavor channel because the theoretical uncertainty on the $Z / \gamma^{\star}+$ jets is relatively small (about $10 \%$ ). In the opposite-flavour channel the dominant backgrounds are the top pair production, which is affected by an uncertainty of about $100 \%$ (due to the description of the high $m_{\mathrm{T} 2}$ tail in simulation), and the diboson process whose cross section has an uncertainty of about $50 \%$, due mostly to the poor prediction of the production in association with two (or more) jets. 


\begin{tabular}{|l|c|c|}
\hline Channel & SF & DF \\
\hline Total event yield & 1.58 & 0.94 \\
\hline JES + JER & $16 \%$ & $22 \%$ \\
$b$-tagging & $1 \%$ & - \\
$E_{\mathrm{T}}^{\text {miss }}$ and pile-up modeling & $6 \%$ & $25 \%$ \\
Luminosity & $1 \%$ & $2 \%$ \\
Theory & $14 \%$ & $48 \%$ \\
Statistics & $\pm_{26}^{29} \%$ & $20 \%$ \\
Fake-lepton uncertainties & $\pm_{0}^{8} \%$ & $\pm_{0}^{9} \%$ \\
\hline Total uncertainty & $\pm_{37}^{40} \%$ & $64 \%$ \\
\hline
\end{tabular}

Table 4. Total expected background yield and uncertainties in the same-flavour (SF) and differentflavour (DF) signal regions. Where the uncertainty is not symmetric, the upwards and downwards values are given.

For the limit calculation, the uncertainty on the expected signal yield is also needed. The JES, JER, calorimeter energy scale and event pileup, and $b$-tagging uncertainties discussed above have been taken into account. The typical total uncertainty from these sources varies between $4 \%$ and $12 \%$ for the DF channel and between $7 \%$ and $22 \%$ for the SF channel, with comparable contributions from the JES, the calorimeter energy scale and pileup, and (for SF) the $b$-tagging.uncertainties.

The uncertainty on the signal cross sections is calculated with an envelope of cross section predictions which is defined using the $68 \%$ confidence level (CL) ranges of the CTEQ [74] (including the $\alpha_{S}$ uncertainty) and MSTW [75] PDF sets, together with variations of the factorization and renormalization scales by factors of two or one half. The nominal cross section value is taken to be the midpoint of the envelope and the uncertainty assigned is half the full width of the envelope, following the PDF4LHC recommendations [76] and using the procedure described in ref. [57]. The typical cross section uncertainty is $12 \%$ for the spin- $1 / 2$ top-quark partner signal and $15 \%$ for the scalar top signal.

\section{Results}

Figure 1 shows the distributions of the $m_{\mathrm{T} 2}$ variable for same-flavour and different-flavour events after all selection criteria are applied except the selection on $m_{\mathrm{T} 2}$ itself. For illustration, the distributions for two signal hypotheses are also shown. The data agree with the SM background expectation within uncertainties.

Table 5 shows the expected number of events in the SR for each background source and the observed number of events. No excess of events in data is observed, and limits at $95 \% \mathrm{CL}$ are derived on the visible cross section $\sigma_{\text {vis }}=\sigma \times \epsilon \times \mathcal{A}$, where $\sigma$ is the total production cross section for the non-SM signal, $\mathcal{A}$ is the acceptance defined by the fraction of events passing the geometric and kinematic selections at particle level, and $\epsilon$ is the detector reconstruction, identification and trigger efficiency. Limits are set using 

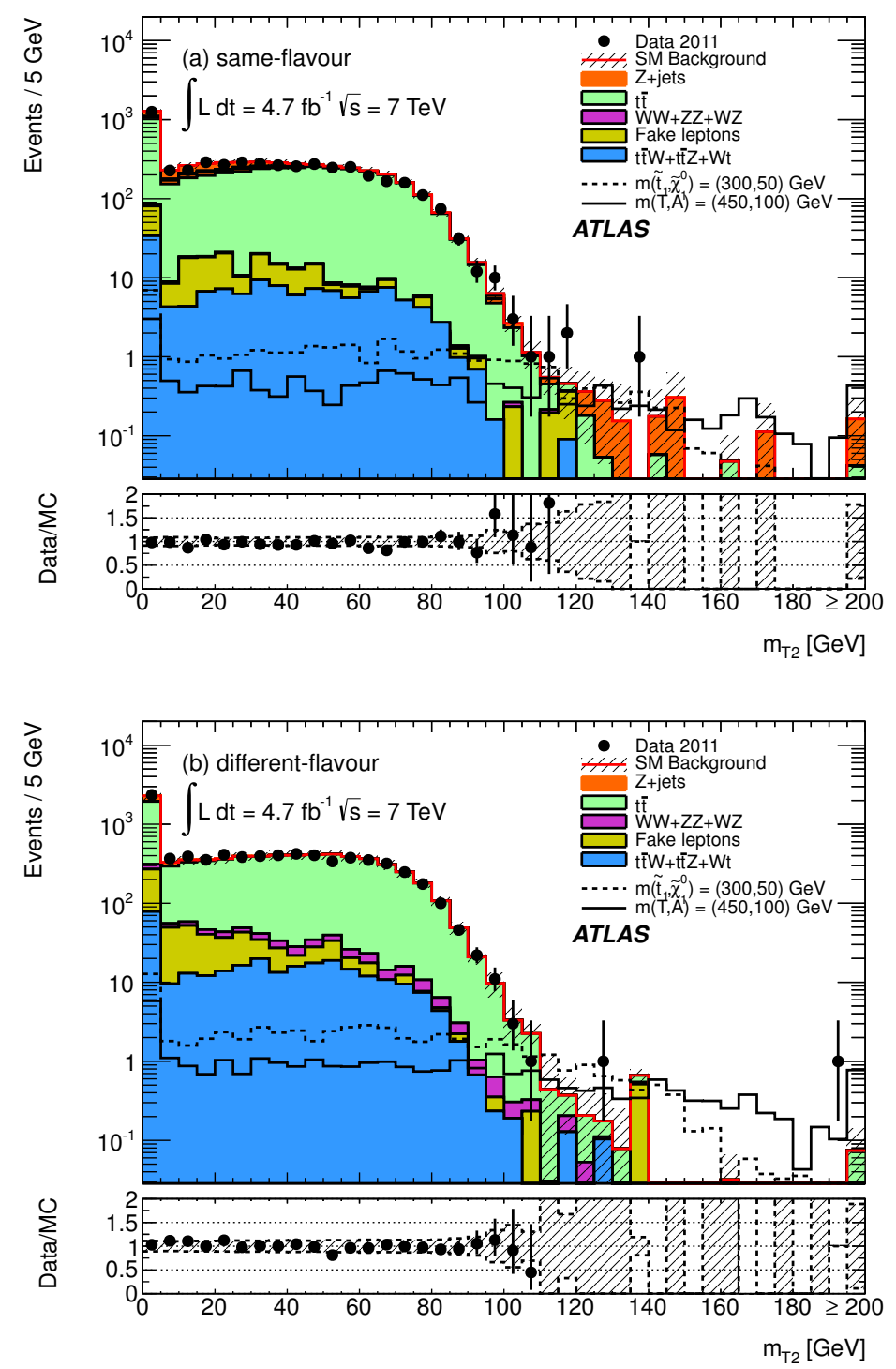

Figure 1. Distribution of $m_{\mathrm{T} 2}$ for events passing all the signal candidate selection requirements, except that on $m_{\mathrm{T} 2}$, for (a) same-flavour and (b) different-flavour events. The contributions from all SM backgrounds are shown; the bands represent the total uncertainties. The components labelled "fake lepton" are estimated from data as described in the text; the other backgrounds are estimated from MC simulation with normalizations measured in control regions described in section 6 for $t \bar{t}$ and $Z / \gamma^{\star}+$ jets . The distributions of the signal expected for two models considered in this paper are also shown: the dashed line corresponds to signal with a $300 \mathrm{GeV}$ scalar top and a $50 \mathrm{GeV}$ neutralino, while the solid line corresponds to a signal with a $450 \mathrm{GeV}$ spin- $1 / 2$ top quark partner $T$ and a $100 \mathrm{GeV} A_{0}$ particle.

the CLs likelihood ratio prescription as described in ref. [77]. Systematic uncertainties are included in the likelihood function as nuisance parameters with a gaussian probability density function. The limits are listed in table 5 . 


\begin{tabular}{|l|c|c|}
\hline & SF & DF \\
\hline$Z / \gamma^{\star}+$ jets & $1.2 \pm 0.5$ & - \\
$\left(Z / \gamma^{\star}+\right.$ jets scale factor $)$ & $(1.27)$ & $0.4 \pm 0.3$ \\
\hline$t \bar{t}$ & $0.23 \pm 0.23$ & $(1.10)$ \\
$(t \bar{t}$ scale factor $)$ & $(1.21)$ & $0.19 \pm 0.12$ \\
\hline$t \bar{t} W+t \bar{t} Z$ & $0.11 \pm 0.07$ & $0.19 \pm 0.18$ \\
$W W$ & $0.01_{-0.01}^{+0.02}$ & $0.03 \pm 0.03$ \\
$W Z+Z Z$ & $0.05 \pm 0.05$ & $0.10_{-0.10}^{+0.18}$ \\
$W t$ & $0.00_{-0.00}^{+0.17}$ & $0.00_{-0.00}^{+0.09}$ \\
Fake leptons & $0.00_{-0.00}^{+0.14}$ & $0.9 \pm 0.6$ \\
\hline Total SM & $1.6 \pm 0.6$ & 2 \\
\hline Observed & 1 & $3.7 \pm 0.6($ th. $) \pm 0.3($ exp. $)$ \\
\hline$m_{\tilde{t}_{1}}=300 \mathrm{GeV}, m_{\tilde{\chi}_{1}^{0}}=50 \mathrm{GeV}$ & $2.2 \pm 0.3($ th. $) \pm 0.2(\exp )$. & 1.08 \\
$m_{T}=450 \mathrm{GeV}, m_{A_{0}}=100 \mathrm{GeV}$ & $3.1 \pm 0.4($ th. $) \pm 0.3(\exp )$. & $5.8 \pm 0.7($ th. $) \pm 0.5($ exp. $)$ \\
\hline $95 \%$ CL limit on $\sigma_{\text {vis }}^{\text {obs }}[\mathrm{fb}]$ & 0.86 & 0.79 \\
$95 \%$ CL limit on $\sigma_{\text {vis }}^{\text {exp }}[\mathrm{fb}]$ & 0.89 & \\
\hline
\end{tabular}

Table 5. Number of expected SM background events and number of observed events for the sameflavour (SF) and different-flavour (DF) signal regions. The quoted errors are the total uncertainties on the expected rates. For $Z / \gamma^{\star}+$ jets and $t \bar{t}$ the ratio between the estimate based on the control region and the MC prediction (scale factor) is also reported. Dashes indicate negligible background expectations. The expected yield for two signal models is also shown, with the associated theoretical and experimental uncertainties. Observed and expected upper limits at $95 \%$ confidence level on $\sigma_{\mathrm{vis}}=\sigma \times \epsilon \times \mathcal{A}$ are also shown.

The results obtained are used to derive limits on the mass of a pair-produced heavy top-quark partner decaying into a top quark and a weakly interacting particle with $100 \%$ branching ratio. The limits are derived in the plane defined by the masses of the two particles for two scenarios: a model with a scalar top $\tilde{t}_{1}$ and a spin- $-1 / 2$ neutralino $\tilde{\chi}_{1}^{0}$ and one with a spin- $1 / 2$ top-quark partner $T$ and a scalar boson $A_{0}$.

In both scenarios, the limits are derived after combining the same-flavour and differentflavour channels. Uncertainties on the detector response, cross section, luminosity and MC statistics are taken into account. The limits are shown in figure 2 for the scalar top and spin-1/2 top-quark partner models. Using a signal cross section one standard deviation below the central value, a spin-1/2 top-quark partner $\mathrm{T}$ with a mass between $300 \mathrm{GeV}$ and $480 \mathrm{GeV}$ (if the $A_{0}$ mass is lower than $100 \mathrm{GeV}$ ) is excluded at $95 \% \mathrm{CL}$. The region of the mass plane which is excluded is smaller for scalar top production, because of the lower production cross section. A scalar top of mass close to $300 \mathrm{GeV}$ and a nearly massless neutralino is excluded at $95 \% \mathrm{CL}$.

\section{Conclusions}

A search for a heavy partner of the top quark, which decays into a top quark and an invisible particle, has been performed using $4.7 \mathrm{fb}^{-1}$ of pp collision data at $\sqrt{s}=7 \mathrm{TeV}$ 

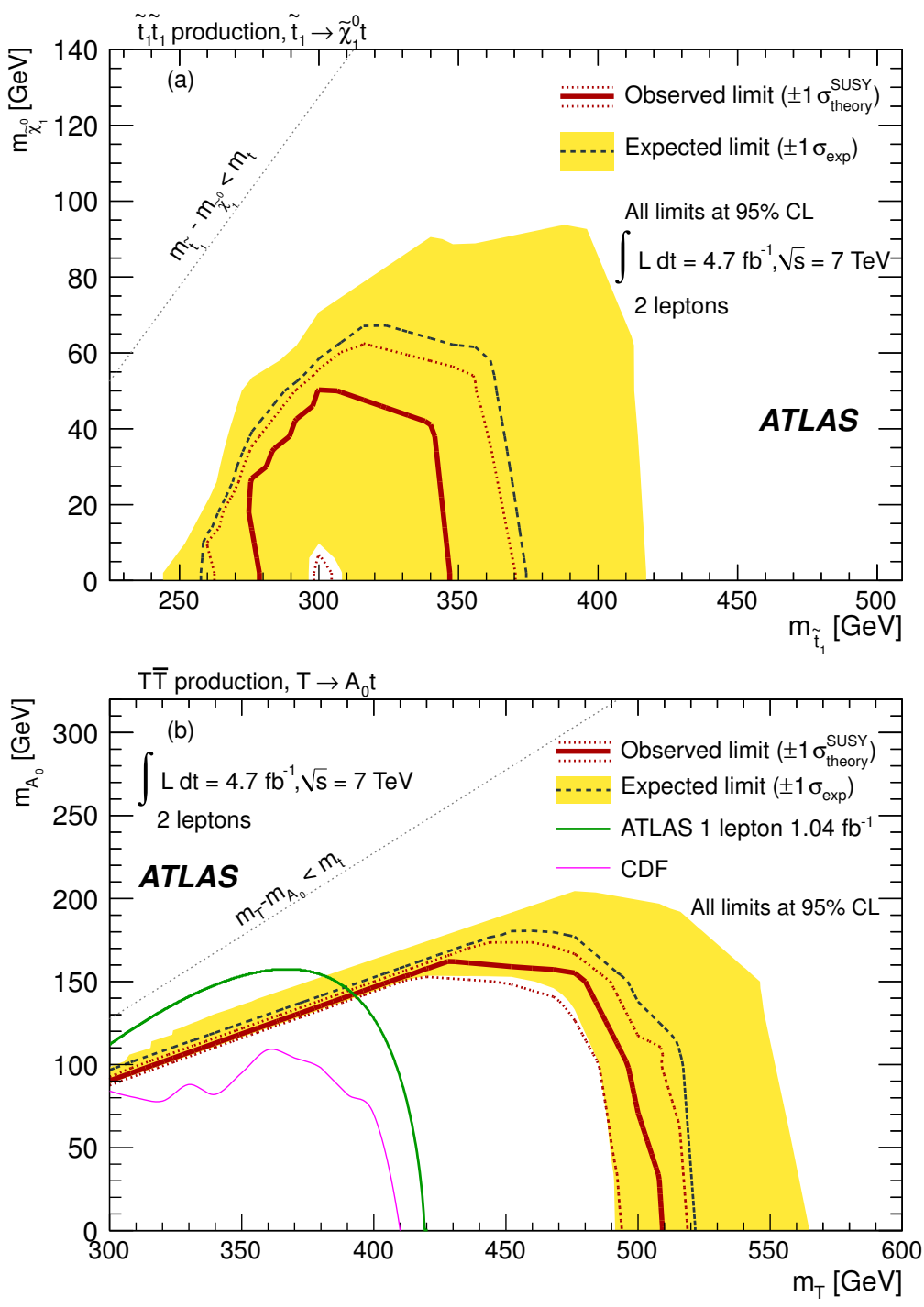

Figure 2. Expected and observed $95 \%$ CL limits (a) in the $\tilde{t}_{1} \rightarrow t \tilde{\chi}_{1}^{0}$ model as a function of the scalar top and neutralino masses, and (b) in the $T \rightarrow t A_{0}$ model as a function of the spin-1/2 topquark partner $T$ and $A_{0}$ masses. The dashed line and the shaded band are the expected limit and its $\pm 1 \sigma$ uncertainty, respectively. The thick solid line is the observed limit for the central value of the signal cross section. The expected and observed limits do not include the effect of the theoretical uncertainties on the signal cross section. The dotted lines show the effect on the observed limit of varying the signal cross section by $\pm 1 \sigma$ of the theoretical uncertainty. The curve labelled "ATLAS 1 lepton $1.04 \mathrm{fb}^{-1}$ " is the previous ATLAS limit from ref. [25] using the one lepton channel while the curve labelled "CDF" is from ref. [24].

produced by the LHC and taken by the ATLAS detector. The number of observed events has been found to be consistent with the Standard Model expectation.

Limits have been derived on a spin- $1 / 2$ heavy top-quark partner decaying to a top quark and a heavy neutral particle. A spin-1/2 top-quark partner mass between $300 \mathrm{GeV}$ and $480 \mathrm{GeV}$ is excluded at 95\% CL for a heavy neutral particle mass below $100 \mathrm{GeV}$. 
This result extends the previously published limits in this scenario [25]. A supersymmetric scalar top $\tilde{t}_{1}$ with a mass of $300 \mathrm{GeV}$ decaying to a top quark and a massless neutralino is also excluded at $95 \%$ CL.

The present result complements those from other ATLAS direct scalar top pair production searches $[30,31,78,79]$ addressing different signatures with either both scalar top decaying to a chargino and a $b$ quark $[30,31]$ or with both scalar top decaying to a neutralino and a top quark and the subsequent top quark decays yielding zero or one lepton in the final state $[78,79]$.

\section{Acknowledgments}

We thank CERN for the very successful operation of the LHC, as well as the support staff from our institutions without whom ATLAS could not be operated efficiently.

We acknowledge the support of ANPCyT, Argentina; YerPhI, Armenia; ARC, Australia; BMWF and FWF, Austria; ANAS, Azerbaijan; SSTC, Belarus; CNPq and FAPESP, Brazil; NSERC, NRC and CFI, Canada; CERN; CONICYT, Chile; CAS, MOST and NSFC, China; COLCIENCIAS, Colombia; MSMT CR, MPO CR and VSC CR, Czech Republic; DNRF, DNSRC and Lundbeck Foundation, Denmark; EPLANET and ERC, European Union; IN2P3-CNRS, CEA-DSM/IRFU, France; GNSF, Georgia; BMBF, DFG, HGF, MPG and AvH Foundation, Germany; GSRT, Greece; ISF, MINERVA, GIF, DIP and Benoziyo Center, Israel; INFN, Italy; MEXT and JSPS, Japan; CNRST, Morocco; FOM and NWO, Netherlands; RCN, Norway; MNiSW, Poland; GRICES and FCT, Portugal; MERYS (MECTS), Romania; MES of Russia and ROSATOM, Russian Federation; JINR; MSTD, Serbia; MSSR, Slovakia; ARRS and MVZT, Slovenia; DST/NRF, South Africa; MICINN, Spain; SRC and Wallenberg Foundation, Sweden; SER, SNSF and Cantons of Bern and Geneva, Switzerland; NSC, Taiwan; TAEK, Turkey; STFC, the Royal Society and Leverhulme Trust, United Kingdom; DOE and NSF, United States of America.

The crucial computing support from all WLCG partners is acknowledged gratefully, in particular from CERN and the ATLAS Tier-1 facilities at TRIUMF (Canada), NDGF (Denmark, Norway, Sweden), CC-IN2P3 (France), KIT/GridKA (Germany), INFN-CNAF (Italy), NL-T1 (Netherlands), PIC (Spain), ASGC (Taiwan), RAL (U.K.) and BNL (U.S.A.) and in the Tier-2 facilities worldwide.

Open Access. This article is distributed under the terms of the Creative Commons Attribution License which permits any use, distribution and reproduction in any medium, provided the original author(s) and source are credited.

\section{References}

[1] H. Miyazawa, Baryon Number Changing Currents, Prog. Theor. Phys. 36 (1966) 1266.

[2] P. Ramond, Dual Theory for Free Fermions, Phys. Rev. D 3 (1971) 2415 [InSPIRE].

[3] Y. Golfand and E. Likhtman, Extension of the Algebra of Poincaré Group Generators and Violation of $p$ Invariance, JETP Lett. 13 (1971) 323 [INSPIRE]. 
[4] A. Neveu and J. Schwarz, Factorizable dual model of pions, Nucl. Phys. B 31 (1971) 86 [INSPIRE].

[5] A. Neveu and J. Schwarz, Quark Model of Dual Pions, Phys. Rev. D 4 (1971) 1109 [inSPIRE].

[6] J.-L. Gervais and B. Sakita, Field theory interpretation of supergauges in dual models, Nucl. Phys. B 34 (1971) 632 [INSPIRE].

[7] D. Volkov and V. Akulov, Is the Neutrino a Goldstone Particle?, Phys. Lett. B 46 (1973) 109 [INSPIRE].

[8] J. Wess and B. Zumino, A Lagrangian Model Invariant Under Supergauge Transformations, Phys. Lett. B 49 (1974) 52 [INSPIRE].

[9] J. Wess and B. Zumino, Supergauge Transformations in Four-Dimensions, Nucl. Phys. B 70 (1974) 39 [INSPIRE].

[10] S. Weinberg, Implications of Dynamical Symmetry Breaking, Phys. Rev. D 13 (1976) 974 [INSPIRE].

[11] E. Gildener, Gauge Symmetry Hierarchies, Phys. Rev. D 14 (1976) 1667 [InSPIRE].

[12] S. Weinberg, Implications of Dynamical Symmetry Breaking: An Addendum, Phys. Rev. D 19 (1979) 1277 [INSPIRE].

[13] L. Susskind, Dynamics of Spontaneous Symmetry Breaking in the Weinberg-Salam Theory, Phys. Rev. D 20 (1979) 2619 [INSPIRE].

[14] P. Fayet, Supersymmetry and Weak, Electromagnetic and Strong Interactions, Phys. Lett. B 64 (1976) 159 [INSPIRE].

[15] P. Fayet, Spontaneously Broken Supersymmetric Theories of Weak, Electromagnetic and Strong Interactions, Phys. Lett. B 69 (1977) 489 [INSPIRE].

[16] G.R. Farrar and P. Fayet, Phenomenology of the Production, Decay and Detection of New Hadronic States Associated with Supersymmetry, Phys. Lett. B 76 (1978) 575 [INSPIRE].

[17] P. Fayet, Relations Between the Masses of the Superpartners of Leptons and Quarks, the Goldstino Couplings and the Neutral Currents, Phys. Lett. B 84 (1979) 416 [INSPIRE].

[18] S. Dimopoulos and H. Georgi, Softly Broken Supersymmetry and SU(5), Nucl. Phys. B 193 (1981) 150 [INSPIRE].

[19] H.-C. Cheng and I. Low, TeV symmetry and the little hierarchy problem, JHEP 09 (2003) 051 [hep-ph/0308199] [INSPIRE].

[20] H.-C. Cheng and I. Low, Little hierarchy, little Higgses and a little symmetry, JHEP 08 (2004) 061 [hep-ph/0405243] [INSPIRE].

[21] H.-C. Cheng, I. Low and L.-T. Wang, Top partners in little Higgs theories with T-parity, Phys. Rev. D 74 (2006) 055001 [hep-ph/0510225] [INSPIRE].

[22] T. Appelquist, H.-C. Cheng and B.A. Dobrescu, Bounds on universal extra dimensions, Phys. Rev. D 64 (2001) 035002 [hep-ph/0012100] [INSPIRE].

[23] J. Alwall, J.L. Feng, J. Kumar and S. Su, Dark Matter-Motivated Searches for Exotic 4 th Generation Quarks in Tevatron and Early LHC Data, Phys. Rev. D 81 (2010) 114027 [arXiv: 1002.3366] [INSPIRE].

[24] CDF collaboration, T. Aaltonen et al., Search for New T' Particles in Final States with Large Jet Multiplicities and Missing Transverse Energy in p $\bar{p}$ Collisions at $\sqrt{s}=1.96 \mathrm{TeV}$, Phys. Rev. Lett. 107 (2011) 191803 [arXiv:1107.3574] [InSPIRE]. 
[25] ATLAS collaboration, G. Aad et al., Search for New Phenomena in ttbar Events With Large Missing Transverse Momentum in Proton-Proton Collisions at $\sqrt{s}=7$ TeV with the ATLAS Detector, Phys. Rev. Lett. 108 (2012) 041805 [arXiv:1109.4725] [INSPIRE].

[26] C. Lester and D. Summers, Measuring masses of semiinvisibly decaying particles pair produced at hadron colliders, Phys. Lett. B 463 (1999) 99 [hep-ph/9906349] [INSPIRE].

[27] A. Barr, C. Lester and P. Stephens, $m_{T 2}$ : The Truth behind the glamour, J. Phys. G 29 (2003) 2343 [hep-ph/0304226] [INSPIRE].

[28] W.S. Cho, K. Choi, Y.G. Kim and C.B. Park, Measuring superparticle masses at hadron collider using the transverse mass kink, JHEP 02 (2008) 035 [arXiv:0711.4526] [INSPIRE].

[29] M. Burns, K. Kong, K.T. Matchev and M. Park, Using Subsystem $M_{T 2}$ for Complete Mass Determinations in Decay Chains with Missing Energy at Hadron Colliders, JHEP 03 (2009) 143 [arXiv:0810.5576] [INSPIRE].

[30] ATLAS collaboration, G. Aad et al., Search for light top squark pair production in final states with leptons and b-jets with the ATLAS detector in $\sqrt{s}=7$ TeV proton-proton collisions, arXiv:1209.2102 [INSPIRE].

[31] ATLAS collaboration, G. Aad et al., Search for light scalar top quark pair production in final states with two leptons with the ATLAS detector in $\sqrt{s}=7 \mathrm{TeV}$ proton-proton collisions, arXiv:1208.4305 [INSPIRE].

[32] ATLAS collaboration, G. Aad et al., The ATLAS Experiment at the CERN Large Hadron Collider, 2008 JINST 3 S08003 [INSPIRE].

[33] S. Frixione and B.R. Webber, Matching NLO QCD computations and parton shower simulations, JHEP 06 (2002) 029 [hep-ph/0204244] [INSPIRE].

[34] S. Frixione, E. Laenen, P. Motylinski and B.R. Webber, Single-top production in MC@NLO, JHEP 03 (2006) 092 [hep-ph/0512250] [INSPIRE].

[35] G. Corcella et al., HERWIG 6: An Event generator for hadron emission reactions with interfering gluons (including supersymmetric processes), JHEP 01 (2001) 010 [hep-ph/0011363] [INSPIRE].

[36] J. Butterworth, J.R. Forshaw and M. Seymour, Multiparton interactions in photoproduction at HERA, Z. Phys. C 72 (1996) 637 [hep-ph/9601371] [INSPIRE].

[37] H.-L. Lai et al., New parton distributions for collider physics, Phys. Rev. D 82 (2010) 074024 [arXiv: 1007.2241] [INSPIRE].

[38] S. Frixione, P. Nason and C. Oleari, Matching NLO QCD computations with Parton Shower simulations: the POWHEG method, JHEP 11 (2007) 070 [arXiv:0709.2092] [INSPIRE].

[39] T. Sjöstrand, S. Mrenna and P.Z. Skands, PYTHIA 6.4 Physics and Manual, JHEP 05 (2006) 026 [hep-ph/0603175] [INSPIRE].

[40] M.L. Mangano, M. Moretti, F. Piccinini, R. Pittau and A.D. Polosa, ALPGEN, a generator for hard multiparton processes in hadronic collisions, JHEP 07 (2003) 001 [hep-ph/0206293] [INSPIRE].

[41] B.P. Kersevan and E. Richter-Was, The Monte Carlo event generator AcerMC version 2.0 with interfaces to PYTHIA 6.2 and HERWIG 6.5, hep-ph/0405247 [INSPIRE].

[42] ATLAS collaboration, G. Aad et al., Measurement of $t \bar{t}$ production with a veto on additional central jet activity in pp collisions at $\sqrt{s}=7 \mathrm{TeV}$ using the ATLAS detector, Eur. Phys. J. C 72 (2012) 2043 [arXiv:1203.5015] [INSPIRE]. 
[43] ATLAS collaboration, ATLAS tunes of PYTHIA 6 and PYTHIA 8 for MC11, PHYS-PUB-2011-009 (2011).

[44] J. Pumplin et al., New generation of parton distributions with uncertainties from global QCD analysis, JHEP 07 (2002) 012 [hep-ph/0201195] [INSPIRE].

[45] J. Alwall, M. Herquet, F. Maltoni, O. Mattelaer and T. Stelzer, MadGraph 5: Going Beyond, JHEP 06 (2011) 128 [arXiv: 1106.0522] [INSPIRE].

[46] T. Gleisberg et al., Event generation with SHERPA 1.1, JHEP 02 (2009) 007 [arXiv:0811.4622] [INSPIRE].

[47] R. Hamberg, W. van Neerven and T. Matsuura, A Complete calculation of the order $\alpha_{s}^{2}$ correction to the Drell-Yan K factor, Nucl. Phys. B 359 (1991) 343 [Erratum ibid. B 644 (2002) 403] [INSPIRE].

[48] R. Gavin, Y. Li, F. Petriello and S. Quackenbush, W Physics at the LHC with FEWZ 2.1, arXiv: 1201.5896 [INSPIRE].

[49] M. Aliev et al., HATHOR: HAdronic Top and Heavy quarks crOss section calculatoR, Comput. Phys. Commun. 182 (2011) 1034 [arXiv:1007.1327] [InSPIRE].

[50] N. Kidonakis, Two-loop soft anomalous dimensions for single top quark associated production with $a W^{-}$or $H^{-}$, Phys. Rev. D 82 (2010) 054018 [arXiv: 1005.4451] [INSPIRE].

[51] T. Binoth, M. Ciccolini, N. Kauer and M. Krämer, Gluon-induced W-boson pair production at the LHC, JHEP 12 (2006) 046 [hep-ph/0611170] [INSPIRE].

[52] A. Lazopoulos, T. McElmurry, K. Melnikov and F. Petriello, Next-to-leading order QCD corrections to $t \bar{t} Z$ production at the LHC, Phys. Lett. B 666 (2008) 62 [arXiv:0804.2220] [INSPIRE].

[53] M. Bahr et al., HERWIG++ Physics and Manual, Eur. Phys. J. C 58 (2008) 639 [arXiv:0803.0883] [INSPIRE].

[54] W. Beenakker, M. Krämer, T. Plehn, M. Spira and P. Zerwas, Stop production at hadron colliders, Nucl. Phys. B 515 (1998) 3 [hep-ph/9710451] [InSPIRE].

[55] W. Beenakker et al., Supersymmetric top and bottom squark production at hadron colliders, JHEP 08 (2010) 098 [arXiv: 1006.4771] [INSPIRE].

[56] W. Beenakker et al., Squark and Gluino Hadroproduction, Int. J. Mod. Phys. A 26 (2011) 2637 [arXiv: 1105.1110] [INSPIRE].

[57] M. Krämer et al., Supersymmetry production cross sections in pp collisions at $\sqrt{s}=7 \mathrm{TeV}$, arXiv:1206.2892 [INSPIRE].

[58] ATLAS collaboration, First tuning of HERWIG/JIMMY to ATLAS data, PHYS-PUB-2010-014 (2010).

[59] ATLAS Collaboration, Charged particle multiplicities in $p p$ interactions at $\sqrt{s}=0.9$ and $7 \mathrm{TeV}$ in a diffractive limited phase-space measured with the ATLAS detector at the LHC and new PYTHIA6 tune, ATL-CONF-2010-031.

[60] ATLAS collaboration, G. Aad et al., The ATLAS Simulation Infrastructure, Eur. Phys. J. C 70 (2010) 823 [arXiv: 1005.4568] [INSPIRE].

[61] GEANT4 collaboration, S. Agostinelli et al., GEANT4: A Simulation toolkit, Nucl. Instrum. Meth. A 506 (2003) 250 [INSPIRE].

[62] M. Cacciari and G.P. Salam, Dispelling the $N^{3}$ myth for the $k_{t}$ jet-finder, Phys. Lett. B 641 (2006) 57 [hep-ph/0512210] [INSPIRE]. 
[63] M. Cacciari, G.P. Salam and G. Soyez, The Anti-k $k_{t}$ jet clustering algorithm, JHEP 04 (2008) 063 [arXiv: 0802.1189] [INSPIRE].

[64] ATLAS collaboration, G. Aad et al., Jet energy measurement with the ATLAS detector in proton-proton collisions at $\sqrt{s}=7 \mathrm{TeV}$, arXiv:1112.6426 [INSPIRE].

[65] ATLAS collaboration, G. Aad et al., Electron performance measurements with the ATLAS detector using the 2010 LHC proton-proton collision data, Eur. Phys. J. C 72 (2012) 1909 [arXiv:1110.3174] [INSPIRE].

[66] ATLAS collaboration, Muon reconstruction efficiency in reprocessed 2010 LHC proton-proton collision data recorded with the ATLAS detector, ATLAS-CONF-2011-063 (2011).

[67] ATLAS collaboration, G. Aad et al., Performance of Missing Transverse Momentum Reconstruction in Proton-Proton Collisions at $7 \mathrm{TeV}$ with ATLAS, Eur. Phys. J. C 72 (2012) 1844 [arXiv: 1108.5602] [inSPIRE].

[68] ATLAS Collaboration, Commissioning of the ATLAS high-performance b-tagging algorithms in $7 \mathrm{TeV}$ collision data, ATL-CONF-2011-102.

[69] ATLAS Collaboration, Luminosity determination in pp collisions at $\sqrt{s}=7 \mathrm{TeV}$ using the ATLAS detector in 2011, ATL-CONF-2011-116.

[70] ATLAS collaboration, G. Aad et al., Luminosity Determination in pp Collisions at $\sqrt{s}=7$ TeV Using the ATLAS Detector at the LHC, Eur. Phys. J. C 71 (2011) 1630 [arXiv: 1101.2185] [INSPIRE].

[71] ATLAS collaboration, G. Aad et al., Measurement of the top quark-pair production cross section with ATLAS in pp collisions at $\sqrt{s}=7$ TeV, Eur. Phys. J. C 71 (2011) 1577 [arXiv: 1012.1792] [INSPIRE].

[72] ATLAS collaboration, G. Aad et al., Measurement of the top quark pair production cross section in pp collisions at $\sqrt{s}=7 \mathrm{TeV}$ in dilepton final states with ATLAS, Phys. Lett. B 707 (2012) 459 [arXiv:1108.3699] [INSPIRE].

[73] ATLAS Collaboration, Jet energy resolution and reconstruction efficiencies from in-situ techniques with the ATLAS detector using proton-proton collisionsat a center-of-mass energy $\sqrt{s}=7 \mathrm{TeV}$, ATL-CONF-2010-054.

[74] P.M. Nadolsky et al., Implications of CTEQ global analysis for collider observables, Phys. Rev. D 78 (2008) 013004 [arXiv:0802.0007] [INSPIRE].

[75] A. Martin, W. Stirling, R. Thorne and G. Watt, Parton distributions for the LHC, Eur. Phys. J. C 63 (2009) 189 [arXiv:0901.0002] [InSPIRE].

[76] M. Botje et al., The PDF4LHC Working Group Interim Recommendations, arXiv: 1101.0538 [INSPIRE].

[77] A.L. Read, Presentation of search results: The $C L_{s}$ technique, J. Phys. G 28 (2002) 2693 [INSPIRE].

[78] ATLAS collaboration, G. Aad et al., Search for a supersymmetric partner to the top quark in final states with jets and missing transverse momentum at $\sqrt{s}=7 \mathrm{TeV}$ with the ATLAS detector, arXiv:1208.1447 [INSPIRE].

[79] ATLAS collaboration, G. Aad et al., Search for direct top squark pair production in final states with one isolated lepton, jets and missing transverse momentum in $\sqrt{s}=7 \mathrm{TeV} p p$ collisions using $4.7 \mathrm{fb}^{-1}$ of ATLAS data, arXiv:1208.2590 [INSPIRE]. 


\section{The ATLAS collaboration}

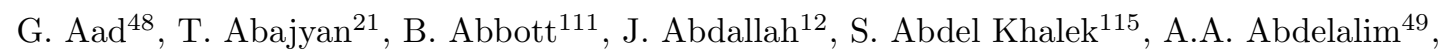
O. Abdinov ${ }^{11}$, R. Aben ${ }^{105}$, B. Abi ${ }^{112}$, M. Abolins ${ }^{88}$, O.S. AbouZeid ${ }^{158}$, H. Abramowicz ${ }^{153}$, H. Abreu ${ }^{136}$, E. Acerbi ${ }^{89 a, 89 b}$, B.S. Acharya ${ }^{164 a, 164 b}$, L. Adamczyk ${ }^{38}$, D.L. Adams ${ }^{25}$, T.N. Addy ${ }^{56}$, J. Adelman ${ }^{176}$, S. Adomeit ${ }^{98}$, P. Adragna ${ }^{75}$, T. Adye ${ }^{129}$, S. Aefsky ${ }^{23}$, J.A. Aguilar-Saavedra ${ }^{124 b, a}$, M. Agustoni ${ }^{17}$, M. Aharrouche ${ }^{81}$, S.P. Ahlen ${ }^{22}$, F. Ahles ${ }^{48}$, A. Ahmad ${ }^{148}$, M. Ahsan ${ }^{41}$, G. Aielli133a,133b, T. Akdogan ${ }^{19 a}$, T.P.A. Åkesson ${ }^{79}$, G. Akimoto ${ }^{155}$, A.V. Akimov ${ }^{94}$, M.S. Alam ${ }^{2}$, M.A. Alam ${ }^{76}$, J. Albert ${ }^{169}$, S. Albrand ${ }^{55}$, M. Aleksa ${ }^{30}$,

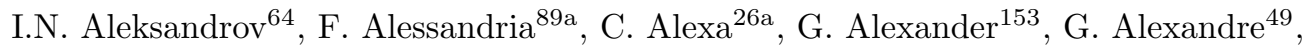
T. Alexopoulos ${ }^{10}$, M. Alhroob ${ }^{164 a, 164 c}$, M. Aliev ${ }^{16}$, G. Alimonti ${ }^{89 a}$, J. Alison ${ }^{120}$,

B.M.M. Allbrooke ${ }^{18}$, P.P. Allport ${ }^{73}$, S.E. Allwood-Spiers ${ }^{53}$, J. Almond ${ }^{82}$, A. Aloisio ${ }^{102 a, 102 b}$, R. Alon ${ }^{172}$, A. Alonso ${ }^{79}$, F. Alonso ${ }^{70}$, B. Alvarez Gonzalez ${ }^{88}$, M.G. Alviggi $^{102 a, 102 b}$, K. Amako ${ }^{65}$, C. Amelung ${ }^{23}$, V.V. Ammosov ${ }^{128, *}$, A. Amorim ${ }^{124 a, b}$, N. Amram ${ }^{153}$, C. Anastopoulos ${ }^{30}$, L.S. Ancu ${ }^{17}$, N. Andari ${ }^{115}$, T. Andeen ${ }^{35}$, C.F. Anders ${ }^{58 b}$, G. Anders ${ }^{58 a}$, K.J. Anderson ${ }^{31}$, A. Andreazza ${ }^{89 a, 89 b}$, V. Andrei ${ }^{58 a}$, X.S. Anduaga ${ }^{70}$, P. Anger ${ }^{44}$, A. Angerami ${ }^{35}$, F. Anghinolfi ${ }^{30}$, A. Anisenkov ${ }^{107}$, N. Anjos ${ }^{124 a}$, A. Annovi ${ }^{47}$, A. Antonaki ${ }^{9}$, M. Antonelli ${ }^{47}$, A. Antonov ${ }^{96}$, J. Antos ${ }^{144 b}$, F. Anulli132a, M. Aoki ${ }^{101}$, S. Aoun ${ }^{83}$, L. Aperio Bella ${ }^{5}$, R. Apolle ${ }^{118, c}$, G. Arabidze ${ }^{88}$, I. Aracena ${ }^{143}$, Y. Arai ${ }^{65}$, A.T.H. Arce ${ }^{45}$, S. Arfaoui ${ }^{148}$, J-F. Arguin ${ }^{15}$, E. Arik ${ }^{19 a, *}$, M. Arik ${ }^{19 a}$, A.J. Armbruster ${ }^{87}$, O. Arnaez ${ }^{81}$, V. Arnal ${ }^{80}$, C. Arnault ${ }^{115}$, A. Artamonov ${ }^{95}$, G. Artoni ${ }^{132 a, 132 b}$, D. Arutinov ${ }^{21}$, S. Asai ${ }^{155}$, R. Asfandiyarov ${ }^{173}$, S. Ask ${ }^{28}$, B. Åsman ${ }^{146 a, 146 b}$, L. Asquith ${ }^{6}$, K. Assamagan ${ }^{25}$, A. Astbury ${ }^{169}$, M. Atkinson ${ }^{165}$, B. Aubert ${ }^{5}$, E. Auge ${ }^{115}$, K. Augsten ${ }^{127}$, M. Aurousseau ${ }^{145 a}$, G. Avolio ${ }^{163}$, R. Avramidou ${ }^{10}$, D. Axen ${ }^{168}$, G. Azuelos ${ }^{93, d}$, Y. Azuma ${ }^{155}$, M.A. Baak ${ }^{30}$, G. Baccaglioni ${ }^{89 a}$, C. Bacci ${ }^{134 a, 134 b}$, A.M. Bach ${ }^{15}$, H. Bachacou ${ }^{136}$, K. Bachas ${ }^{30}$, M. Backes ${ }^{49}$, M. Backhaus ${ }^{21}$, E. Badescu ${ }^{26 a}$, P. Bagnaia 132a,132b, S. Bahinipati ${ }^{3}$, Y. Bai ${ }^{33 a}$, D.C. Bailey ${ }^{158}$, T. Bain ${ }^{158}$, J.T. Baines ${ }^{129}$, O.K. Baker ${ }^{176}$, M.D. Baker ${ }^{25}$, S. Baker ${ }^{77}$, E. Banas ${ }^{39}$, P. Banerjee ${ }^{93}$, Sw. Banerjee ${ }^{173}$, D. Banfi ${ }^{30}$, A. Bangert ${ }^{150}$, V. Bansal ${ }^{169}$, H.S. Bansil ${ }^{18}$, L. Barak ${ }^{172}$, S.P. Baranov ${ }^{94}$, A. Barbaro Galtieri ${ }^{15}$, T. Barber ${ }^{48}$, E.L. Barberio ${ }^{86}$, D. Barberis ${ }^{50 a, 50 b}$, M. Barbero ${ }^{21}$, D.Y. Bardin ${ }^{64}$, T. Barillari ${ }^{99}$, M. Barisonzi ${ }^{175}$, T. Barklow ${ }^{143}$, N. Barlow ${ }^{28}$, B.M. Barnett ${ }^{129}$, R.M. Barnett ${ }^{15}$, A. Baroncelli ${ }^{134 a}$, G. Barone ${ }^{49}$, A.J. Barr ${ }^{118}$, F. Barreiro ${ }^{80}$, J. Barreiro Guimarães da $\operatorname{Costa}^{57}$, P. Barrillon ${ }^{115}$, R. Bartoldus ${ }^{143}$, A.E. Barton ${ }^{71}$, V. Bartsch ${ }^{149}$, A. Basye ${ }^{165}$, R.L. Bates ${ }^{53}$, L. Batkova ${ }^{144 a}$, J.R. Batley ${ }^{28}$, A. Battaglia ${ }^{17}$, M. Battistin ${ }^{30}$, F. Bauer ${ }^{136}$, H.S. Bawa ${ }^{143, e}$, S. Beale ${ }^{98}$, T. Beau ${ }^{78}$, P.H. Beauchemin ${ }^{161}$, R. Beccherle ${ }^{50 a}$, P. Bechtle ${ }^{21}$, H.P. Beck ${ }^{17}$, A.K. Becker ${ }^{175}$, S. Becker ${ }^{98}$, M. Beckingham ${ }^{138}$, K.H. Becks ${ }^{175}$, A.J. Beddall ${ }^{19 c}$, A. Beddall ${ }^{19 c}$, S. Bedikian ${ }^{176}$, V.A. Bednyakov ${ }^{64}$, C.P. Bee ${ }^{83}$, L.J. Beemster ${ }^{105}$, M. Begel ${ }^{25}$, S. Behar Harpaz ${ }^{152}$, M. Beimforde ${ }^{99}$, C. Belanger-Champagne ${ }^{85}$, P.J. Bell ${ }^{49}$, W.H. Bell ${ }^{49}$, G. Bella ${ }^{153}$, L. Bellagamba ${ }^{20 a}$, F. Bellina ${ }^{30}$, M. Bellomo $^{30}$, A. Belloni ${ }^{57}$, O. Beloborodova ${ }^{107, f}$, K. Belotskiy ${ }^{96}$, O. Beltramello ${ }^{30}$, O. Benary ${ }^{153}$, D. Benchekroun ${ }^{135 a}$, K. Bendtz ${ }^{146 a, 146 b}$, N. Benekos ${ }^{165}$, Y. Benhammou ${ }^{153}$, E. Benhar Noccioli ${ }^{49}$, J.A. Benitez Garcia ${ }^{159 b}$, D.P. Benjamin ${ }^{45}$, M. Benoit ${ }^{115}$, J.R. Bensinger ${ }^{23}$, K. Benslama ${ }^{130}$, S. Bentvelsen ${ }^{105}$, D. Berge ${ }^{30}$, E. Bergeaas Kuutmann ${ }^{42}$, N. Berger ${ }^{5}$, F. Berghaus ${ }^{169}$, E. Berglund ${ }^{105}$, J. Beringer ${ }^{15}$, P. Bernat ${ }^{77}$, R. Bernhard ${ }^{48}$, C. Bernius ${ }^{25}$, T. Berry ${ }^{76}$, C. Bertella ${ }^{83}$, A. Bertin ${ }^{20 a, 20 b}$, F. Bertolucci ${ }^{122 a, 122 b}$, M.I. Besana ${ }^{89 a, 89 b}$, G.J. Besjes ${ }^{104}$, N. Besson ${ }^{136}$, S. Bethke ${ }^{99}$, W. Bhimji ${ }^{46}$, R.M. Bianchi ${ }^{30}$, M. Bianco ${ }^{72 a}, 72 b$, O. Biebel ${ }^{98}$, S.P. Bieniek ${ }^{77}$, K. Bierwagen ${ }^{54}$, J. Biesiada ${ }^{15}$, M. Biglietti134a ${ }^{13}$, H. Bilokon ${ }^{47}$, M. Bindi ${ }^{20 a, 20 b}$, S. Binet ${ }^{115}$, A. Bingul ${ }^{19 c}$, C. Bini ${ }^{132 a, 132 b}$, C. Biscarat ${ }^{178}$, B. Bittner ${ }^{99}$, K.M. Black ${ }^{22}$, R.E. Blair ${ }^{6}$, J.-B. Blanchard ${ }^{136}$, G. Blanchot ${ }^{30}$, T. Blazek ${ }^{144 a}$, C. Blocker ${ }^{23}$, J. Blocki ${ }^{39}$, A. Blondel ${ }^{49}$, W. Blum ${ }^{81}$, U. Blumenschein ${ }^{54}$, G.J. Bobbink ${ }^{105}$, V.B. Bobrovnikov ${ }^{107}$, S.S. Bocchetta ${ }^{79}$, 
A. Bocci ${ }^{45}$, C.R. Boddy ${ }^{118}$, M. Boehler ${ }^{48}$, J. Boek ${ }^{175}$, N. Boelaert ${ }^{36}$, J.A. Bogaerts ${ }^{30}$, A. Bogdanchikov ${ }^{107}$, A. Bogouch ${ }^{90, *}$, C. Bohm ${ }^{146 a}$, J. Bohm ${ }^{125}$, V. Boisvert ${ }^{76}$, T. Bold ${ }^{38}$, V. Boldea ${ }^{26 a}$, N.M. Bolnet ${ }^{136}$, M. Bomben ${ }^{78}$, M. Bona ${ }^{75}$, M. Boonekamp ${ }^{136}$, C.N. Booth ${ }^{139}$, S. Bordoni ${ }^{78}$, C. Borer ${ }^{17}$, A. Borisov ${ }^{128}$, G. Borissov ${ }^{71}$, I. Borjanovic ${ }^{13 a}$, M. Borri ${ }^{82}$, S. Borroni ${ }^{87}$,

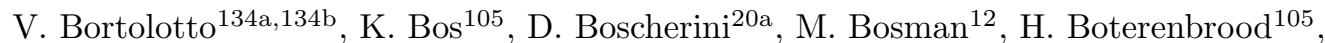
J. Bouchami ${ }^{93}$, J. Boudreau ${ }^{123}$, E.V. Bouhova-Thacker ${ }^{71}$, D. Boumediene ${ }^{34}$, C. Bourdarios ${ }^{115}$, N. Bousson ${ }^{83}$, A. Boveia ${ }^{31}$, J. Boyd ${ }^{30}$, I.R. Boyko ${ }^{64}$, I. Bozovic-Jelisavcic ${ }^{13 b}$, J. Bracinik ${ }^{18}$, P. Branchini ${ }^{134 a}$, A. Brandt ${ }^{8}$, G. Brandt ${ }^{118}$, O. Brandt ${ }^{54}$, U. Bratzler ${ }^{156}$, B. Brau ${ }^{84}$, J.E. Brau ${ }^{114}$, H.M. Braun ${ }^{175, *}$, S.F. Brazzale ${ }^{164 a, 164 c}$, B. Brelier ${ }^{158}$, J. Bremer ${ }^{30}$, K. Brendlinger ${ }^{120}$, R. Brenner ${ }^{166}$, S. Bressler ${ }^{172}$, D. Britton ${ }^{53}$, F.M. Brochu ${ }^{28}$, I. Brock ${ }^{21}$, R. Brock ${ }^{88}$, F. Broggi ${ }^{89 a}$, C. Bromberg ${ }^{88}$, J. Bronner ${ }^{99}$, G. Brooijmans ${ }^{35}$, T. Brooks ${ }^{76}$, W.K. Brooks ${ }^{32 b}$, G. Brown ${ }^{82}$, H. Brown ${ }^{8}$, P.A. Bruckman de Renstrom ${ }^{39}$, D. Bruncko ${ }^{144 b}$, R. Bruneliere ${ }^{48}$, S. Brunet ${ }^{60}$,

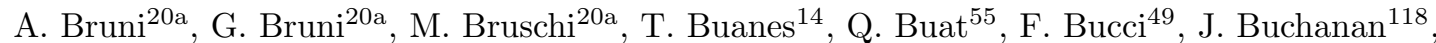
P. Buchholz ${ }^{141}$, R.M. Buckingham ${ }^{118}$, A.G. Buckley ${ }^{46}$, S.I. Buda ${ }^{26 a}$, I.A. Budagov ${ }^{64}$, B. Budick ${ }^{108}$, V. Büscher ${ }^{81}$, L. Bugge ${ }^{117}$, O. Bulekov ${ }^{96}$, A.C. Bundock ${ }^{73}$, M. Bunse ${ }^{43}$, T. Buran ${ }^{117}$, H. Burckhart ${ }^{30}$, S. Burdin ${ }^{73}$, T. Burgess ${ }^{14}$, S. Burke ${ }^{129}$, E. Busato ${ }^{34}$, P. Bussey ${ }^{53}$, C.P. Buszello ${ }^{166}$, B. Butler ${ }^{143}$, J.M. Butler ${ }^{22}$, C.M. Buttar ${ }^{53}$, J.M. Butterworth ${ }^{77}$, W. Buttinger ${ }^{28}$, S. Cabrera Urbán ${ }^{167}$, D. Caforio ${ }^{20 a}, 20 b$, O. Cakir ${ }^{4 a}$, P. Calafiura ${ }^{15}$, G. Calderini ${ }^{78}$, P. Calfayan ${ }^{98}$, R. Calkins ${ }^{106}$, L.P. Caloba ${ }^{24 a}$, R. Caloi ${ }^{132 a, 132 b}$, D. Calvet ${ }^{34}$, S. Calvet ${ }^{34}$, R. Camacho Toro $^{34}$, P. Camarri ${ }^{133 a, 133 b}$, D. Cameron ${ }^{117}$, L.M. Caminada ${ }^{15}$, R. Caminal Armadans ${ }^{12}$, S. Campana ${ }^{30}$, M. Campanelli ${ }^{77}$, V. Canale ${ }^{102 a, 102 b}$, F. Canelli ${ }^{31, g}$, A. Canepa ${ }^{159 a}$, J. Cantero ${ }^{80}$, R. Cantrill ${ }^{76}$, L. Capasso ${ }^{102 a, 102 b}$, M.D.M. Capeans Garrido ${ }^{30}$, I. Caprini26a ${ }^{26}$, M. Caprini ${ }^{26 a}$, D. Capriotti ${ }^{99}$, M. Capua ${ }^{37 a, 37 b}$, R. Caputo $^{81}$, R. Cardarelli ${ }^{133 a}$, T. Carli $^{30}$, G. Carlino ${ }^{102 a}$, L. Carminati ${ }^{89 a, 89 b}$, B. Caron $^{85}$, S. Caron ${ }^{104}$, E. Carquin ${ }^{32 b}$, G.D. Carrillo Montoya ${ }^{173}$, A.A. Carter ${ }^{75}$, J.R. Carter ${ }^{28}$, J. Carvalho ${ }^{124 a, h}$, D. Casadei ${ }^{108}$, M.P. Casado ${ }^{12}$, M. Cascella ${ }^{122 a, 122 b}$, C. Caso ${ }^{50 a, 50 b, *}$, A.M. Castaneda Hernandez ${ }^{173, i}$, E. Castaneda-Miranda ${ }^{173}$, V. Castillo Gimenez ${ }^{167}$, N.F. Castro ${ }^{124 a}$, G. Cataldi ${ }^{72 a}$, P. Catastini ${ }^{57}$, A. Catinaccio ${ }^{30}$, J.R. Catmore ${ }^{30}$, A. Cattai ${ }^{30}$,

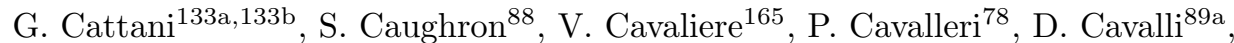
M. Cavalli-Sforza ${ }^{12}$, V. Cavasinni ${ }^{122 a, 122 b}$, F. Ceradini ${ }^{134 a, 134 b}$, A.S. Cerqueira ${ }^{24 b}$, A. Cerri ${ }^{30}$, L. Cerrito ${ }^{75}$, F. Cerutti ${ }^{47}$, S.A. Cetin ${ }^{19 b}$, A. Chafaq ${ }^{135 a}$, D. Chakraborty ${ }^{106}$, I. Chalupkova ${ }^{126}$, K. Chan ${ }^{3}$, P. Chang ${ }^{165}$, B. Chapleau ${ }^{85}$, J.D. Chapman ${ }^{28}$, J.W. Chapman ${ }^{87}$, E. Chareyre ${ }^{78}$, D.G. Charlton ${ }^{18}$, V. Chavda ${ }^{82}$, C.A. Chavez Barajas ${ }^{30}$, S. Cheatham ${ }^{85}$, S. Chekanov ${ }^{6}$, S.V. Chekulaev ${ }^{159 a}$, G.A. Chelkov ${ }^{64}$, M.A. Chelstowska ${ }^{104}$, C. Chen ${ }^{63}$, H. Chen ${ }^{25}$, S. Chen ${ }^{33 c}$, X. Chen ${ }^{173}$, Y. Chen ${ }^{35}$, A. Cheplakov ${ }^{64}$, R. Cherkaoui El Moursli ${ }^{135 e}$, V. Chernyatin ${ }^{25}$, E. Cheu ${ }^{7}$, S.L. Cheung ${ }^{158}$, L. Chevalier ${ }^{136}$, G. Chiefari ${ }^{102 a, 102 b}$, L. Chikovani ${ }^{51 a, *}$, J.T. Childers ${ }^{30}$, A. Chilingarov ${ }^{71}$, G. Chiodini ${ }^{72 a}$, A.S. Chisholm ${ }^{18}$, R.T. Chislett ${ }^{77}$, A. Chitan ${ }^{26 a}$, M.V. Chizhov ${ }^{64}$, G. Choudalakis ${ }^{31}$, S. Chouridou ${ }^{137}$, I.A. Christidi ${ }^{77}$, A. Christov ${ }^{48}$, D. Chromek-Burckhart ${ }^{30}$, M.L. Chu ${ }^{151}$, J. Chudoba ${ }^{125}$, G. Ciapetti ${ }^{132 a, 132 b}$, A.K. Ciftci $^{4 a}$,

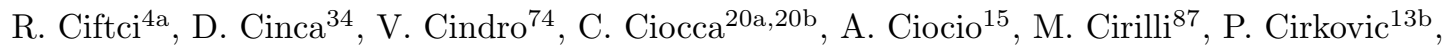
M. Citterio ${ }^{89 a}$, M. Ciubancan ${ }^{26 a}$, A. Clark ${ }^{49}$, P.J. Clark $^{46}$, R.N. Clarke ${ }^{15}$, W. Cleland ${ }^{123}$, J.C. Clemens ${ }^{83}$, B. Clement ${ }^{55}$, C. Clement ${ }^{146 a, 146 b}$, Y. Coadou ${ }^{83}$, M. Cobal ${ }^{164 a, 164 c}$, A. Coccaro ${ }^{138}$, J. Cochran ${ }^{63}$, J.G. Cogan ${ }^{143}$, J. Coggeshall ${ }^{165}$, E. Cogneras ${ }^{178}$, J. Colas ${ }^{5}$, S. Cole ${ }^{106}$, A.P. Colijn ${ }^{105}$, N.J. Collins ${ }^{18}$, C. Collins-Tooth ${ }^{53}$, J. Collot ${ }^{55}$, T. Colombo ${ }^{119 a, 119 b}$, G. Colon $^{84}$, P. Conde Muiño ${ }^{124 a}$, E. Coniavitis ${ }^{118}$, M.C. Conidii ${ }^{12}$, S.M. Consonni ${ }^{89 a}, 89 b$, V. Consorti ${ }^{48}$, S. Constantinescu ${ }^{26 a}$, C. Conta ${ }^{119 a, 119 b}$, G. Conti ${ }^{57}$, F. Conventi ${ }^{102 a, j}$, M. Cooke $^{15}$, B.D. Cooper ${ }^{77}$, A.M. Cooper-Sarkar ${ }^{118}$, K. Copic ${ }^{15}$, T. Cornelissen ${ }^{175}$, M. Corradi ${ }^{20 a}$, F. Corriveau ${ }^{85, k}$, A. Cortes-Gonzalez ${ }^{165}$, G. Cortiana ${ }^{99}$, G. Costa ${ }^{89 a}$, M.J. Costa ${ }^{167}$,

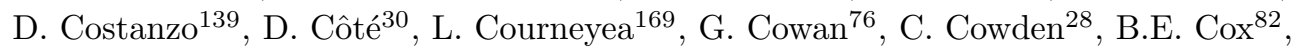


K. Cranmer ${ }^{108}$, F. Crescioli ${ }^{122 a, 122 b}$, M. Cristinziani ${ }^{21}$, G. Crosetti ${ }^{37 a, 37 b}$, S. Crépé-Renaudin ${ }^{55}$, C.-M. Cuciucc ${ }^{26 a}$, C. Cuenca Almenar ${ }^{176}$, T. Cuhadar Donszelmann ${ }^{139}$, M. Curatolo ${ }^{47}$, C.J. Curtis ${ }^{18}$, C. Cuthbert ${ }^{150}$, P. Cwetanski ${ }^{60}$, H. Czirr ${ }^{141}$, P. Czodrowski ${ }^{44}$, Z. Czyczula ${ }^{176}$, S. D'Auria ${ }^{53}$, M. D'Onofrio ${ }^{73}$, A. D'Orazio ${ }^{132 a, 132 b}$, M.J. Da Cunha Sargedas De Sousa ${ }^{124 a}$, C. Da Via ${ }^{82}$, W. Dabrowski ${ }^{38}$, A. Dafinca ${ }^{118}$, T. Dai ${ }^{87}$, C. Dallapiccola ${ }^{84}$, M. Dam ${ }^{36}$, M. Dameri ${ }^{50 a, 50 b}$, D.S. Damiani ${ }^{137}$, H.O. Danielsson ${ }^{30}$, V. Dao ${ }^{49}$, G. Darbo ${ }^{50 a}$, G.L. Darlea ${ }^{26 b}$, J.A. Dassoulas ${ }^{42}$, W. Davey ${ }^{21}$, T. Davidek ${ }^{126}$, N. Davidson ${ }^{86}$, R. Davidson ${ }^{71}$, E. Davies ${ }^{118, c}$, M. Davies ${ }^{93}$, O. Davignon ${ }^{78}$, A.R. Davison ${ }^{77}$, Y. Davygora ${ }^{58 a}$, E. Dawe ${ }^{142}$, I. Dawson ${ }^{139}$, R.K. Daya-Ishmukhametova ${ }^{23}$, K. De ${ }^{8}$, R. de Asmundis ${ }^{102 a}$, S. De Castro ${ }^{20 a, 20 b}$, S. De Cecco ${ }^{78}$, J. de Graat ${ }^{98}$, N. De Groot ${ }^{104}$, P. de Jong ${ }^{105}$, C. De La Taille ${ }^{115}$, H. De la Torre ${ }^{80}$, F. De Lorenzi ${ }^{63}$, L. de Mora ${ }^{71}$, L. De Nooij ${ }^{105}$, D. De Pedis ${ }^{132 a}$, A. De Salvo ${ }^{132 a}$, U. De Sanctis ${ }^{164 a, 164 c}$, A. De Santo ${ }^{149}$, J.B. De Vivie De Regie ${ }^{115}$, G. De Zorzi ${ }^{132 a, 132 b}$, W.J. Dearnaley ${ }^{71}$, R. Debbe ${ }^{25}$, C. Debenedetti ${ }^{46}$, B. Dechenaux ${ }^{55}$, D.V. Dedovich ${ }^{64}$, J. Degenhardt ${ }^{120}$, C. Del Papa ${ }^{164 a, 164 c}$, J. Del Peso ${ }^{80}$, T. Del Prete ${ }^{122 a, 122 b}$, T. Delemontex ${ }^{55}$, M. Deliyergiyev ${ }^{74}$, A. Dell'Acqua ${ }^{30}$, L. Dell'Asta ${ }^{22}$, M. Della Pietra ${ }^{102 a, j}$, D. della Volpe ${ }^{102 a, 102 b}$, M. Delmastro ${ }^{5}$, P.A. Delsart ${ }^{55}$, C. Deluca ${ }^{105}$, S. Demers ${ }^{176}$, M. Demichev ${ }^{64}$, B. Demirkoz ${ }^{12, l}$, J. Deng ${ }^{163}$, S.P. Denisov ${ }^{128}$, D. Derendarz ${ }^{39}$, J.E. Derkaoui ${ }^{135 d}$, F. Derue ${ }^{78}$, P. Dervan ${ }^{73}$, K. Desch ${ }^{21}$, E. Devetak ${ }^{148}$, P.O. Deviveiros ${ }^{105}$, A. Dewhurst ${ }^{129}$, B. DeWilde ${ }^{148}$, S. Dhaliwal ${ }^{158}$, R. Dhullipudi ${ }^{25, m}$, A. Di Ciaccio ${ }^{133 a, 133 b}$, L. Di $\mathrm{Ciaccio}^{5}$, A. Di Girolamo ${ }^{30}$, B. Di Girolamo ${ }^{30}$, S. Di Luise ${ }^{134 a, 134 b}$, A. Di Mattia ${ }^{173}$, B. Di Micco ${ }^{30}$, R. Di Nardo ${ }^{47}$, A. Di Simone ${ }^{133 a, 133 b}$, R. Di Sipio ${ }^{20 a, 20 b}$, M.A. Diaz ${ }^{32 a}$, E.B. Diehl ${ }^{87}$, J. Dietrich ${ }^{42}$, T.A. Dietzsch ${ }^{58 a}$, S. Diglio ${ }^{86}$,

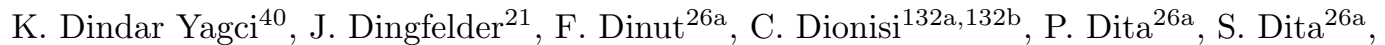
F. Dittus ${ }^{30}$, F. Djama ${ }^{83}$, T. Djobava ${ }^{51 b}$, M.A.B. do Vale ${ }^{24 c}$, A. Do Valle Wemans ${ }^{124 a, n}$,

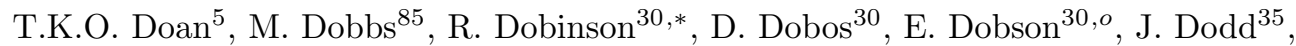
C. Doglioni ${ }^{49}$, T. Doherty ${ }^{53}$, Y. Doi ${ }^{65, *}$, J. Dolejsi ${ }^{126}$, I. Dolenc ${ }^{74}$, Z. Dolezal ${ }^{126}$, B.A. Dolgoshein ${ }^{96, *}$, T. Dohmae ${ }^{155}$, M. Donadelli24d, J. Donini ${ }^{34}$, J. Dopke ${ }^{30}$, A. Doria ${ }^{102 a}$, A. Dos Anjos ${ }^{173}$, A. Dotti ${ }^{122 a, 122 b}$, M.T. Dova ${ }^{70}$, A.D. Doxiadis ${ }^{105}$, A.T. Doyle ${ }^{53}$, M. Dris ${ }^{10}$, J. Dubbert ${ }^{99}$, S. Dube ${ }^{15}$, E. Duchovni ${ }^{172}$, G. Duckeck ${ }^{98}$, D. Duda ${ }^{175}$, A. Dudarev ${ }^{30}$, F. Dudziak ${ }^{63}$,

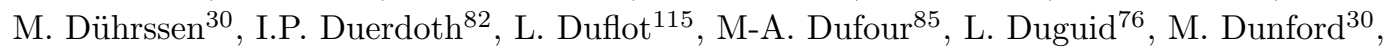
H. Duran Yildiz ${ }^{4 a}$, R. Duxfield ${ }^{139}$, M. Dwuznik ${ }^{38}$, F. Dydak ${ }^{30}$, M. Düren ${ }^{52}$, J. Ebke ${ }^{98}$, S. Eckweiler ${ }^{81}$, K. Edmonds ${ }^{81}$, W. Edson ${ }^{2}$, C.A. Edwards ${ }^{76}$, N.C. Edwards ${ }^{53}$, W. Ehrenfeld ${ }^{42}$, T. Eifert ${ }^{143}$, G. Eigen ${ }^{14}$, K. Einsweiler ${ }^{15}$, E. Eisenhandler ${ }^{75}$, T. Ekelof ${ }^{166}$, M. El Kacimi ${ }^{135 c}$, M. Ellert ${ }^{166}$, S. Elles $^{5}$, F. Ellinghaus ${ }^{81}$, K. Ellis ${ }^{75}$, N. Ellis ${ }^{30}$, J. Elmsheuser ${ }^{98}$, M. Elsing ${ }^{30}$, D. Emeliyanov ${ }^{129}$, R. Engelmann ${ }^{148}$, A. Engl ${ }^{98}$, B. Epp ${ }^{61}$, J. Erdmann ${ }^{54}$, A. Ereditato ${ }^{17}$, D. Eriksson ${ }^{146 a}$, J. Ernst ${ }^{2}$, M. Ernst ${ }^{25}$, J. Ernwein ${ }^{136}$, D. Errede ${ }^{165}$, S. Errede ${ }^{165}$, E. Ertel ${ }^{81}$, M. Escalier ${ }^{115}$, H. Esch $^{43}$, C. Escobar ${ }^{123}$, X. Espinal Curull ${ }^{12}$, B. Esposito ${ }^{47}$, F. Etienne ${ }^{83}$, A.I. Etienvre ${ }^{136}$, E. Etzion ${ }^{153}$, D. Evangelakou ${ }^{54}$, H. Evans $^{60}$, L. Fabbri ${ }^{20 a, 20 b}$, C. Fabre $^{30}$, R.M. Fakhrutdinov ${ }^{128}$, S. Falciano ${ }^{132 a}$, Y. Fang ${ }^{173}$, M. Fanti ${ }^{89 a, 89 b}$, A. Farbin ${ }^{8}$, A. Farilla ${ }^{134 a}$, J. Farley ${ }^{148}$, T. Farooque ${ }^{158}$, S. Farrell ${ }^{163}$, S.M. Farrington ${ }^{170}$, P. Farthouat ${ }^{30}$, F. Fassi ${ }^{167}$, P. Fassnacht ${ }^{30}$, D. Fassouliotis ${ }^{9}$, B. Fatholahzadeh ${ }^{158}$, A. Favareto ${ }^{89 a, 89 b}$, L. Fayard ${ }^{115}$, S. Fazio ${ }^{37 a, 37 b}$, R. Febbraro ${ }^{34}$, P. Federic ${ }^{144 a}$, O.L. Fedin ${ }^{121}$, W. Fedorko ${ }^{88}$, M. Fehling-Kaschek ${ }^{48}$, L. Feligioni ${ }^{83}$, D. Fellmann ${ }^{6}$, C. Feng ${ }^{33 d}$, E.J. Feng ${ }^{6}$, A.B. Fenyuk ${ }^{128}$, J. Ferencei ${ }^{144 b}$, W. Fernando ${ }^{6}$, S. Ferrag ${ }^{53}$, J. Ferrando ${ }^{53}$, V. Ferrara ${ }^{42}$, A. Ferrari ${ }^{166}$, P. Ferrari ${ }^{105}$, R. Ferrari ${ }^{119 a}$, D.E. Ferreira de Lima $^{53}$, A. Ferrer ${ }^{167}$, D. Ferrere ${ }^{49}$, C. Ferretti ${ }^{87}$, A. Ferretto Parodi ${ }^{50 a, 50 b}$, M. Fiascaris ${ }^{31}$, F. Fiedler ${ }^{81}$, A. Filipčic ${ }^{74}$, F. Filthaut ${ }^{104}$, M. Fincke-Keeler ${ }^{169}$, M.C.N. Fiolhais ${ }^{124 a, h}$, L. Fiorini ${ }^{167}$, A. Firan ${ }^{40}$, G. Fischer ${ }^{42}$, M.J. Fisher ${ }^{109}$, M. Flechl ${ }^{48}$, I. Fleck ${ }^{141}$, J. Fleckner ${ }^{81}$, P. Fleischmann ${ }^{174}$, S. Fleischmann ${ }^{175}$, T. Flick ${ }^{175}$, A. Floderus ${ }^{79}$, L.R. Flores Castillo ${ }^{173}$, M.J. Flowerdew ${ }^{99}$, T. Fonseca Martin ${ }^{17}$, A. Formica ${ }^{136}$, A. Forti ${ }^{82}$, 
D. Fortin ${ }^{159 a}$, D. Fournier ${ }^{115}$, H. Fox ${ }^{71}$, P. Francavilla ${ }^{12}$, M. Franchini ${ }^{20 a, 20 b}$, S. Franchino 119a,119b , D. Francis ${ }^{30}$, T. Frank ${ }^{172}$, S. Franz ${ }^{30}$, M. Fraternali ${ }^{119 a, 119 b}$, S. Fratina ${ }^{120}$, S.T. French ${ }^{28}$, C. Friedrich ${ }^{42}$, F. Friedrich ${ }^{44}$, R. Froeschl ${ }^{30}$, D. Froidevaux ${ }^{30}$, J.A. Frost ${ }^{28}$, C. Fukunaga ${ }^{156}$, E. Fullana Torregrosa ${ }^{30}$, B.G. Fulsom ${ }^{143}$, J. Fuster ${ }^{167}$, C. Gabaldon ${ }^{30}$, O. Gabizon ${ }^{172}$, T. Gadfort ${ }^{25}$, S. Gadomski ${ }^{49}$, G. Gagliardi ${ }^{50 a, 50 b}$, P. Gagnon ${ }^{60}$, C. Galea ${ }^{98}$, E.J. Gallas ${ }^{118}$, V. Gallo ${ }^{17}$, B.J. Gallop ${ }^{129}$, P. Gallus ${ }^{125}$, K.K. Gan ${ }^{109}$, Y.S. Gao ${ }^{143, e}$, A. Gaponenko ${ }^{15}$, F. Garberson ${ }^{176}$, M. Garcia-Sciveres ${ }^{15}$, C. García ${ }^{167}$, J.E. García Navarro ${ }^{167}$, R.W. Gardner ${ }^{31}$, N. Garelli ${ }^{30}$, H. Garitaonandia ${ }^{105}$, V. Garonne ${ }^{30}$, C. Gatti ${ }^{47}$, G. Gaudio ${ }^{119 a}$, B. Gaur ${ }^{141}$, L. Gauthier ${ }^{136}$, P. Gauzzi ${ }^{132 a, 132 b}$, I.L. Gavrilenko ${ }^{94}$, C. Gay ${ }^{168}$, G. Gaycken ${ }^{21}$, E.N. Gazis ${ }^{10}$, P. Ge ${ }^{33 d}$, Z. Gecse ${ }^{168}$, C.N.P. Gee ${ }^{129}$, D.A.A. Geerts ${ }^{105}$, Ch. Geich-Gimbel ${ }^{21}$, K. Gellerstedt ${ }^{146 a, 146 b}$, C. Gemme ${ }^{50 a}$, A. Gemmell ${ }^{53}$, M.H. Genest ${ }^{55}$, S. Gentile ${ }^{132 a, 132 b}$, M. George ${ }^{54}$, S. George ${ }^{76}$, P. Gerlach ${ }^{175}$, A. Gershon ${ }^{153}$, C. Geweniger ${ }^{58 a}$, H. Ghazlane ${ }^{135 b}$, N. Ghodbane ${ }^{34}$, B. Giacobbe ${ }^{20 a}$, S. Giagu ${ }^{132 a, 132 b}$, V. Giakoumopoulou ${ }^{9}$, V. Giangiobbe ${ }^{12}$, F. Gianotti ${ }^{30}$, B. Gibbard ${ }^{25}$, A. Gibson ${ }^{158}$, S.M. Gibson ${ }^{30}$, D. Gillberg ${ }^{29}$, A.R. Gillman ${ }^{129}$, D.M. Gingrich ${ }^{3, d}$, J. Ginzburg ${ }^{153}$, N. Giokaris ${ }^{9}$, M.P. Giordani ${ }^{164 c}$, R. Giordano ${ }^{102 a, 102 b}$, F.M. Giorgi ${ }^{16}$, P. Giovannini ${ }^{99}$, P.F. Giraud ${ }^{136}$, D. Giugni ${ }^{89 a}$, M. Giunta ${ }^{93}$, P. Giusti ${ }^{20 a}$,

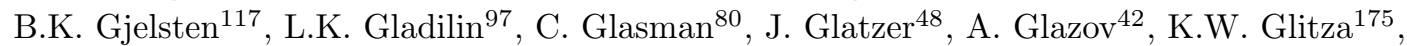
G.L. Glonti ${ }^{64}$, J.R. Goddard ${ }^{75}$, J. Godfrey ${ }^{142}$, J. Godlewski ${ }^{30}$, M. Goebel ${ }^{42}$, T. Göpfert ${ }^{44}$, C. Goeringer ${ }^{81}$, C. Gössling ${ }^{43}$, S. Goldfarb ${ }^{87}$, T. Golling ${ }^{176}$, A. Gomes ${ }^{124 a, b}$,

L.S. Gomez Fajardo ${ }^{42}$, R. Gonçalo ${ }^{76}$, J. Goncalves Pinto Firmino Da Costa ${ }^{42}$, L. Gonella ${ }^{21}$, S. Gonzalez ${ }^{173}$, S. González de la Hoz ${ }^{167}$, G. Gonzalez Parra ${ }^{12}$, M.L. Gonzalez Silva ${ }^{27}$, S. Gonzalez-Sevilla ${ }^{49}$, J.J. Goodson ${ }^{148}$, L. Goossens ${ }^{30}$, P.A. Gorbounov ${ }^{95}$, H.A. Gordon ${ }^{25}$, I. Gorelov ${ }^{103}$, G. Gorfine ${ }^{175}$, B. Gorini ${ }^{30}$, E. Gorini ${ }^{72 a, 72 b}$, A. Gorišek ${ }^{74}$, E. Gornicki ${ }^{39}$, B. Gosdzik ${ }^{42}$, A.T. Goshaw ${ }^{6}$, M. Gosselink ${ }^{105}$, M.I. Gostkin ${ }^{64}$, I. Gough Eschrich ${ }^{163}$, M. Gouighri ${ }^{135 a}$, D. Goujdami ${ }^{135 c}$, M.P. Goulette ${ }^{49}$, A.G. Goussiou ${ }^{138}$, C. Goy ${ }^{5}$, S. Gozpinar ${ }^{23}$, I. Grabowska-Bold ${ }^{38}$, P. Grafström ${ }^{20 a, 20 b}$, K-J. Grahn ${ }^{42}$, F. Grancagnolo ${ }^{72 a}$, S. Grancagnolo ${ }^{16}$, V. Grassi ${ }^{148}$, V. Gratchev ${ }^{121}$, N. Grau ${ }^{35}$, H.M. Gray ${ }^{30}$, J.A. Gray ${ }^{148}$, E. Graziani134a, O.G. Grebenyuk ${ }^{121}$, T. Greenshaw ${ }^{73}$, Z.D. Greenwood ${ }^{25, m}$, K. Gregersen ${ }^{36}$, I.M. Gregor ${ }^{42}$, P. Grenier ${ }^{143}$, J. Griffiths ${ }^{8}$, N. Grigalashvili ${ }^{64}$, A.A. Grillo ${ }^{137}$, S. Grinstein ${ }^{12}$, Ph. Gris ${ }^{34}$, Y.V. Grishkevich ${ }^{97}$, J.-F. Grivaz ${ }^{115}$, E. Gross ${ }^{172}$, J. Grosse-Knetter ${ }^{54}$, J. Groth-Jensen ${ }^{172}$, K. Grybel ${ }^{141}$, D. Guest ${ }^{176}$, C. Guicheney ${ }^{34}$, S. Guindon ${ }^{54}$, U. Gul ${ }^{53}$, H. Guler ${ }^{85, p}$, J. Gunther ${ }^{125}$, B. Guo ${ }^{158}$, J. Guo ${ }^{35}$, P. Gutierrez ${ }^{111}$, N. Guttman ${ }^{153}$, O. Gutzwiller ${ }^{173}$, C. Guyot ${ }^{136}$, C. Gwenlan ${ }^{118}$, C.B. Gwilliam ${ }^{73}$, A. $\operatorname{Haas}^{143}$, S. $\operatorname{Haas}^{30}$, C. Haber ${ }^{15}$, H.K. Hadavand ${ }^{40}$, D.R. Hadley ${ }^{18}$, P. Haefner ${ }^{21}$, F. Hahn ${ }^{30}$, S. Haider ${ }^{30}$, Z. Hajduk ${ }^{39}$, H. Hakobyan ${ }^{177}$, D. Hall ${ }^{118}$, J. Haller ${ }^{54}$, K. Hamacher ${ }^{175}$, P. Hamal ${ }^{113}$, M. Hamer ${ }^{54}$, A. Hamilton ${ }^{145 b, q}$, S. Hamilton ${ }^{161}$, L. Han ${ }^{33 b}$, K. Hanagaki ${ }^{116}$, K. Hanawa ${ }^{160}$, M. Hance ${ }^{15}$, C. Handel ${ }^{81}$, P. Hanke ${ }^{58 a}$, J.R. Hansen ${ }^{36}$,

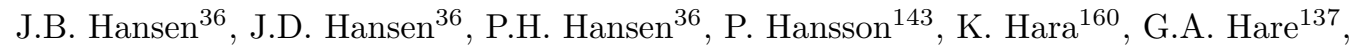
T. Harenberg ${ }^{175}$, S. Harkusha ${ }^{90}$, D. Harper ${ }^{87}$, R.D. Harrington ${ }^{46}$, O.M. Harris ${ }^{138}$, J. Hartert ${ }^{48}$, F. Hartjes ${ }^{105}$, T. Haruyama ${ }^{65}$, A. Harvey ${ }^{56}$, S. Hasegawa ${ }^{101}$, Y. Hasegawa ${ }^{140}$, S. Hassani ${ }^{136}$, S. Haug ${ }^{17}$, M. Hauschild ${ }^{30}$, R. Hauser ${ }^{88}$, M. Havranek ${ }^{21}$, C.M. Hawkes ${ }^{18}$, R.J. Hawkings ${ }^{30}$, A.D. Hawkins ${ }^{79}$, D. Hawkins ${ }^{163}$, T. Hayakawa ${ }^{66}$, T. Hayashi ${ }^{160}$, D. Hayden ${ }^{76}$, C.P. Hays ${ }^{118}$,

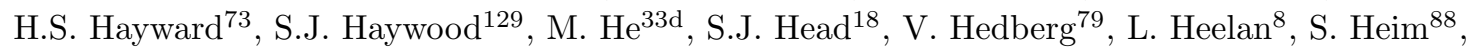
B. Heinemann ${ }^{15}$, S. Heisterkamp ${ }^{36}$, L. Helary ${ }^{22}$, C. Heller ${ }^{98}$, M. Heller ${ }^{30}$, S. Hellman 146a,146b, D. Hellmich ${ }^{21}$, C. Helsens ${ }^{12}$, R.C.W. Henderson ${ }^{71}$, M. Henke ${ }^{58 \mathrm{a}}$, A. Henrichs ${ }^{54}$, A.M. Henriques Correia ${ }^{30}$, S. Henrot-Versille ${ }^{115}$, C. Hensel ${ }^{54}$, T. Henß ${ }^{175}$, C.M. Hernandez ${ }^{8}$, Y. Hernández Jiménez ${ }^{167}$, R. Herrberg ${ }^{16}$, G. Herten ${ }^{48}$, R. Hertenberger ${ }^{98}$, L. Hervas ${ }^{30}$, G.G. Hesketh ${ }^{77}$, N.P. Hessey ${ }^{105}$, E. Higón-Rodriguez ${ }^{167}$, J.C. Hill ${ }^{28}$, K.H. Hiller ${ }^{42}$, S. Hillert ${ }^{21}$, S.J. Hillier ${ }^{18}$, I. Hinchliffe ${ }^{15}$, E. Hines ${ }^{120}$, M. Hirose ${ }^{116}$, F. Hirsch ${ }^{43}$, D. Hirschbuehl ${ }^{175}$, 
J. Hobbs ${ }^{148}$, N. Hod ${ }^{153}$, M.C. Hodgkinson ${ }^{139}$, P. Hodgson ${ }^{139}$, A. Hoecker ${ }^{30}$, M.R. Hoeferkamp ${ }^{103}$, J. Hoffman ${ }^{40}$, D. Hoffmann ${ }^{83}$, M. Hohlfeld ${ }^{81}$, M. Holder ${ }^{141}$, S.O. Holmgren ${ }^{146 a}$, T. Holy ${ }^{127}$, J.L. Holzbauer ${ }^{88}$, T.M. Hong ${ }^{120}$, L. Hooft van Huysduynen ${ }^{108}$, S. Horner ${ }^{48}$, J-Y. Hostachy ${ }^{55}$,

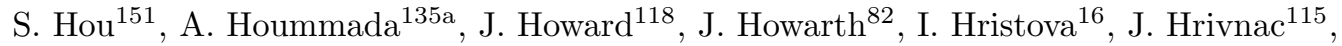
T. Hryn'ova ${ }^{5}$, P.J. $\mathrm{Hsu}^{81}$, S.-C. Hsu ${ }^{15}$, D. $\mathrm{Hu}^{35}$, Z. Hubacek ${ }^{127}$, F. Hubaut ${ }^{83}$, F. Huegging ${ }^{21}$, A. Huettmann ${ }^{42}$, T.B. Huffman ${ }^{118}$, E.W. Hughes ${ }^{35}$, G. Hughes ${ }^{71}$, M. Huhtinen ${ }^{30}$, M. Hurwitz ${ }^{15}$, U. Husemann ${ }^{42}$, N. Huseynov ${ }^{64, r}$, J. Huston ${ }^{88}$, J. $\operatorname{Huth}^{57}$, G. Iacobucci ${ }^{49}$, G. Iakovidis ${ }^{10}$, M. Ibbotson ${ }^{82}$, I. Ibragimov ${ }^{141}$, L. Iconomidou-Fayard ${ }^{115}$, J. Idarraga ${ }^{115}$, P. Iengo ${ }^{102 a}$, O. Igonkina ${ }^{105}$, Y. Ikegami ${ }^{65}$, M. Ikeno ${ }^{65}$, D. Iliadis ${ }^{154}$, N. Ilic $^{158}$, T. Ince ${ }^{21}$, J. Inigo-Golfin ${ }^{30}$, P. Ioannou ${ }^{9}$, M. Iodice ${ }^{134 a}$, K. Iordanidou ${ }^{9}$, V. Ippolito ${ }^{132 a, 132 b}$, A. Irles Quiles ${ }^{167}$, C. Isaksson ${ }^{166}$, M. Ishino ${ }^{67}$, M. Ishitsuka ${ }^{157}$, R. Ishmukhametov ${ }^{40}$, C. Issever ${ }^{118}$, S. Istin ${ }^{19 a}$, A.V. Ivashin ${ }^{128}$, W. Iwanski ${ }^{39}$, H. Iwasaki ${ }^{65}$, J.M. Izen ${ }^{41}$, V. Izzo ${ }^{102 a}$, B. Jackson ${ }^{120}$, J.N. Jackson ${ }^{73}$, P. Jackson ${ }^{1}$, M.R. Jaekel ${ }^{30}$, V. Jain ${ }^{60}$, K. Jakobs ${ }^{48}$, S. Jakobsen ${ }^{36}$, T. Jakoubek ${ }^{125}$, J. Jakubek ${ }^{127}$, D.K. Jana ${ }^{111}$, E. Jansen ${ }^{77}$, H. Jansen ${ }^{30}$, A. Jantsch ${ }^{99}$, M. Janus ${ }^{48}$, G. Jarlskog ${ }^{79}$, L. Jeanty ${ }^{57}$, I. Jen-La Plante ${ }^{31}$, D. Jennens ${ }^{86}$, P. Jenni ${ }^{30}$, A.E. Loevschall-Jensen ${ }^{36}$, P. Jež ${ }^{36}$, S. Jézéquel ${ }^{5}$,

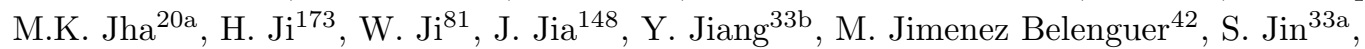
O. Jinnouchi ${ }^{157}$, M.D. Joergensen ${ }^{36}$, D. Joffe ${ }^{40}$, M. Johansen ${ }^{146 a, 146 b}$, K.E. Johansson ${ }^{146 a}$, P. Johansson ${ }^{139}$, S. Johnert ${ }^{42}$, K.A. Johns ${ }^{7}$, K. Jon-And ${ }^{146 a, 146 b}$, G. Jones ${ }^{170}$, R.W.L. Jones ${ }^{71}$, T.J. Jones ${ }^{73}$, C. Joram ${ }^{30}$, P.M. Jorge ${ }^{124 a}$, K.D. Joshi ${ }^{82}$, J. Jovicevic ${ }^{147}$, T. Jovin ${ }^{13 b}$, X. Ju ${ }^{173}$, C.A. Jung ${ }^{43}$, R.M. Jungst ${ }^{30}$, V. Juranek ${ }^{125}$, P. Jussel ${ }^{61}$, A. Juste Rozas ${ }^{12}$, S. Kabana ${ }^{17}$, M. Kaci ${ }^{167}$, A. Kaczmarska ${ }^{39}$, P. Kadlecik ${ }^{36}$, M. Kado ${ }^{115}$, H. Kagan ${ }^{109}$, M. Kagan ${ }^{57}$, E. Kajomovitz ${ }^{152}$, S. Kalinin ${ }^{175}$, L.V. Kalinovskaya ${ }^{64}$, S. Kama ${ }^{40}$, N. Kanaya ${ }^{155}$, M. Kaneda ${ }^{30}$, S. Kaneti ${ }^{28}$, T. Kanno ${ }^{157}$, V.A. Kantserov ${ }^{96}$, J. Kanzaki ${ }^{65}$, B. Kaplan ${ }^{108}$, A. Kapliy ${ }^{31}$, J. Kaplon ${ }^{30}$, D. Kar ${ }^{53}$, M. Karagounis ${ }^{21}$, K. Karakostas ${ }^{10}$, M. Karnevskiy ${ }^{42}$, V. Kartvelishvili ${ }^{71}$, A.N. Karyukhin ${ }^{128}$, L. Kashif ${ }^{173}$, G. Kasieczka ${ }^{58 b}$, R.D. Kass ${ }^{109}$, A. Kastanas $^{14}$, M. Kataoka ${ }^{5}$, Y. Kataoka ${ }^{155}$, E. Katsoufis ${ }^{10}$, J. Katzy ${ }^{42}$, V. Kaushik ${ }^{7}$, K. Kawagoe ${ }^{69}$, T. Kawamoto ${ }^{155}$, G. Kawamura ${ }^{81}$, M.S. Kayl ${ }^{105}$, S. Kazama ${ }^{155}$, V.A. Kazanin ${ }^{107}$, M.Y. Kazarinov ${ }^{64}$, R. Keeler ${ }^{169}$, R. Kehoe ${ }^{40}$, M. Keil ${ }^{54}$, G.D. Kekelidze ${ }^{64}$, J.S. Keller ${ }^{138}$, M. Kenyon ${ }^{53}$, O. Kepka ${ }^{125}$, N. Kerschen ${ }^{30}$, B.P. Kerševan ${ }^{74}$, S. Kersten ${ }^{175}$, K. Kessoku ${ }^{155}$, J. Keung ${ }^{158}$, F. Khalil-zada ${ }^{11}$, H. Khandanyan ${ }^{146 a, 146 b}$, A. Khanov ${ }^{112}$, D. Kharchenko ${ }^{64}$, A. Khodinov ${ }^{96}$, A. Khomich ${ }^{58 a}$, T.J. Khoo ${ }^{28}$, G. Khoriauli ${ }^{21}$, A. Khoroshilov ${ }^{175}$, V. Khovanskiy ${ }^{95}$, E. Khramov ${ }^{64}$, J. Khubua ${ }^{51 b}$, H. Kim ${ }^{146 a, 146 b}$, S.H. Kim ${ }^{160}$, N. Kimura ${ }^{171}$, O. Kind ${ }^{16}$, B.T. King ${ }^{73}$, M. King ${ }^{66}$, R.S.B. King ${ }^{118}$, J. Kirk ${ }^{129}$, A.E. Kiryunin ${ }^{99}$, T. Kishimoto ${ }^{66}$, D. Kisielewska ${ }^{38}$, T. Kitamura ${ }^{66}$, T. Kittelmann ${ }^{123}$, K. Kiuchi ${ }^{160}$, E. Kladiva ${ }^{144 b}$, M. Klein ${ }^{73}$, U. Klein ${ }^{73}$, K. Kleinknecht ${ }^{81}$, M. Klemetti ${ }^{85}$, A. Klier ${ }^{172}$, P. Klimek ${ }^{146 a, 146 b}$, A. Klimentov ${ }^{25}$, R. Klingenberg ${ }^{43}$, J.A. Klinger ${ }^{82}$, E.B. Klinkby ${ }^{36}$, T. Klioutchnikova ${ }^{30}$, P.F. Klok ${ }^{104}$, S. Klous ${ }^{105}$, E.-E. Kluge ${ }^{58 a}$, T. Kluge ${ }^{73}$, P. Kluit ${ }^{105}$, S. Kluth ${ }^{99}$, N.S. Knecht ${ }^{158}$, E. Kneringer ${ }^{61}$, E.B.F.G. Knoops ${ }^{83}$, A. Knue ${ }^{54}$, B.R. Ko ${ }^{45}$, T. Kobayashi ${ }^{155}$, M. Kobel ${ }^{44}$, M. Kocian ${ }^{143}$, P. Kodys ${ }^{126}$, K. Köneke ${ }^{30}$, A.C. König ${ }^{104}$, S. Koenig ${ }^{81}$, L. Köpke ${ }^{81}$, F. Koetsveld ${ }^{104}$, P. Koevesarki ${ }^{21}$, T. Koffas ${ }^{29}$, E. Koffeman ${ }^{105}$, L.A. Kogan ${ }^{118}$, S. Kohlmann ${ }^{175}$, F. Kohn ${ }^{54}$, Z. Kohout ${ }^{127}$, T. Kohriki ${ }^{65}$, T. Koi ${ }^{143}$, G.M. Kolachev ${ }^{107, *}$, H. Kolanoski ${ }^{16}$, V. Kolesnikov ${ }^{64}$, I. Koletsou ${ }^{89 a}$, J. Koll ${ }^{88}$, M. Kollefrath ${ }^{48}$, A.A. Komar ${ }^{94}$, Y. Komori ${ }^{155}$, T. Kondo ${ }^{65}$, T. Kono ${ }^{42, s}$, A.I. Kononov ${ }^{48}$, R. Konoplich ${ }^{108, t}$, N. Konstantinidis ${ }^{77}$, S. Koperny ${ }^{38}$, K. Korcyl ${ }^{39}$, K. Kordas ${ }^{154}$, A. Korn ${ }^{118}$, A. Korol ${ }^{107}$, I. Korolkov ${ }^{12}$, E.V. Korolkova ${ }^{139}$, V.A. Korotkov ${ }^{128}$, O. Kortner ${ }^{99}$, S. Kortner ${ }^{99}$, V.V. Kostyukhin ${ }^{21}$, S. Kotov ${ }^{99}$, V.M. Kotov ${ }^{64}$, A. Kotwal ${ }^{45}$, C. Kourkoumelis ${ }^{9}$, V. Kouskoura ${ }^{154}$, A. Koutsman ${ }^{159 a}$, R. Kowalewski ${ }^{169}$, T.Z. Kowalski ${ }^{38}$, W. Kozanecki ${ }^{136}$, A.S. Kozhin ${ }^{128}$, V. Kral ${ }^{127}$, V.A. Kramarenko ${ }^{97}$, G. Kramberger ${ }^{74}$, M.W. Krasny ${ }^{78}$, A. Krasznahorkay ${ }^{108}$, J.K. Kraus ${ }^{21}$, S. Kreiss ${ }^{108}$, F. Krejci ${ }^{127}$, J. Kretzschmar ${ }^{73}$, N. Krieger ${ }^{54}$, P. Krieger ${ }^{158}$, 


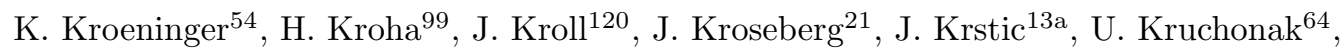
H. Krüger ${ }^{21}$, T. Kruker ${ }^{17}$, N. Krumnack ${ }^{63}$, Z.V. Krumshteyn ${ }^{64}$, T. Kubota ${ }^{86}$, S. Kuday ${ }^{4 a}$, S. Kuehn ${ }^{48}$, A. Kugel ${ }^{58 c}$, T. Kuhl ${ }^{42}$, D. Kuhn ${ }^{61}$, V. Kukhtin ${ }^{64}$, Y. Kulchitsky ${ }^{90}$, S. Kuleshov ${ }^{32 b}$, C. Kummer ${ }^{98}$, M. Kuna ${ }^{78}$, J. Kunkle ${ }^{120}$, A. Kupco ${ }^{125}$, H. Kurashige ${ }^{66}$, M. Kurata ${ }^{160}$, Y.A. Kurochkin ${ }^{90}$, V. Kus ${ }^{125}$, E.S. Kuwertz ${ }^{147}$, M. Kuze ${ }^{157}$, J. Kvita ${ }^{142}$, R. Kwee ${ }^{16}$, A. La Rosa ${ }^{49}$, L. La Rotonda ${ }^{37 a, 37 b}$, L. Labarga ${ }^{80}$, J. Labbe ${ }^{5}$, S. Lablak ${ }^{135 a}$, C. Lacasta ${ }^{167}$, F. Lacava ${ }^{132 a, 132 b}$,

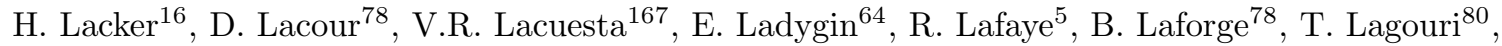
S. Lai ${ }^{48}$, E. Laisne ${ }^{55}$, M. Lamanna ${ }^{30}$, L. Lambourne ${ }^{77}$, C.L. Lampen ${ }^{7}$, W. Lampl ${ }^{7}$, E. Lancon ${ }^{136}$, U. Landgraf ${ }^{48}$, M.P.J. Landon ${ }^{75}$, J.L. Lane ${ }^{82}$, V.S. Lang ${ }^{58 a}$, C. Lange ${ }^{42}$, A.J. Lankford ${ }^{163}$, F. Lanni ${ }^{25}$, K. Lantzsch ${ }^{175}$, S. Laplace ${ }^{78}$, C. Lapoire ${ }^{21}$, J.F. Laporte ${ }^{136}$, T. Lari ${ }^{89 a}$, A. Larner ${ }^{118}$, M. Lassnig ${ }^{30}$, P. Laurelli ${ }^{47}$, V. Lavorini ${ }^{37 a, 37 b}$, W. Lavrijsen ${ }^{15}$, P. Laycock ${ }^{73}$, O. Le Dortz ${ }^{78}$, E. Le Guirriec ${ }^{83}$, C. Le Maner ${ }^{158}$, E. Le Menedeu ${ }^{12}$, T. LeCompte ${ }^{6}$, F. Ledroit-Guillon ${ }^{55}$, H. Lee ${ }^{105}$, J.S.H. Lee ${ }^{116}$, S.C. Lee ${ }^{151}$, L. Lee ${ }^{176}$, M. Lefebvre ${ }^{169}$, M. Legendre ${ }^{136}$, F. Legger ${ }^{98}$, C. Leggett ${ }^{15}$, M. Lehmacher ${ }^{21}$, G. Lehmann Miotto ${ }^{30}$, X. Lei ${ }^{7}$, M.A.L. Leite ${ }^{24 d}$, R. Leitner ${ }^{126}$, D. Lellouch ${ }^{172}$, B. Lemmer ${ }^{54}$, V. Lendermann ${ }^{58 a}$, K.J.C. Leney ${ }^{145 b}$, T. Lenz ${ }^{105}$, G. Lenzen ${ }^{175}$, B. Lenzi ${ }^{30}$, K. Leonhardt ${ }^{44}$, S. Leontsinis ${ }^{10}$, F. Lepold ${ }^{58 a}$, C. Leroy ${ }^{93}$, J-R. Lessard ${ }^{169}$, C.G. Lester ${ }^{28}$, C.M. Lester ${ }^{120}$, J. Levêque ${ }^{5}$, D. Levin ${ }^{87}$, L.J. Levinson ${ }^{172}$, A. Lewis ${ }^{118}$, G.H. Lewis ${ }^{108}$, A.M. Leyko ${ }^{21}$, M. Leyton ${ }^{16}$, B. $\mathrm{Li}^{83}, \mathrm{H}^{2} \mathrm{Li}^{173, u}, \mathrm{~S} . \mathrm{Li}^{33 \mathrm{~b}, v}, \mathrm{X} . \mathrm{Li}^{87}$, Z. Liang ${ }^{118, w}$,

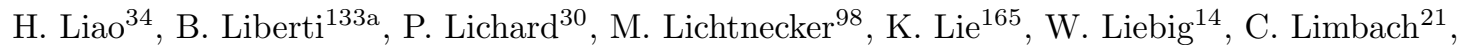
A. Limosani ${ }^{86}$, M. Limper ${ }^{62}$, S.C. $\operatorname{Lin}^{151, x}$, F. Linde ${ }^{105}$, J.T. Linnemann ${ }^{88}$, E. Lipeles ${ }^{120}$, A. Lipniacka ${ }^{14}$, T.M. Liss ${ }^{165}$, D. Lissauer ${ }^{25}$, A. Lister ${ }^{49}$, A.M. Litke ${ }^{137}$, C. Liu ${ }^{29}$, D. Liu ${ }^{151}$,

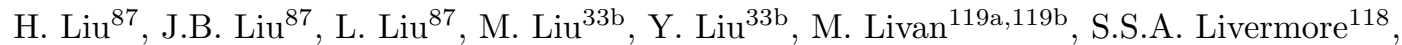
A. Lleres ${ }^{55}$, J. Llorente Merino ${ }^{80}$, S.L. Lloyd ${ }^{75}$, E. Lobodzinska ${ }^{42}$, P. Loch ${ }^{7}$, W.S. Lockman ${ }^{137}$, T. Loddenkoetter ${ }^{21}$, F.K. Loebinger ${ }^{82}$, A. Loginov ${ }^{176}$, C.W. Loh ${ }^{168}$, T. Lohse ${ }^{16}$, K. Lohwasser ${ }^{48}$, M. Lokajicek ${ }^{125}$, V.P. Lombardo ${ }^{5}$, R.E. Long ${ }^{71}$, L. Lopes ${ }^{124 a}$, D. Lopez Mateos ${ }^{57}$, J. Lorenz ${ }^{98}$, N. Lorenzo Martinez ${ }^{115}$, M. Losada ${ }^{162}$, P. Loscutoff ${ }^{15}$, F. Lo Sterzo ${ }^{132 a, 132 b}$, M.J. Losty ${ }^{159 a, *}$, X. Lou ${ }^{41}$, A. Lounis ${ }^{115}$, K.F. Loureiro ${ }^{162}$, J. Love ${ }^{6}$, P.A. Love ${ }^{71}$, A.J. Lowe ${ }^{143, e}$, F. Lu ${ }^{33 a}$, H.J. Lubatti ${ }^{138}$, C. Luci ${ }^{132 a, 132 b}$, A. Lucotte ${ }^{55}$, A. Ludwig ${ }^{44}$, D. Ludwig ${ }^{42}$, I. Ludwig ${ }^{48}$, J. Ludwig ${ }^{48}$, F. Luehring ${ }^{60}$, G. Luijckx ${ }^{105}$, W. Lukas ${ }^{61}$, D. Lumb ${ }^{48}$, L. Luminari ${ }^{132 a}$, E. Lund ${ }^{117}$, B. Lund-Jensen ${ }^{147}$, B. Lundberg ${ }^{79}$, J. Lundberg ${ }^{146 a, 146 b}$, O. Lundberg ${ }^{146 a, 146 b}$, J. Lundquist ${ }^{36}$, M. Lungwitz ${ }^{81}$, D. Lynn ${ }^{25}$, E. Lytken ${ }^{79}$, H. Ma ${ }^{25}$, L.L. Ma ${ }^{173}$, G. Maccarrone ${ }^{47}$, A. Macchiolo ${ }^{99}$, B. Maček ${ }^{74}$, J. Machado Miguens ${ }^{124 a}$, R. Mackeprang ${ }^{36}$, R.J. Madaras ${ }^{15}$, H.J. Maddocks ${ }^{71}$, W.F. Mader ${ }^{44}$, R. Maennerr ${ }^{58 c}$, T. Maeno ${ }^{25}$, P. Mättig ${ }^{175}$, S. Mättig ${ }^{81}$, L. Magnoni ${ }^{163}$, E. Magradze ${ }^{54}$, K. Mahboubi ${ }^{48}$, S. Mahmoud ${ }^{73}$, G. Mahout ${ }^{18}$, C. Maiani ${ }^{136}$, C. Maidantchik ${ }^{24 a}$, A. Maio ${ }^{124 a}, b$, S. Majewski ${ }^{25}$, Y. Makida ${ }^{65}$, N. Makovec ${ }^{115}$, P. Mal ${ }^{136}$, B. Malaescu ${ }^{30}$, Pa. Malecki ${ }^{39}$, P. Malecki ${ }^{39}$, V.P. Maleev ${ }^{121}$, F. Malek ${ }^{55}$, U. Mallik ${ }^{62}$, D. Malon ${ }^{6}$, C. Malone ${ }^{143}$, S. Maltezos ${ }^{10}$, V. Malyshev ${ }^{107}$, S. Malyukov ${ }^{30}$, R. Mameghani ${ }^{98}$, J. Mamuzic ${ }^{13 b}$, A. Manabe ${ }^{65}$, L. Mandelli ${ }^{89 a}$, I. Mandić ${ }^{74}$, R. Mandrysch ${ }^{16}$, J. Maneira ${ }^{124 a}$, A. Manfredini ${ }^{99}$, P.S. Mangeard ${ }^{88}$, L. Manhaes de Andrade Filho ${ }^{24 b}$, J.A. Manjarres Ramos ${ }^{136}$, A. Mann ${ }^{54}$, P.M. Manning ${ }^{137}$, A. Manousakis-Katsikakis ${ }^{9}$, B. Mansoulie ${ }^{136}$, A. Mapelli ${ }^{30}$, L. Mapelli ${ }^{30}$, L. March $^{80}$, J.F. Marchand ${ }^{29}$, F. Marchese ${ }^{133 a, 133 b}$, G. Marchiori ${ }^{78}$, M. Marcisovsky ${ }^{125}$, C.P. Marino ${ }^{169}$, F. Marroquim ${ }^{24 a}$, Z. Marshall ${ }^{30}$, F.K. Martens ${ }^{158}$, L.F. Marti ${ }^{17}$, S. Marti-Garcia ${ }^{167}$, B. Martin ${ }^{30}$, B. Martin ${ }^{88}$, J.P. Martin ${ }^{93}$, T.A. Martin ${ }^{18}$, V.J. Martin ${ }^{46}$, B. Martin dit Latour ${ }^{49}$, S. Martin-Haugh ${ }^{149}$, M. Martinez ${ }^{12}$, V. Martinez Outschoorn ${ }^{57}$, A.C. Martyniuk ${ }^{169}$, M. Marx ${ }^{82}$, F. Marzano ${ }^{132 a}$, A. Marzin ${ }^{111}$, L. Masetti ${ }^{81}$, T. Mashimo ${ }^{155}$, R. Mashinistov ${ }^{94}$, J. Masik $^{82}$, A.L. Maslennikov ${ }^{107}$, I. Massa ${ }^{20 a, 20 b}$, G. Massaro ${ }^{105}$, N. Massol $^{5}$, P. Mastrandrea ${ }^{148}$, A. Mastroberardino ${ }^{37 a, 37 b}$, T. Masubuchi ${ }^{155}$, P. Matricon ${ }^{115}$, H. Matsunaga ${ }^{155}$, T. Matsushita ${ }^{66}$, C. Mattravers ${ }^{118, c}$, J. Maurer ${ }^{83}$, S.J. Maxfield ${ }^{73}$, A. Mayne ${ }^{139}$, R. Mazini ${ }^{151}$, M. Mazur ${ }^{21}$, 
L. Mazzaferro ${ }^{133 a, 133 b}$, M. Mazzanti ${ }^{89 a}$, J. Mc Donald ${ }^{85}$, S.P. Mc Kee ${ }^{87}$, A. McCarn ${ }^{165}$, R.L. McCarthy ${ }^{148}$, T.G. McCarthy ${ }^{29}$, N.A. McCubbin ${ }^{129}$, K.W. McFarlane ${ }^{56, *}$, J.A. Mcfayden ${ }^{139}$, G. Mchedlidze ${ }^{51 b}$, T. Mclaughlan ${ }^{18}$, S.J. McMahon ${ }^{129}$, R.A. McPherson ${ }^{169, k}$, A. Meade ${ }^{84}$, J. Mechnich ${ }^{105}$, M. Mechtel ${ }^{175}$, M. Medinnis ${ }^{42}$, R. Meera-Lebbai ${ }^{111}$, T. Meguro ${ }^{116}$, R. Mehdiyev ${ }^{93}$, S. Mehlhase ${ }^{36}$, A. Mehta ${ }^{73}$, K. Meier ${ }^{58 a}$, B. Meirose ${ }^{79}$, C. Melachrinos ${ }^{31}$, B.R. Mellado Garcia ${ }^{173}$, F. Meloni ${ }^{89 a, 89 b}$, L. Mendoza Navas ${ }^{162}$, Z. Meng ${ }^{151, u}$, A. Mengarelli ${ }^{20 a, 20 b}$, S. Menke ${ }^{99}$, E. Meoni ${ }^{161}$, K.M. Mercurio 57 , P. Mermod ${ }^{49}$, L. Merola ${ }^{102 a, 102 b}$, C. Meroni ${ }^{89 a}$, F.S. Merritt ${ }^{31}$, H. Merritt ${ }^{109}$, A. Messina ${ }^{30, y}$, J. Metcalfe ${ }^{25}$, A.S. Mete ${ }^{163}$, C. Meyer ${ }^{81}$, C. Meyer ${ }^{31}$, J-P. Meyer ${ }^{136}$, J. Meyer ${ }^{174}$, J. Meyer ${ }^{54}$, T.C. Meyer $^{30}$, J. Miao ${ }^{33 d}$, S. Michal ${ }^{30}$, L. Micu ${ }^{26 a}$, R.P. Middleton ${ }^{129}$, S. Migas $^{73}$, L. Mijović ${ }^{136}$, G. Mikenberg ${ }^{172}$, M. Mikestikova ${ }^{125}$, M. Mikuž ${ }^{74}$, D.W. Miller ${ }^{31}$, R.J. Miller ${ }^{88}$, W.J. Mills ${ }^{168}$, C. Mills ${ }^{57}$, A. Milov ${ }^{172}$, D.A. Milstead ${ }^{146 a, 146 b}$, D. Milstein ${ }^{172}$, A.A. Minaenko ${ }^{128}$, M. Miñano Moya ${ }^{167}$, I.A. Minashvili ${ }^{64}$, A.I. Mincer ${ }^{108}$, B. Mindur ${ }^{38}$, M. Mineev ${ }^{64}$, Y. Ming ${ }^{173}$, L.M. Mir ${ }^{12}$, G. Mirabelli ${ }^{132 a}$, J. Mitrevski137, V.A. Mitsou ${ }^{167}$, S. Mitsui ${ }^{65}$, P.S. Miyagawa ${ }^{139}$, J.U. Mjörnmark ${ }^{79}$, T. Moa ${ }^{146 a, 146 b}$, V. Moeller ${ }^{28}$, K. Mönig ${ }^{42}$, N. Möser ${ }^{21}$, S. Mohapatra ${ }^{148}$, W. Mohr ${ }^{48}$, R. Moles-Valls ${ }^{167}$, J. Monk ${ }^{77}$, E. Monnier ${ }^{83}$, J. Montejo Berlingen ${ }^{12}$, F. Monticelli ${ }^{70}$, S. Monzani ${ }^{20 a, 20 b}$, R.W. Moore ${ }^{3}$, G.F. Moorhead ${ }^{86}$, C. Mora Herrera ${ }^{49}$, A. Moraes ${ }^{53}$, N. Morange ${ }^{136}$, J. Morel ${ }^{54}$, G. Morello $37 \mathrm{a}, 37 \mathrm{~b}$, D. Moreno ${ }^{81}$, M. Moreno Llácer ${ }^{167}$, P. Morettini ${ }^{50 a}$, M. Morgenstern ${ }^{44}$, M. Morii ${ }^{57}$, A.K. Morley ${ }^{30}$, G. Mornacchi ${ }^{30}$, J.D. Morris ${ }^{75}$, L. Morvaj ${ }^{101}$, H.G. Moser ${ }^{99}$, M. Mosidze ${ }^{51 b}$, J. Moss ${ }^{109}$, R. Mount ${ }^{143}$, E. Mountricha ${ }^{10, z}$, S.V. Mouraviev ${ }^{94, *}$, E.J.W. Moyse ${ }^{84}$, F. Mueller $^{58 a}$, J. Mueller ${ }^{123}$, K. Mueller ${ }^{21}$, T.A. Müller ${ }^{98}$, T. Mueller ${ }^{81}$, D. Muenstermann ${ }^{30}$, Y. Munwes ${ }^{153}$, W.J. Murray ${ }^{129}$, I. Mussche ${ }^{105}$, E. Musto ${ }^{102 a, 102 b}$, A.G. Myagkov ${ }^{128}$, M. Myska ${ }^{125}$, J. Nadal $^{12}$, K. Nagai ${ }^{160}$, R. Nagai ${ }^{157}$, K. Nagano ${ }^{65}$, A. Nagarkar ${ }^{109}$, Y. Nagasaka ${ }^{59}$, M. Nagel ${ }^{99}$, A.M. Nairz ${ }^{30}$, Y. Nakahama ${ }^{30}$, K. Nakamura ${ }^{155}$, T. Nakamura ${ }^{155}$, I. Nakano ${ }^{110}$, G. Nanava ${ }^{21}$, A. Napier ${ }^{161}$, R. Narayan ${ }^{58 b}$, M. Nash ${ }^{77, c}$, T. Nattermann ${ }^{21}$, T. Naumann ${ }^{42}$, G. Navarro ${ }^{162}$, H.A. Neal ${ }^{87}$, P.Yu. Nechaeva ${ }^{94}$, T.J. Neep ${ }^{82}$, A. Negri ${ }^{119 a, 119 b}$, G. Negri ${ }^{30}$, M. Negrini ${ }^{20 a}$, S. Nektarijevic ${ }^{49}$, A. Nelson ${ }^{163}$, T.K. Nelson ${ }^{143}$, S. Nemecek ${ }^{125}$, P. Nemethy ${ }^{108}$, A.A. Nepomuceno ${ }^{24 a}$, M. Nessi ${ }^{30, a a}$, M.S. Neubauer ${ }^{165}$, M. Neumann ${ }^{175}$, A. Neusiedl ${ }^{81}$, R.M. Neves ${ }^{108}$, P. Nevski ${ }^{25}$, P.R. Newman ${ }^{18}$, V. Nguyen Thi Hong ${ }^{136}$, R.B. Nickerson ${ }^{118}$, R. Nicolaidou ${ }^{136}$, B. Nicquevert ${ }^{30}$, F. Niedercorn ${ }^{115}$, J. Nielsen ${ }^{137}$, N. Nikiforou ${ }^{35}$, A. Nikiforov ${ }^{16}$, V. Nikolaenko ${ }^{128}$, I. Nikolic-Audit ${ }^{78}$, K. Nikolics ${ }^{49}$, K. Nikolopoulos ${ }^{18}$, H. Nilsen ${ }^{48}$, P. Nilsson ${ }^{8}$, Y. Ninomiya ${ }^{155}$, A. Nisati ${ }^{132 a}$, R. Nisius ${ }^{99}$, T. Nobe ${ }^{157}$, L. Nodulman ${ }^{6}$, M. Nomachi ${ }^{116}$, I. Nomidis ${ }^{154}$, S. Norberg ${ }^{111}$, M. Nordberg ${ }^{30}$, P.R. Norton ${ }^{129}$, J. Novakova ${ }^{126}$, M. Nozaki ${ }^{65}$, L. Nozka ${ }^{113}$, I.M. Nugent ${ }^{159 a}$, A.-E. Nuncio-Quiroz ${ }^{21}$, G. Nunes Hanninger ${ }^{86}$, T. Nunnemann ${ }^{98}$, E. Nurse ${ }^{77}$, B.J. O'Brien ${ }^{46}$, S.W. O’Neale ${ }^{18, *}$, D.C. O’Neil ${ }^{142}$, V. O'Shea ${ }^{53}$, L.B. Oakes ${ }^{98}$,

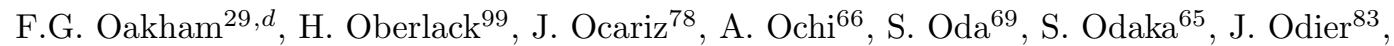
H. Ogren ${ }^{60}$, A. $\mathrm{Oh}^{82}$, S.H. Oh ${ }^{45}$, C.C. Ohm ${ }^{30}$, T. Ohshima ${ }^{101}$, H. Okawa ${ }^{25}$, Y. Okumura ${ }^{31}$, T. Okuyama ${ }^{155}$, A. Olariu ${ }^{26 a}$, A.G. Olchevski64 ${ }^{64}$ S.A. Olivares Pino ${ }^{32 a}$, M. Oliveira ${ }^{124 a}, h$, D. Oliveira Damazio ${ }^{25}$, E. Oliver Garcia ${ }^{167}$, D. Olivito ${ }^{120}$, A. Olszewski ${ }^{39}$, J. Olszowska ${ }^{39}$, A. Onofre ${ }^{124 a, a b}$, P.U.E. Onyisi ${ }^{31}$, C.J. Oram ${ }^{159 a}$, M.J. Oreglia ${ }^{31}$, Y. Oren ${ }^{153}$,

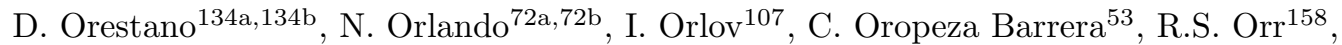
B. Osculati ${ }^{50 a, 50 b}$, R. Ospanov ${ }^{120}$, C. Osuna ${ }^{12}$, G. Otero y Garzon ${ }^{27}$, J.P. Ottersbach ${ }^{105}$, M. Ouchrif ${ }^{135 d}$, E.A. Ouellette ${ }^{169}$, F. Ould-Saada ${ }^{117}$, A. Ouraou ${ }^{136}$, Q. Ouyang ${ }^{33 a}$,

A. Ovcharova ${ }^{15}$, M. Owen ${ }^{82}$, S. Owen ${ }^{139}$, V.E. Ozcan ${ }^{19 a}$, N. Ozturk ${ }^{8}$, A. Pacheco Pages ${ }^{12}$, C. Padilla Aranda ${ }^{12}$, S. Pagan Griso ${ }^{15}$, E. Paganis ${ }^{139}$, C. Pahl ${ }^{99}$, F. Paige ${ }^{25}$, P. Pais ${ }^{84}$, K. Pajchel ${ }^{117}$, G. Palacino ${ }^{159 b}$, C.P. Paleari ${ }^{7}$, S. Palestini $^{30}$, D. Pallin ${ }^{34}$, A. Palma ${ }^{124 a}$, J.D. Palmer ${ }^{18}$, Y.B. Pan $^{173}$, E. Panagiotopoulou ${ }^{10}$, P. Pani ${ }^{105}$, N. Panikashvili ${ }^{87}$, S. Panitkin ${ }^{25}$, D. Pantea ${ }^{26 a}$, A. Papadelis ${ }^{146 a}$, Th.D. Papadopoulou ${ }^{10}$, A. Paramonov ${ }^{6}$, D. Paredes Hernandez ${ }^{34}$, 
W. Park ${ }^{25, a c}$, M.A. Parker ${ }^{28}$, F. Parodi ${ }^{50 a, 50 b}$, J.A. Parsons ${ }^{35}$, U. Parzefall ${ }^{48}$, S. Pashapour $^{54}$, E. Pasqualucci ${ }^{132 a}$, S. Passaggio ${ }^{50 a}$, A. Passeri ${ }^{134 a}$, F. Pastore ${ }^{134 a, 134 b, *}$, Fr. Pastore ${ }^{76}$,

G. Pásztor ${ }^{49, a d}$, S. Pataraia ${ }^{175}$, N. Patel ${ }^{150}$, J.R. Pater ${ }^{82}$, S. Patricelli102a,102b ${ }^{10}$ T. Pauly ${ }^{30}$,

M. Pecsy ${ }^{144 a}$, S. Pedraza Lopez ${ }^{167}$, M.I. Pedraza Morales ${ }^{173}$, S.V. Peleganchuk ${ }^{107}$, D. Pelikan ${ }^{166}$,

H. Peng ${ }^{33 b}$, B. Penning ${ }^{31}$, A. Penson ${ }^{35}$, J. Penwell ${ }^{60}$, M. Perantoni ${ }^{24 a}$, K. Perez ${ }^{35, a e}$,

T. Perez Cavalcanti ${ }^{42}$, E. Perez Codina ${ }^{159 a}$, M.T. Pérez García-Estañ ${ }^{167}$, V. Perez Reale ${ }^{35}$,

L. Perini ${ }^{89 a, 89 b}$, H. Pernegger ${ }^{30}$, R. Perrino ${ }^{72 a}$, P. Perrodo ${ }^{5}$, V.D. Peshekhonov ${ }^{64}$, K. Peters ${ }^{30}$,

B.A. Petersen ${ }^{30}$, J. Petersen ${ }^{30}$, T.C. Petersen ${ }^{36}$, E. Petit ${ }^{5}$, A. Petridis ${ }^{154}$, C. Petridou ${ }^{154}$,

E. Petrolo ${ }^{132 a}$, F. Petrucci ${ }^{134 a, 134 b}$, D. Petschull ${ }^{42}$, M. Petteni ${ }^{142}$, R. Pezoa ${ }^{32 b}$, A. Phan ${ }^{86}$,

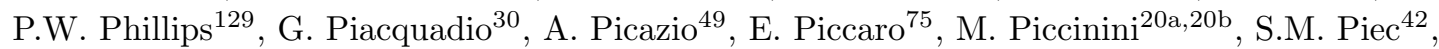

R. Piegaia ${ }^{27}$, D.T. Pignotti ${ }^{109}$, J.E. Pilcher ${ }^{31}$, A.D. Pilkington ${ }^{82}$, J. Pina ${ }^{124 a, b}$,

M. Pinamonti164a,164c , A. Pinder ${ }^{118}$, J.L. Pinfold ${ }^{3}$, B. Pinto ${ }^{124 a}$, C. Pizio ${ }^{89 a, 89 b}$,

M. Plamondon ${ }^{169}$, M.-A. Pleier ${ }^{25}$, E. Plotnikova ${ }^{64}$, A. Poblaguev ${ }^{25}$, S. Poddar $^{58 a}$, F. Podlyski ${ }^{34}$,

L. Poggioli ${ }^{115}$, D. Pohl ${ }^{21}$, M. Pohl ${ }^{49}$, G. Polesello ${ }^{119 a}$, A. Policicchio ${ }^{37 a, 37 b}$, A. Polini ${ }^{20 a}$, J. Poll $^{75}$,

V. Polychronakos ${ }^{25}$, D. Pomeroy ${ }^{23}$, K. Pommès ${ }^{30}$, L. Pontecorvo ${ }^{132 a}$, B.G. Pope ${ }^{88}$,

G.A. Popeneciu ${ }^{26 a}$, D.S. Popovic ${ }^{13 a}$, A. Poppleton ${ }^{30}$, X. Portell Bueso ${ }^{30}$, G.E. Pospelov ${ }^{99}$,

S. Pospisil ${ }^{127}$, I.N. Potrap ${ }^{99}$, C.J. Potter ${ }^{149}$, C.T. Potter ${ }^{114}$, G. Poulard ${ }^{30}$, J. Poveda ${ }^{60}$,

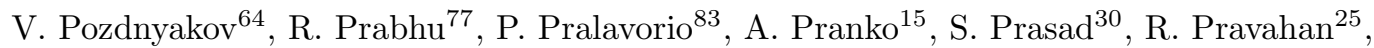

S. Prell ${ }^{63}$, K. Pretzl ${ }^{17}$, D. Price ${ }^{60}$, J. Price ${ }^{73}$, L.E. Price ${ }^{6}$, D. Prieur ${ }^{123}$, M. Primavera ${ }^{72 a}$,

K. Prokofiev ${ }^{108}$, F. Prokoshin ${ }^{32 b}$, S. Protopopescu ${ }^{25}$, J. Proudfoot ${ }^{6}$, X. Prudent ${ }^{44}$,

M. Przybycien ${ }^{38}$, H. Przysiezniak ${ }^{5}$, S. Psoroulas ${ }^{21}$, E. Ptacek ${ }^{114}$, E. Pueschel ${ }^{84}$, J. Purdham ${ }^{87}$,

M. Purohit ${ }^{25, a c}$, P. Puzo ${ }^{115}$, Y. Pylypchenko ${ }^{62}$, J. Qian ${ }^{87}$, A. Quadt ${ }^{54}$, D.R. Quarrie ${ }^{15}$,

W.B. Quayle ${ }^{173}$, F. Quinonez ${ }^{32 a}$, M. $\operatorname{Raas}^{104}$, V. Radescu ${ }^{42}$, P. Radloff ${ }^{114}$, T. Rador ${ }^{19 a}$,

F. Ragusa ${ }^{89 a, 89 b}$, G. Rahal ${ }^{178}$, A.M. Rahimi ${ }^{109}$, D. Rahm ${ }^{25}$, S. Rajagopalan ${ }^{25}$, M. Rammensee ${ }^{48}$,

M. Rammes ${ }^{141}$, A.S. Randle-Conde ${ }^{40}$, K. Randrianarivony ${ }^{29}$, F. Rauscher ${ }^{98}$, T.C. Rave ${ }^{48}$,

M. Raymond ${ }^{30}$, A.L. Read ${ }^{117}$, D.M. Rebuzzi ${ }^{119 a, 119 b}$, A. Redelbach ${ }^{174}$, G. Redlinger ${ }^{25}$,

R. Reece ${ }^{120}$, K. Reeves ${ }^{41}$, E. Reinherz-Aronis ${ }^{153}$, A. Reinsch ${ }^{114}$, I. Reisinger ${ }^{43}$, C. Rembser ${ }^{30}$,

Z.L. Ren ${ }^{151}$, A. Renaud ${ }^{115}$, M. Rescigno ${ }^{132 a}$, S. Resconi ${ }^{89 a}$, B. Resende ${ }^{136}$, P. Reznicek $^{98}$,

R. Rezvani ${ }^{158}$, R. Richter ${ }^{99}$, E. Richter-Was ${ }^{5}$ af, M. Ridel ${ }^{78}$, M. Rijpstra ${ }^{105}$, M. Rijssenbeek ${ }^{148}$,

A. Rimoldi ${ }^{119 a, 119 b}$, L. Rinaldi ${ }^{20 a}$, R.R. $\operatorname{Rios}^{40}$, I. Riu ${ }^{12}$, G. Rivoltella ${ }^{89 a, 89 b}$, F. Rizatdinova ${ }^{112}$,

E. Rizvi ${ }^{75}$, S.H. Robertson ${ }^{85, k}$, A. Robichaud-Veronneau ${ }^{118}$, D. Robinson ${ }^{28}$, J.E.M. Robinson ${ }^{82}$,

A. Robson ${ }^{53}$, J.G. Rocha de Lima ${ }^{106}$, C. Roda ${ }^{122 a, 122 b}$, D. Roda Dos Santos ${ }^{30}$, A. Roe ${ }^{54}$,

S. Roe ${ }^{30}$, O. Røhne ${ }^{117}$, S. Rolli ${ }^{161}$, A. Romaniouk ${ }^{96}$, M. Romano ${ }^{20 a, 20 b}$, G. Romeo ${ }^{27}$,

E. Romero Adam ${ }^{167}$, N. Rompotis ${ }^{138}$, L. Roos ${ }^{78}$, E. Ros ${ }^{167}$, S. Rosati ${ }^{132 a}$, K. Rosbach ${ }^{49}$,

A. Rose $^{149}$, M. Rose ${ }^{76}$, G.A. Rosenbaum ${ }^{158}$, E.I. Rosenberg ${ }^{63}$, P.L. Rosendahl ${ }^{14}$, O. Rosenthal ${ }^{141}$,

L. Rosselet ${ }^{49}$, V. Rossetti ${ }^{12}$, E. Rossi ${ }^{132 a, 132 b}$, L.P. Rossi ${ }^{50 a}$, M. Rotaru ${ }^{26 a}$, I. Roth ${ }^{172}$,

J. Rothberg ${ }^{138}$, D. Rousseau ${ }^{115}$, C.R. Royon ${ }^{136}$, A. Rozanov ${ }^{83}$, Y. Rozen ${ }^{152}$, X. Ruan ${ }^{33 a, a g}$,

F. Rubbo ${ }^{12}$, I. Rubinskiy ${ }^{42}$, N. Ruckstuhl ${ }^{105}$, V.I. Rud ${ }^{97}$, C. Rudolph ${ }^{44}$, G. Rudolph ${ }^{61}$, F. Rühr $^{7}$,

A. Ruiz-Martinez ${ }^{63}$, L. Rumyantsev ${ }^{64}$, Z. Rurikova ${ }^{48}$, N.A. Rusakovich ${ }^{64}$, J.P. Rutherfoord ${ }^{7}$,

C. Ruwiedel ${ }^{15, *}$, P. Ruzicka ${ }^{125}$, Y.F. Ryabov ${ }^{121}$, M. Rybar ${ }^{126}$, G. Rybkin ${ }^{115}$, N.C. Ryder ${ }^{118}$,

A.F. Saavedra ${ }^{150}$, I. Sadeh ${ }^{153}$, H.F-W. Sadrozinski ${ }^{137}$, R. Sadykov ${ }^{64}$, F. Safai Tehrani ${ }^{132 a}$,

H. Sakamoto ${ }^{155}$, G. Salamanna ${ }^{75}$, A. Salamon ${ }^{133 a}$, M. Saleem ${ }^{111}$, D. Salek ${ }^{30}$, D. Salihagic ${ }^{99}$,

A. Salnikov ${ }^{143}$, J. Salt ${ }^{167}$, B.M. Salvachua Ferrando ${ }^{6}$, D. Salvatore ${ }^{37 a, 37 b}$, F. Salvatore ${ }^{149}$,

A. Salvucci ${ }^{104}$, A. Salzburger ${ }^{30}$, D. Sampsonidis ${ }^{154}$, B.H. Samset ${ }^{117}$, A. Sanchez ${ }^{102 a, 102 b}$,

V. Sanchez Martinez ${ }^{167}$, H. Sandaker ${ }^{14}$, H.G. Sander ${ }^{81}$, M.P. Sanders ${ }^{98}$, M. Sandhoff ${ }^{175}$,

T. Sandoval ${ }^{28}$, C. Sandoval ${ }^{162}$, R. Sandstroem ${ }^{99}$, D.P.C. Sankey ${ }^{129}$, A. Sansoni ${ }^{47}$,

C. Santamarina Rios ${ }^{85}$, C. Santoni ${ }^{34}$, R. Santonico ${ }^{133 a, 133 b}$, H. Santos ${ }^{124 a}$, J.G. Saraiva ${ }^{124 a}$,

T. Sarangi ${ }^{173}$, E. Sarkisyan-Grinbaum ${ }^{8}$, F. Sarri ${ }^{122 a, 122 b}$, G. Sartisohn ${ }^{175}$, O. Sasaki ${ }^{65}$, 
Y. Sasaki ${ }^{155}$, N. Sasao ${ }^{67}$, I. Satsounkevitch ${ }^{90}$, G. Sauvage ${ }^{5, *}$, E. Sauvan $^{5}$, J.B. Sauvan ${ }^{115}$, P. Savard ${ }^{158, d}$, V. Savinov ${ }^{123}$, D.O. Savu $^{30}$, L. Sawyer ${ }^{25, m}$, D.H. Saxon ${ }^{53}$, J. Saxon ${ }^{120}$,

C. Sbarra ${ }^{20 a}$, A. Sbrizzi ${ }^{20 a, 20 b}$, D.A. Scannicchio ${ }^{163}$, M. Scarcella ${ }^{150}$, J. Schaarschmidt ${ }^{115}$,

P. Schacht ${ }^{99}$, D. Schaefer ${ }^{120}$, U. Schäfer ${ }^{81}$, S. Schaepe ${ }^{21}$, S. Schaetzel ${ }^{58 b}$, A.C. Schaffer ${ }^{115}$,

D. Schaile ${ }^{98}$, R.D. Schamberger ${ }^{148}$, A.G. Schamov ${ }^{107}$, V. Scharf ${ }^{58 a}$, V.A. Schegelsky ${ }^{121}$,

D. Scheirich ${ }^{87}$, M. Schernau ${ }^{163}$, M.I. Scherzer ${ }^{35}$, C. Schiavi ${ }^{50 a, 50 b}$, J. Schieck ${ }^{98}$,

M. Schioppa ${ }^{37 a, 37 b}$, S. Schlenker ${ }^{30}$, E. Schmidt ${ }^{48}$, K. Schmieden ${ }^{21}$, C. Schmitt ${ }^{81}$, S. Schmitt ${ }^{58 b}$,

M. Schmitz ${ }^{21}$, B. Schneider ${ }^{17}$, U. Schnoor ${ }^{44}$, A. Schoening ${ }^{58 b}$, A.L.S. Schorlemmer ${ }^{54}$, M. Schott ${ }^{30}$,

D. Schouten ${ }^{159 a}$, J. Schovancova ${ }^{125}$, M. Schram ${ }^{85}$, C. Schroeder ${ }^{81}$, N. Schroer ${ }^{58 c}$,

M.J. Schultens ${ }^{21}$, J. Schultes ${ }^{175}$, H.-C. Schultz-Coulon ${ }^{58 a}$, H. Schulz ${ }^{16}$, M. Schumacher ${ }^{48}$,

B.A. Schumm ${ }^{137}$, Ph. Schune ${ }^{136}$, C. Schwanenberger ${ }^{82}$, A. Schwartzman ${ }^{143}$, Ph. Schwegler ${ }^{99}$,

Ph. Schwemling ${ }^{78}$, R. Schwienhorst ${ }^{88}$, R. Schwierz ${ }^{44}$, J. Schwindling ${ }^{136}$, T. Schwindt ${ }^{21}$,

M. Schwoerer ${ }^{5}$, G. Sciolla ${ }^{23}$, W.G. Scott ${ }^{129}$, J. Searcy ${ }^{114}$, G. Sedov ${ }^{42}$, E. Sedykh ${ }^{121}$,

S.C. Seidel ${ }^{103}$, A. Seiden ${ }^{137}$, F. Seifert ${ }^{44}$, J.M. Seixas ${ }^{24 a}$, G. Sekhniaidze ${ }^{102 a}$, S.J. Sekula ${ }^{40}$,

K.E. Selbach ${ }^{46}$, D.M. Seliverstov ${ }^{121}$, B. Sellden ${ }^{146 a}$, G. Sellers ${ }^{73}$, M. Seman ${ }^{144 b}$,

N. Semprini-Cesari ${ }^{20 a, 20 b}$, C. Serfon ${ }^{98}$, L. Serin ${ }^{115}$, L. Serkin ${ }^{54}$, R. Seuster ${ }^{99}$, H. Severini ${ }^{111}$,

A. Sfyrla ${ }^{30}$, E. Shabalina ${ }^{54}$, M. Shamim ${ }^{114}$, L.Y. Shan ${ }^{33 a}$, J.T. Shank ${ }^{22}$, Q.T. Shao ${ }^{86}$,

M. Shapiro ${ }^{15}$, P.B. Shatalov ${ }^{95}$, K. Shaw ${ }^{164 a, 164 c}$, D. Sherman ${ }^{176}$, P. Sherwood ${ }^{77}$, A. Shibata ${ }^{108}$,

S. Shimizu ${ }^{101}$, M. Shimojima ${ }^{100}$, T. Shin ${ }^{56}$, M. Shiyakova ${ }^{64}$, A. Shmeleva ${ }^{94}$, M.J. Shochet ${ }^{31}$,

D. Short ${ }^{118}$, S. Shrestha ${ }^{63}$, E. Shulga ${ }^{96}$, M.A. Shupe ${ }^{7}$, P. Sicho ${ }^{125}$, A. Sidoti ${ }^{132 a}$, F. Siegert ${ }^{48}$,

Dj. Sijacki ${ }^{13 a}$, O. Silbert ${ }^{172}$, J. Silva ${ }^{124 a}$, Y. Silver ${ }^{153}$, D. Silverstein ${ }^{143}$, S.B. Silverstein ${ }^{146 a}$,

V. Simak ${ }^{127}$, O. Simard ${ }^{136}$, Lj. Simic ${ }^{13 a}$, S. Simion ${ }^{115}$, E. Simioni ${ }^{81}$, B. Simmons ${ }^{77}$,

R. Simoniello ${ }^{89 a, 89 b}$, M. Simonyan ${ }^{36}$, P. Sinervo ${ }^{158}$, N.B. Sinev ${ }^{114}$, V. Sipica ${ }^{141}$, G. Siragusa ${ }^{174}$,

A. Sircar $^{25}$, A.N. Sisakyan ${ }^{64, *}$, S.Yu. Sivoklokov ${ }^{97}$, J. Sjölin ${ }^{146 a, 146 b}$, T.B. Sjursen ${ }^{14}$,

L.A. Skinnari ${ }^{15}$, H.P. Skottowe ${ }^{57}$, K. Skovpen ${ }^{107}$, P. Skubic ${ }^{111}$, M. Slater ${ }^{18}$, T. Slavicek ${ }^{127}$,

K. Sliwa ${ }^{161}$, V. Smakhtin ${ }^{172}$, B.H. Smart ${ }^{46}$, S.Yu. Smirnov ${ }^{96}$, Y. Smirnov ${ }^{96}$, L.N. Smirnova ${ }^{97}$,

O. Smirnova ${ }^{79}$, B.C. Smith ${ }^{57}$, D. Smith ${ }^{143}$, K.M. Smith ${ }^{53}$, M. Smizanska ${ }^{71}$, K. Smolek ${ }^{127}$,

A.A. Snesarev ${ }^{94}$, S.W. Snow ${ }^{82}$, J. Snow ${ }^{111}$, S. Snyder ${ }^{25}$, R. Sobie ${ }^{169, k}$, J. Sodomka ${ }^{127}$,

A. Soffer ${ }^{153}$, C.A. Solans ${ }^{167}$, M. Solar ${ }^{127}$, J. Solc ${ }^{127}$, E.Yu. Soldatov ${ }^{96}$, U. Soldevila ${ }^{167}$,

E. Solfaroli Camillocci ${ }^{132 a, 132 b}$, A.A. Solodkov ${ }^{128}$, O.V. Solovyanov ${ }^{128}$, V. Solovyev ${ }^{121}$, N. Soni ${ }^{1}$,

V. Sopko ${ }^{127}$, B. Sopko ${ }^{127}$, M. Sosebee ${ }^{8}$, R. Soualah ${ }^{164 a, 164 c}$, A. Soukharev ${ }^{107}$, S. Spagnolo ${ }^{72 a, 72 b}$,

F. Spanò ${ }^{76}$, R. Spighi ${ }^{20 a}$, G. Spigo ${ }^{30}$, R. Spiwoks ${ }^{30}$, M. Spousta ${ }^{126, a h}$, T. Spreitzer ${ }^{158}$,

B. Spurlock ${ }^{8}$, R.D. St. Denis ${ }^{53}$, J. Stahlman ${ }^{120}$, R. Stamen ${ }^{58 a}$, E. Stanecka ${ }^{39}$, R.W. Stanek ${ }^{6}$,

C. Stanescu ${ }^{134 a}$, M. Stanescu-Bellu ${ }^{42}$, S. Stapnes ${ }^{117}$, E.A. Starchenko ${ }^{128}$, J. Stark ${ }^{55}$,

P. Staroba ${ }^{125}$, P. Starovoitov ${ }^{42}$, R. Staszewski ${ }^{39}$, A. Staude ${ }^{98}$, P. Stavina ${ }^{144 a, *}$, G. Steele ${ }^{53}$,

P. Steinbach ${ }^{44}$, P. Steinberg ${ }^{25}$, I. Stekl ${ }^{127}$, B. Stelzer ${ }^{142}$, H.J. Stelzer ${ }^{88}$, O. Stelzer-Chilton ${ }^{159 a}$,

H. Stenzel ${ }^{52}$, S. Stern ${ }^{99}$, G.A. Stewart ${ }^{30}$, J.A. Stillings ${ }^{21}$, M.C. Stockton ${ }^{85}$, K. Stoerig ${ }^{48}$,

G. Stoicea ${ }^{26 a}$, S. Stonjek ${ }^{99}$, P. Strachota ${ }^{126}$, A.R. Stradling ${ }^{8}$, A. Straessner ${ }^{44}$, J. Strandberg ${ }^{147}$,

S. Strandberg 146a,146b, A. Strandlie ${ }^{117}$, M. Strang ${ }^{109}$, E. Strauss ${ }^{143}$, M. Strauss ${ }^{111}$,

P. Strizenec ${ }^{144 b}$, R. Ströhmer ${ }^{174}$, D.M. Strom ${ }^{114}$, J.A. Strong ${ }^{76, *}$, R. Stroynowski ${ }^{40}$, J. Strube ${ }^{129}$,

B. Stugu ${ }^{14}$, I. Stumer ${ }^{25, *}$, J. Stupak ${ }^{148}$, P. Sturm ${ }^{175}$, N.A. Styles ${ }^{42}$, D.A. Soh ${ }^{151, w}$, D. Su ${ }^{143}$,

HS. Subramania ${ }^{3}$, A. Succurro ${ }^{12}$, Y. Sugaya ${ }^{116}$, C. Suhr ${ }^{106}$, M. Suk ${ }^{126}$, V.V. Sulin ${ }^{94}$,

S. Sultansoy ${ }^{4 d}$, T. Sumida ${ }^{67}$, X. Sun ${ }^{55}$, J.E. Sundermann ${ }^{48}$, K. Suruliz ${ }^{139}$, G. Susinno ${ }^{37 a, 37 b}$,

M.R. Sutton ${ }^{149}$, Y. Suzuki ${ }^{65}$, Y. Suzuki ${ }^{66}$, M. Svatos ${ }^{125}$, S. Swedish ${ }^{168}$, I. Sykora ${ }^{144 a}$,

T. Sykora ${ }^{126}$, J. Sánchez ${ }^{167}$, D. Ta ${ }^{105}$, K. Tackmann ${ }^{42}$, A. Taffard ${ }^{163}$, R. Tafirout ${ }^{159 a}$,

N. Taiblum ${ }^{153}$, Y. Takahashi ${ }^{101}$, H. Takai ${ }^{25}$, R. Takashima ${ }^{68}$, H. Takeda ${ }^{66}$, T. Takeshita ${ }^{140}$,

Y. Takubo ${ }^{65}$, M. Talby ${ }^{83}$, A. Talyshev ${ }^{107, f}$, M.C. Tamsett ${ }^{25}$, J. Tanaka ${ }^{155}$, R. Tanaka ${ }^{115}$,

S. Tanaka ${ }^{131}$, S. Tanaka ${ }^{65}$, A.J. Tanasijczuk ${ }^{142}$, K. Tani ${ }^{66}$, N. Tannoury ${ }^{83}$, S. Tapprogge ${ }^{81}$, 
D. Tardif ${ }^{158}$, S. Tarem ${ }^{152}$, F. Tarrade ${ }^{29}$, G.F. Tartarelli ${ }^{89 a}$, P. Tas ${ }^{126}$, M. Tasevsky ${ }^{125}$, E. Tassi ${ }^{37 a, 37 b}$, M. Tatarkhanov ${ }^{15}$, Y. Tayalati ${ }^{135 d}$, C. Taylor ${ }^{77}$, F.E. Taylor ${ }^{92}$, G.N. Taylor ${ }^{86}$, W. Taylor ${ }^{159 b}$, M. Teinturier ${ }^{115}$, F.A. Teischinger ${ }^{30}$, M. Teixeira Dias Castanheira ${ }^{75}$, P. Teixeira-Dias ${ }^{76}$, K.K. Temming ${ }^{48}$, H. Ten Kate ${ }^{30}$, P.K. Teng ${ }^{151}$, S. Terada ${ }^{65}$, K. Terashi ${ }^{155}$, J. Terron ${ }^{80}$, M. Testa ${ }^{47}$, R.J. Teuscher ${ }^{158, k}$, J. Therhaag ${ }^{21}$, T. Theveneaux-Pelzer ${ }^{78}$, S. Thoma ${ }^{48}$, J.P. Thomas $^{18}$, E.N. Thompson ${ }^{35}$, P.D. Thompson ${ }^{18}$, P.D. Thompson ${ }^{158}$, A.S. Thompson ${ }^{53}$, L.A. Thomsen ${ }^{36}$, E. Thomson ${ }^{120}$, M. Thomson ${ }^{28}$, W.M. Thong ${ }^{86}$, R.P. Thun ${ }^{87}$, F. Tian ${ }^{35}$, M.J. Tibbetts ${ }^{15}$, T. Tic ${ }^{125}$, V.O. Tikhomirov ${ }^{94}$, Y.A. Tikhonov ${ }^{107, f}$, S. Timoshenko ${ }^{96}$, P. Tipton ${ }^{176}$, S. Tisserant ${ }^{83}$, T. Todorov ${ }^{5}$, S. Todorova-Nova ${ }^{161}$, B. Toggerson ${ }^{163}$, J. Tojo ${ }^{69}$,

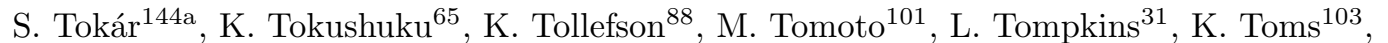
A. Tonoyan $^{14}$, C. Topfel ${ }^{17}$, N.D. Topilin ${ }^{64}$, I. Torchiani ${ }^{30}$, E. Torrence ${ }^{114}$, H. Torres ${ }^{78}$, E. Torró

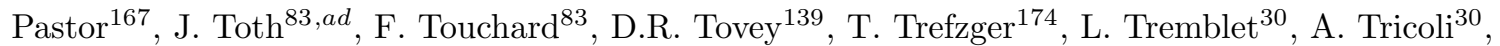
I.M. Trigger ${ }^{159 a}$, S. Trincaz-Duvoid ${ }^{78}$, M.F. Tripiana ${ }^{70}$, N. Triplett ${ }^{25}$, W. Trischuk ${ }^{158}$,

B. Trocmé ${ }^{55}$, C. Troncon ${ }^{89 a}$, M. Trottier-McDonald ${ }^{142}$, M. Trzebinski ${ }^{39}$, A. Trzupek ${ }^{39}$, C. Tsarouchas $^{30}$, J.C-L. Tseng ${ }^{118}$, M. Tsiakiris ${ }^{105}$, P.V. Tsiareshka ${ }^{90}$, D. Tsionou ${ }^{5, a i}$,

G. Tsipolitis ${ }^{10}$, S. Tsiskaridze ${ }^{12}$, V. Tsiskaridze ${ }^{48}$, E.G. Tskhadadze ${ }^{51 a}$, I.I. Tsukerman ${ }^{95}$, V. Tsulaia ${ }^{15}$, J.-W. Tsung ${ }^{21}$, S. Tsuno ${ }^{65}$, D. Tsybychev ${ }^{148}$, A. Tua ${ }^{139}$, A. Tudorache ${ }^{26 a}$, V. Tudorache ${ }^{26 a}$, J.M. Tuggle ${ }^{31}$, M. Turala ${ }^{39}$, D. Turecek ${ }^{127}$, I. Turk Cakir ${ }^{4}$, E. Turlay ${ }^{105}$,

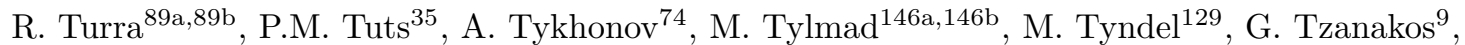

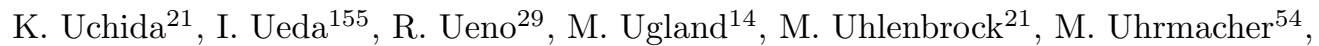

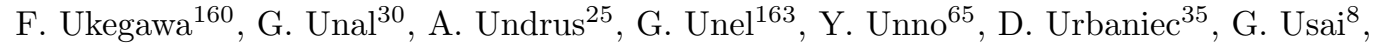
M. Uslenghi ${ }^{119 a, 119 b}$, L. Vacavant ${ }^{83}$, V. Vacek ${ }^{127}$, B. Vachon ${ }^{85}$, S. Vahsen ${ }^{15}$, J. Valenta ${ }^{125}$,

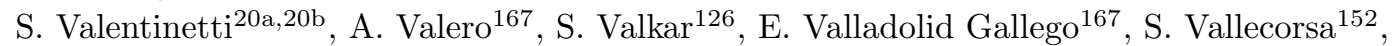
J.A. Valls Ferrer ${ }^{167}$, P.C. Van Der Deijl ${ }^{105}$, R. van der Geer $^{105}$, H. van der Graaf ${ }^{105}$, R. Van Der Leeuw ${ }^{105}$, E. van der Poel $^{105}$, D. van der Ster $^{30}$, N. van Eldik ${ }^{30}$, P. van Gemmeren ${ }^{6}$, I. van Vulpen ${ }^{105}$, M. Vanadia ${ }^{99}$, W. Vandelli ${ }^{30}$, A. Vaniachine ${ }^{6}$, P. Vankov ${ }^{42}$, F. Vannucci ${ }^{78}$, R. Vari ${ }^{132 a}$, T. Varol $^{84}$, D. Varouchas ${ }^{15}$, A. Vartapetian ${ }^{8}$, K.E. Varvell ${ }^{150}$, V.I. Vassilakopoulos ${ }^{56}$, F. Vazeille ${ }^{34}$, T. Vazquez Schroeder ${ }^{54}$, G. Vegni ${ }^{89 a, 89 b}$, J.J. Veillet ${ }^{115}$, F. Veloso ${ }^{124 a}$, R. Veness ${ }^{30}$,

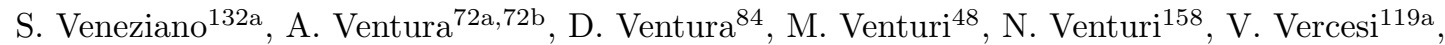
M. Verducci ${ }^{138}$, W. Verkerke ${ }^{105}$, J.C. Vermeulen ${ }^{105}$, A. Vest ${ }^{44}$, M.C. Vetterli ${ }^{142, d}$, I. Vichou ${ }^{165}$, T. Vickey ${ }^{145 b, a j}$, O.E. Vickey Boeriu ${ }^{145 b}$, G.H.A. Viehhauser ${ }^{118}$, S. Viel $^{168}$, M. Villa ${ }^{20 a, 20 b}$, M. Villaplana Perez ${ }^{167}$, E. Vilucchi ${ }^{47}$, M.G. Vincter ${ }^{29}$, E. Vinek ${ }^{30}$, V.B. Vinogradov ${ }^{64}$, M. Virchaux ${ }^{136, *}$, J. Virzi ${ }^{15}$, O. Vitells ${ }^{172}$, M. Viti ${ }^{42}$, I. Vivarelli ${ }^{48}$, F. Vives Vaque ${ }^{3}$, S. Vlachos $^{10}$,

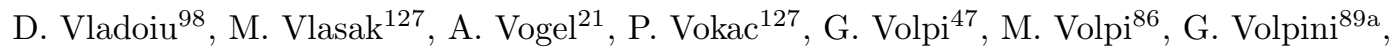
H. von der Schmitt ${ }^{99}$, H. von Radziewski ${ }^{48}$, E. von Toerne ${ }^{21}$, V. Vorobel ${ }^{126}$, V. Vorwerk ${ }^{12}$,

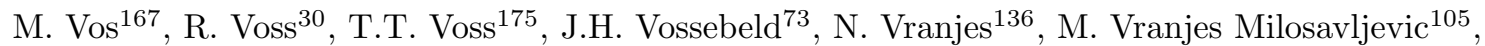
V. Vrba ${ }^{125}$, M. Vreeswijk ${ }^{105}$, T. Vu Anh ${ }^{48}$, R. Vuillermet ${ }^{30}$, I. Vukotic ${ }^{31}$, W. Wagner ${ }^{175}$, P. Wagner ${ }^{120}$, H. Wahlen ${ }^{175}$, S. Wahrmund ${ }^{44}$, J. Wakabayashi ${ }^{101}$, S. Walch ${ }^{87}$, J. Walder ${ }^{71}$, R. Walker ${ }^{98}$, W. Walkowiak ${ }^{141}$, R. Wall ${ }^{176}$, P. Waller ${ }^{73}$, B. Walsh ${ }^{176}$, C. Wang ${ }^{45}$, H. Wang ${ }^{173}$,

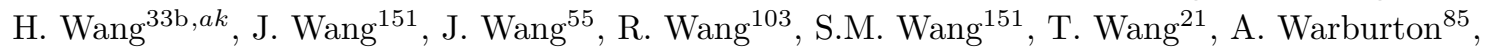
C.P. Ward ${ }^{28}$, M. Warsinsky ${ }^{48}$, A. Washbrook ${ }^{46}$, C. Wasicki ${ }^{42}$, I. Watanabe ${ }^{66}$, P.M. Watkins ${ }^{18}$, A.T. Watson ${ }^{18}$, I.J. Watson ${ }^{150}$, M.F. Watson ${ }^{18}$, G. Watts ${ }^{138}$, S. Watts ${ }^{82}$, A.T. Waugh ${ }^{150}$, B.M. Waugh ${ }^{77}$, M.S. Weber ${ }^{17}$, P. Weber ${ }^{54}$, A.R. Weidberg ${ }^{118}$, P. Weigell ${ }^{99}$, J. Weingarten ${ }^{54}$, C. Weiser ${ }^{48}$, H. Wellenstein ${ }^{23}$, P.S. Wells ${ }^{30}$, T. Wenaus ${ }^{25}$, D. Wendland ${ }^{16}$, Z. Weng ${ }^{151, w}$, T. Wengler ${ }^{30}$, S. Wenig ${ }^{30}$, N. Wermes ${ }^{21}$, M. Werner ${ }^{48}$, P. Werner ${ }^{30}$, M. Werth $^{163}$, M. Wessels ${ }^{58 a}$, J. Wetter ${ }^{161}$, C. Weydert ${ }^{55}$, K. Whalen ${ }^{29}$, S.J. Wheeler-Ellis ${ }^{163}$, A. White ${ }^{8}$, M.J. White ${ }^{86}$, S. White ${ }^{122 a, 122 b}$, S.R. Whitehead ${ }^{118}$, D. Whiteson ${ }^{163}$, D. Whittington ${ }^{60}$, F. Wicek ${ }^{115}$, D. Wicke ${ }^{175}$, F.J. Wickens ${ }^{129}$, W. Wiedenmann ${ }^{173}$, M. Wielers ${ }^{129}$, P. Wienemann ${ }^{21}$, 
C. Wiglesworth ${ }^{75}$, L.A.M. Wiik-Fuchs ${ }^{48}$, P.A. Wijeratne ${ }^{77}$, A. Wildauer ${ }^{99}$, M.A. Wildt ${ }^{42, s}$, I. Wilhelm ${ }^{126}$, H.G. Wilkens ${ }^{30}$, J.Z. Will ${ }^{98}$, E. Williams ${ }^{35}$, H.H. Williams ${ }^{120}$, W. Willis ${ }^{35}$, S. Willocq ${ }^{84}$, J.A. Wilson ${ }^{18}$, M.G. Wilson ${ }^{143}$, A. Wilson ${ }^{87}$, I. Wingerter-Seez ${ }^{5}$, S. Winkelmann ${ }^{48}$, F. Winklmeier ${ }^{30}$, M. Wittgen ${ }^{143}$, S.J. Wollstadt ${ }^{81}$, M.W. Wolter ${ }^{39}$, H. Wolters ${ }^{124 a, h}$, W.C. Wong ${ }^{41}$, G. Wooden ${ }^{87}$, B.K. Wosiek ${ }^{39}$, J. Wotschack ${ }^{30}$, M.J. Woudstra ${ }^{82}$, K.W. Wozniak ${ }^{39}$, K. Wraight ${ }^{53}$, M. Wright ${ }^{53}$, B. Wrona ${ }^{73}$, S.L. Wu ${ }^{173}, \mathrm{X} . \mathrm{Wu}^{49}, \mathrm{Y} . \mathrm{Wu}^{33 \mathrm{~b}, a l}$, E. Wulf ${ }^{35}$, B.M. Wynne ${ }^{46}$, S. Xella ${ }^{36}$, M. Xiao ${ }^{136}$, S. Xie ${ }^{48}$, C. Xu ${ }^{33 b, z}$, D. Xu ${ }^{139}$, B. Yabsley ${ }^{150}$, S. Yacoob ${ }^{145 a, a m}$, M. Yamada ${ }^{65}$, H. Yamaguchi ${ }^{155}$, A. Yamamoto ${ }^{65}$, K. Yamamoto ${ }^{63}$, S. Yamamoto ${ }^{155}$, T. Yamamura ${ }^{155}$, T. Yamanaka ${ }^{155}$, J. Yamaoka ${ }^{45}$, T. Yamazaki ${ }^{155}$, Y. Yamazaki ${ }^{66}$, Z. Yan ${ }^{22}$, H. Yang ${ }^{87}$, U.K. Yang ${ }^{82}$, Y. Yang ${ }^{60}$, Z. Yang ${ }^{146,146 b}$, S. Yanush ${ }^{91}$, L. Yao ${ }^{33 a}$, Y. Yao ${ }^{15}$, Y. Yasu ${ }^{65}$, G.V. Ybeles Smit ${ }^{130}$, J. Ye ${ }^{40}$, S. Ye ${ }^{25}$, M. Yilmaz ${ }^{4 c}$,

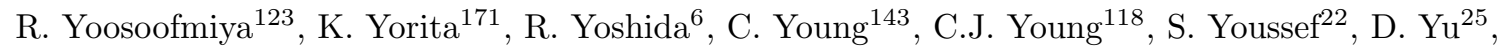
J. Yu ${ }^{8}$, J. Yu ${ }^{112}$, L. Yuan ${ }^{66}$, A. Yurkewicz ${ }^{106}$, M. Byszewski ${ }^{30}$, B. Zabinski ${ }^{39}$, R. Zaidan ${ }^{62}$, A.M. Zaitsev ${ }^{128}$, Z. Zajacova ${ }^{30}$, L. Zanello ${ }^{132 a, 132 b}$, D. Zanzi ${ }^{99}$, A. Zaytsev ${ }^{25}$, C. Zeitnitz ${ }^{175}$, M. Zeman ${ }^{125}$, A. Zemla ${ }^{39}$, C. Zendler ${ }^{21}$, O. Zenin ${ }^{128}$, T. Ženiš ${ }^{144 a}$, Z. Zinonos ${ }^{122 a, 122 b}$, S. Zenz ${ }^{15}$, D. Zerwas ${ }^{115}$, G. Zevi della Porta ${ }^{57}$, Z. Zhan ${ }^{33 d}$, D. Zhang ${ }^{33 b, a k}$, H. Zhang ${ }^{88}$, J. Zhang ${ }^{6}$, X. Zhang ${ }^{33 d}$, Z. Zhang ${ }^{115}$, L. Zhao ${ }^{108}$, T. Zhao ${ }^{138}$, Z. Zhao ${ }^{33 b}$, A. Zhemchugov ${ }^{64}$, J. Zhong ${ }^{118}$, B. Zhou ${ }^{87}$, N. Zhou ${ }^{163}$, Y. Zhou ${ }^{151}$, C.G. Zhu ${ }^{33 d}$, H. Zhu ${ }^{42}$, J. Zhu ${ }^{87}$, Y. Zhu ${ }^{33 b}$, X. Zhuang ${ }^{98}$, V. Zhuravlov ${ }^{99}$, D. Zieminska ${ }^{60}$, N.I. Zimin ${ }^{64}$, R. Zimmermann ${ }^{21}$, S. Zimmermann ${ }^{21}$, S. Zimmermann ${ }^{48}$, M. Ziolkowski ${ }^{141}$, R. Zitoun ${ }^{5}$, L. Živković ${ }^{35}$, V.V. Zmouchko ${ }^{128, *}$, G. Zobernig ${ }^{173}$, A. Zoccoli ${ }^{20 a, 20 b}$, M. zur Nedden ${ }^{16}$, V. Zutshi ${ }^{106}$, L. Zwalinski ${ }^{30}$.

${ }^{1}$ School of Chemistry and Physics, University of Adelaide, North Terrace Campus, 5000, SA, Australia

2 Physics Department, SUNY Albany, Albany NY, United States of America

3 Department of Physics, University of Alberta, Edmonton AB, Canada

4 (a) Department of Physics, Ankara University, Ankara; ${ }^{(b)}$ Department of Physics, Dumlupinar University, Kutahya; ${ }^{(c)}$ Department of Physics, Gazi University, Ankara; ${ }^{(d)}$ Division of Physics, TOBB University of Economics and Technology, Ankara; ${ }^{(e)}$ Turkish Atomic Energy Authority, Ankara, Turkey

${ }^{5}$ LAPP, CNRS/IN2P3 and Université de Savoie, Annecy-le-Vieux, France

${ }^{6}$ High Energy Physics Division, Argonne National Laboratory, Argonne IL, United States of America

7 Department of Physics, University of Arizona, Tucson AZ, United States of America

8 Department of Physics, The University of Texas at Arlington, Arlington TX, United States of America

9 Physics Department, University of Athens, Athens, Greece

${ }^{10}$ Physics Department, National Technical University of Athens, Zografou, Greece

11 Institute of Physics, Azerbaijan Academy of Sciences, Baku, Azerbaijan

${ }^{12}$ Institut de Física d'Altes Energies and Departament de Física de la Universitat Autònoma de Barcelona and ICREA, Barcelona, Spain

$13{ }^{(a)}$ Institute of Physics, University of Belgrade, Belgrade; ${ }^{(b)}$ Vinca Institute of Nuclear Sciences, University of Belgrade, Belgrade, Serbia

14 Department for Physics and Technology, University of Bergen, Bergen, Norway

${ }^{15}$ Physics Division, Lawrence Berkeley National Laboratory and University of California, Berkeley CA, United States of America

${ }^{16}$ Department of Physics, Humboldt University, Berlin, Germany

17 Albert Einstein Center for Fundamental Physics and Laboratory for High Energy Physics, University of Bern, Bern, Switzerland

18 School of Physics and Astronomy, University of Birmingham, Birmingham, United Kingdom

$19{ }^{(a)}$ Department of Physics, Bogazici University, Istanbul; ${ }^{(b)}$ Division of Physics, Dogus University, Istanbul; ${ }^{(c)}$ Department of Physics Engineering, Gaziantep University, Gaziantep; ${ }^{(d)}$ Department of Physics, Istanbul Technical University, Istanbul, Turkey

$20{ }^{(a)}$ INFN Sezione di Bologna; ${ }^{(b)}$ Dipartimento di Fisica, Università di Bologna, Bologna, Italy

${ }^{21}$ Physikalisches Institut, University of Bonn, Bonn, Germany 
22 Department of Physics, Boston University, Boston MA, United States of America

23 Department of Physics, Brandeis University, Waltham MA, United States of America

24 (a) Universidade Federal do Rio De Janeiro COPPE/EE/IF, Rio de Janeiro; ${ }^{(b)}$ Federal University of Juiz de Fora (UFJF), Juiz de Fora; ${ }^{\left({ }^{(c)}\right.}$ Federal University of Sao Joao del Rei (UFSJ), Sao Joao del Rei;

${ }^{(d)}$ Instituto de Fisica, Universidade de Sao Paulo, Sao Paulo, Brazil

25 Physics Department, Brookhaven National Laboratory, Upton NY, United States of America

$26{ }^{(a)}$ National Institute of Physics and Nuclear Engineering, Bucharest; ${ }^{(b)}$ University Politehnica Bucharest, Bucharest; ${ }^{(c)}$ West University in Timisoara, Timisoara, Romania

${ }^{27}$ Departamento de Física, Universidad de Buenos Aires, Buenos Aires, Argentina

${ }^{28}$ Cavendish Laboratory, University of Cambridge, Cambridge, United Kingdom

29 Department of Physics, Carleton University, Ottawa ON, Canada

30 CERN, Geneva, Switzerland

31 Enrico Fermi Institute, University of Chicago, Chicago IL, United States of America

$32{ }^{(a)}$ Departamento de Física, Pontificia Universidad Católica de Chile, Santiago; ${ }^{(b)}$ Departamento de Física, Universidad Técnica Federico Santa María, Valparaíso, Chile

33 (a) Institute of High Energy Physics, Chinese Academy of Sciences, Beijing; ${ }^{(b)}$ Department of Modern Physics, University of Science and Technology of China, Anhui; ${ }^{\left({ }^{c}\right)}$ Department of Physics, Nanjing University, Jiangsu; ${ }^{(d)}$ School of Physics, Shandong University, Shandong, China

${ }^{34}$ Laboratoire de Physique Corpusculaire, Clermont Université and Université Blaise Pascal and CNRS/IN2P3, Aubiere Cedex, France

${ }^{35}$ Nevis Laboratory, Columbia University, Irvington NY, United States of America

36 Niels Bohr Institute, University of Copenhagen, Kobenhavn, Denmark

37 (a) INFN Gruppo Collegato di Cosenza; ${ }^{(b)}$ Dipartimento di Fisica, Università della Calabria, Arcavata di Rende, Italy

38 AGH University of Science and Technology, Faculty of Physics and Applied Computer Science, Krakow, Poland

39 The Henryk Niewodniczanski Institute of Nuclear Physics, Polish Academy of Sciences, Krakow, Poland

40 Physics Department, Southern Methodist University, Dallas TX, United States of America

${ }^{41}$ Physics Department, University of Texas at Dallas, Richardson TX, United States of America

42 DESY, Hamburg and Zeuthen, Germany

43 Institut für Experimentelle Physik IV, Technische Universität Dortmund, Dortmund, Germany

${ }^{4}$ Institut für Kern-und Teilchenphysik, Technical University Dresden, Dresden, Germany

${ }^{4}$ Department of Physics, Duke University, Durham NC, United States of America

${ }^{46}$ SUPA - School of Physics and Astronomy, University of Edinburgh, Edinburgh, United Kingdom

47 INFN Laboratori Nazionali di Frascati, Frascati, Italy

48 Fakultät für Mathematik und Physik, Albert-Ludwigs-Universität, Freiburg, Germany

49 Section de Physique, Université de Genève, Geneva, Switzerland

$50{ }^{(a)}$ INFN Sezione di Genova; ${ }^{(b)}$ Dipartimento di Fisica, Università di Genova, Genova, Italy

$51{ }^{(a)}$ E. Andronikashvili Institute of Physics, Tbilisi State University, Tbilisi; ${ }^{(b)}$ High Energy Physics Institute, Tbilisi State University, Tbilisi, Georgia

52 II Physikalisches Institut, Justus-Liebig-Universität Giessen, Giessen, Germany

53 SUPA - School of Physics and Astronomy, University of Glasgow, Glasgow, United Kingdom

54 II Physikalisches Institut, Georg-August-Universität, Göttingen, Germany

${ }^{55}$ Laboratoire de Physique Subatomique et de Cosmologie, Université Joseph Fourier and CNRS/IN2P3 and Institut National Polytechnique de Grenoble, Grenoble, France

56 Department of Physics, Hampton University, Hampton VA, United States of America

57 Laboratory for Particle Physics and Cosmology, Harvard University, Cambridge MA, United States of America

58 (a) Kirchhoff-Institut für Physik, Ruprecht-Karls-Universität Heidelberg, Heidelberg; ${ }^{(b)}$ Physikalisches Institut, Ruprecht-Karls-Universität Heidelberg, Heidelberg; ${ }^{(c)}$ ZITI Institut für technische Informatik, Ruprecht-Karls-Universität Heidelberg, Mannheim, Germany

${ }^{59}$ Faculty of Applied Information Science, Hiroshima Institute of Technology, Hiroshima, Japan 
60 Department of Physics, Indiana University, Bloomington IN, United States of America

${ }^{61}$ Institut für Astro-und Teilchenphysik, Leopold-Franzens-Universität, Innsbruck, Austria

${ }^{62}$ University of Iowa, Iowa City IA, United States of America

63 Department of Physics and Astronomy, Iowa State University, Ames IA, United States of America

64 Joint Institute for Nuclear Research, JINR Dubna, Dubna, Russia

${ }^{65}$ KEK, High Energy Accelerator Research Organization, Tsukuba, Japan

${ }^{66}$ Graduate School of Science, Kobe University, Kobe, Japan

${ }^{67}$ Faculty of Science, Kyoto University, Kyoto, Japan

${ }^{68}$ Kyoto University of Education, Kyoto, Japan

69 Department of Physics, Kyushu University, Fukuoka, Japan

70 Instituto de Física La Plata, Universidad Nacional de La Plata and CONICET, La Plata, Argentina

71 Physics Department, Lancaster University, Lancaster, United Kingdom

$72{ }^{(a)}$ INFN Sezione di Lecce; ${ }^{(b)}$ Dipartimento di Matematica e Fisica, Università del Salento, Lecce, Italy

73 Oliver Lodge Laboratory, University of Liverpool, Liverpool, United Kingdom

74 Department of Physics, Jožef Stefan Institute and University of Ljubljana, Ljubljana, Slovenia

75 School of Physics and Astronomy, Queen Mary University of London, London, United Kingdom

${ }^{76}$ Department of Physics, Royal Holloway University of London, Surrey, United Kingdom

77 Department of Physics and Astronomy, University College London, London, United Kingdom

${ }^{78}$ Laboratoire de Physique Nucléaire et de Hautes Energies, UPMC and Université Paris-Diderot and CNRS/IN2P3, Paris, France

${ }^{79}$ Fysiska institutionen, Lunds universitet, Lund, Sweden

80 Departamento de Fisica Teorica C-15, Universidad Autonoma de Madrid, Madrid, Spain

81 Institut für Physik, Universität Mainz, Mainz, Germany

82 School of Physics and Astronomy, University of Manchester, Manchester, United Kingdom

${ }^{83}$ CPPM, Aix-Marseille Université and CNRS/IN2P3, Marseille, France

84 Department of Physics, University of Massachusetts, Amherst MA, United States of America

${ }^{85}$ Department of Physics, McGill University, Montreal QC, Canada

86 School of Physics, University of Melbourne, Victoria, Australia

87 Department of Physics, The University of Michigan, Ann Arbor MI, United States of America

${ }^{88}$ Department of Physics and Astronomy, Michigan State University, East Lansing MI, United States of America

$89{ }^{(a)}$ INFN Sezione di Milano; ${ }^{(b)}$ Dipartimento di Fisica, Università di Milano, Milano, Italy

90 B.I. Stepanov Institute of Physics, National Academy of Sciences of Belarus, Minsk, Republic of Belarus

91 National Scientific and Educational Centre for Particle and High Energy Physics, Minsk, Republic of Belarus

${ }^{92}$ Department of Physics, Massachusetts Institute of Technology, Cambridge MA, United States of America

${ }^{93}$ Group of Particle Physics, University of Montreal, Montreal QC, Canada

94 P.N. Lebedev Institute of Physics, Academy of Sciences, Moscow, Russia

${ }^{95}$ Institute for Theoretical and Experimental Physics (ITEP), Moscow, Russia

${ }_{96}$ Moscow Engineering and Physics Institute (MEPhI), Moscow, Russia

97 Skobeltsyn Institute of Nuclear Physics, Lomonosov Moscow State University, Moscow, Russia

${ }^{98}$ Fakultät für Physik, Ludwig-Maximilians-Universität München, München, Germany

99 Max-Planck-Institut für Physik (Werner-Heisenberg-Institut), München, Germany

100 Nagasaki Institute of Applied Science, Nagasaki, Japan

101 Graduate School of Science and Kobayashi-Maskawa Institute, Nagoya University, Nagoya, Japan

$102{ }^{(a)}$ INFN Sezione di Napoli; ${ }^{(b)}$ Dipartimento di Scienze Fisiche, Università di Napoli, Napoli, Italy

103 Department of Physics and Astronomy, University of New Mexico, Albuquerque NM, United States of America

104 Institute for Mathematics, Astrophysics and Particle Physics, Radboud University Nijmegen/Nikhef, Nijmegen, Netherlands

105 Nikhef National Institute for Subatomic Physics and University of Amsterdam, Amsterdam, Netherlands

106 Department of Physics, Northern Illinois University, DeKalb IL, United States of America 
107 Budker Institute of Nuclear Physics, SB RAS, Novosibirsk, Russia

108 Department of Physics, New York University, New York NY, United States of America

109 Ohio State University, Columbus OH, United States of America

${ }^{110}$ Faculty of Science, Okayama University, Okayama, Japan

111 Homer L. Dodge Department of Physics and Astronomy, University of Oklahoma, Norman OK, United States of America

112 Department of Physics, Oklahoma State University, Stillwater OK, United States of America

113 Palacký University, RCPTM, Olomouc, Czech Republic

114 Center for High Energy Physics, University of Oregon, Eugene OR, United States of America

115 LAL, Université Paris-Sud and CNRS/IN2P3, Orsay, France

116 Graduate School of Science, Osaka University, Osaka, Japan

117 Department of Physics, University of Oslo, Oslo, Norway

118 Department of Physics, Oxford University, Oxford, United Kingdom

$119{ }^{(a)}$ INFN Sezione di Pavia; ${ }^{(b)}$ Dipartimento di Fisica, Università di Pavia, Pavia, Italy

120 Department of Physics, University of Pennsylvania, Philadelphia PA, United States of America

121 Petersburg Nuclear Physics Institute, Gatchina, Russia

$122{ }^{(a)}$ INFN Sezione di Pisa; ${ }^{(b)}$ Dipartimento di Fisica E. Fermi, Università di Pisa, Pisa, Italy

${ }^{123}$ Department of Physics and Astronomy, University of Pittsburgh, Pittsburgh PA, United States of America

124 (a) Laboratorio de Instrumentacao e Fisica Experimental de Particulas - LIP, Lisboa, Portugal;

(b) Departamento de Fisica Teorica y del Cosmos and CAFPE, Universidad de Granada, Granada, Spain

${ }^{125}$ Institute of Physics, Academy of Sciences of the Czech Republic, Praha, Czech Republic

126 Faculty of Mathematics and Physics, Charles University in Prague, Praha, Czech Republic

127 Czech Technical University in Prague, Praha, Czech Republic

128 State Research Center Institute for High Energy Physics, Protvino, Russia

129 Particle Physics Department, Rutherford Appleton Laboratory, Didcot, United Kingdom

130 Physics Department, University of Regina, Regina SK, Canada

131 Ritsumeikan University, Kusatsu, Shiga, Japan

$132{ }^{(a)}$ INFN Sezione di Roma I; ${ }^{(b)}$ Dipartimento di Fisica, Università La Sapienza, Roma, Italy

$133{ }^{(a)}$ INFN Sezione di Roma Tor Vergata; ${ }^{(b)}$ Dipartimento di Fisica, Università di Roma Tor Vergata, Roma, Italy

$134{ }^{(a)}$ INFN Sezione di Roma Tre; ${ }^{(b)}$ Dipartimento di Fisica, Università Roma Tre, Roma, Italy

$135{ }^{(a)}$ Faculté des Sciences Ain Chock, Réseau Universitaire de Physique des Hautes Energies - Université

Hassan II, Casablanca; ${ }^{(b)}$ Centre National de l'Energie des Sciences Techniques Nucleaires, Rabat;

${ }^{(c)}$ Faculté des Sciences Semlalia, Université Cadi Ayyad, LPHEA-Marrakech; ${ }^{(d)}$ Faculté des Sciences,

Université Mohamed Premier and LPTPM, Oujda; ${ }^{(e)}$ Faculté des sciences, Université Mohammed

V-Agdal, Rabat, Morocco

136 DSM/IRFU (Institut de Recherches sur les Lois Fondamentales de l'Univers), CEA Saclay

(Commissariat a l'Energie Atomique), Gif-sur-Yvette, France

137 Santa Cruz Institute for Particle Physics, University of California Santa Cruz, Santa Cruz CA, United States of America

138 Department of Physics, University of Washington, Seattle WA, United States of America

139 Department of Physics and Astronomy, University of Sheffield, Sheffield, United Kingdom

140 Department of Physics, Shinshu University, Nagano, Japan

141 Fachbereich Physik, Universität Siegen, Siegen, Germany

142 Department of Physics, Simon Fraser University, Burnaby BC, Canada

143 SLAC National Accelerator Laboratory, Stanford CA, United States of America

$144{ }^{(a)}$ Faculty of Mathematics, Physics $\&$ Informatics, Comenius University, Bratislava; ${ }^{(b)}$ Department of Subnuclear Physics, Institute of Experimental Physics of the Slovak Academy of Sciences, Kosice, Slovak Republic

${ }^{145}{ }^{(a)}$ Department of Physics, University of Johannesburg, Johannesburg; ${ }^{(b)}$ School of Physics, University of the Witwatersrand, Johannesburg, South Africa

$146{ }^{(a)}$ Department of Physics, Stockholm University; ${ }^{(b)}$ The Oskar Klein Centre, Stockholm, Sweden 
147 Physics Department, Royal Institute of Technology, Stockholm, Sweden

148 Departments of Physics 83 Astronomy and Chemistry, Stony Brook University, Stony Brook NY, United States of America

149 Department of Physics and Astronomy, University of Sussex, Brighton, United Kingdom

150 School of Physics, University of Sydney, Sydney, Australia

151 Institute of Physics, Academia Sinica, Taipei, Taiwan

152 Department of Physics, Technion: Israel Institute of Technology, Haifa, Israel

${ }^{153}$ Raymond and Beverly Sackler School of Physics and Astronomy, Tel Aviv University, Tel Aviv, Israel

154 Department of Physics, Aristotle University of Thessaloniki, Thessaloniki, Greece

155 International Center for Elementary Particle Physics and Department of Physics, The University of Tokyo, Tokyo, Japan

156 Graduate School of Science and Technology, Tokyo Metropolitan University, Tokyo, Japan

157 Department of Physics, Tokyo Institute of Technology, Tokyo, Japan

158 Department of Physics, University of Toronto, Toronto ON, Canada

$159{ }^{(a)}$ TRIUMF, Vancouver BC; ${ }^{(b)}$ Department of Physics and Astronomy, York University, Toronto ON, Canada

${ }^{160}$ Institute of Pure and Applied Sciences, University of Tsukuba,1-1-1 Tennodai, Tsukuba, Ibaraki 305-8571, Japan

161 Science and Technology Center, Tufts University, Medford MA, United States of America

162 Centro de Investigaciones, Universidad Antonio Narino, Bogota, Colombia

163 Department of Physics and Astronomy, University of California Irvine, Irvine CA, United States of America

$164{ }^{(a)}$ INFN Gruppo Collegato di Udine; ${ }^{(b)}$ ICTP, Trieste; ${ }^{(c)}$ Dipartimento di Chimica, Fisica e Ambiente, Università di Udine, Udine, Italy

165 Department of Physics, University of Illinois, Urbana IL, United States of America

166 Department of Physics and Astronomy, University of Uppsala, Uppsala, Sweden

167 Instituto de Física Corpuscular (IFIC) and Departamento de Física Atómica, Molecular y Nuclear and Departamento de Ingeniería Electrónica and Instituto de Microelectrónica de Barcelona (IMB-CNM), University of Valencia and CSIC, Valencia, Spain

168 Department of Physics, University of British Columbia, Vancouver BC, Canada

169 Department of Physics and Astronomy, University of Victoria, Victoria BC, Canada

${ }^{170}$ Department of Physics, University of Warwick, Coventry, United Kingdom

171 Waseda University, Tokyo, Japan

172 Department of Particle Physics, The Weizmann Institute of Science, Rehovot, Israel

173 Department of Physics, University of Wisconsin, Madison WI, United States of America

174 Fakultät für Physik und Astronomie, Julius-Maximilians-Universität, Würzburg, Germany

175 Fachbereich C Physik, Bergische Universität Wuppertal, Wuppertal, Germany

${ }^{176}$ Department of Physics, Yale University, New Haven CT, United States of America

177 Yerevan Physics Institute, Yerevan, Armenia

178 Domaine scientifique de la Doua, Centre de Calcul CNRS/IN2P3, Villeurbanne Cedex, France

${ }^{a}$ Also at Laboratorio de Instrumentacao e Fisica Experimental de Particulas - LIP, Lisboa, Portugal

${ }^{b}$ Also at Faculdade de Ciencias and CFNUL, Universidade de Lisboa, Lisboa, Portugal

${ }^{c}$ Also at Particle Physics Department, Rutherford Appleton Laboratory, Didcot, United Kingdom

${ }^{d}$ Also at TRIUMF, Vancouver BC, Canada

e Also at Department of Physics, California State University, Fresno CA, United States of America

${ }^{f}$ Also at Novosibirsk State University, Novosibirsk, Russia

${ }^{g}$ Also at Fermilab, Batavia IL, United States of America

${ }^{h}$ Also at Department of Physics, University of Coimbra, Coimbra, Portugal

${ }^{i}$ Also at Department of Physics, UASLP, San Luis Potosi, Mexico

${ }^{j}$ Also at Università di Napoli Parthenope, Napoli, Italy

${ }^{k}$ Also at Institute of Particle Physics (IPP), Canada

${ }^{l}$ Also at Department of Physics, Middle East Technical University, Ankara, Turkey 
m Also at Louisiana Tech University, Ruston LA, United States of America

${ }^{n}$ Also at Dep Fisica and CEFITEC of Faculdade de Ciencias e Tecnologia, Universidade Nova de Lisboa, Caparica, Portugal

- Also at Department of Physics and Astronomy, University College London, London, United Kingdom

${ }^{p}$ Also at Group of Particle Physics, University of Montreal, Montreal QC, Canada

${ }^{q}$ Also at Department of Physics, University of Cape Town, Cape Town, South Africa

${ }^{r}$ Also at Institute of Physics, Azerbaijan Academy of Sciences, Baku, Azerbaijan

$s$ Also at Institut für Experimentalphysik, Universität Hamburg, Hamburg, Germany

${ }^{t}$ Also at Manhattan College, New York NY, United States of America

${ }^{u}$ Also at School of Physics, Shandong University, Shandong, China

${ }^{v}$ Also at CPPM, Aix-Marseille Université and CNRS/IN2P3, Marseille, France

w Also at School of Physics and Engineering, Sun Yat-sen University, Guanzhou, China

${ }^{x}$ Also at Academia Sinica Grid Computing, Institute of Physics, Academia Sinica, Taipei, Taiwan

y Also at Dipartimento di Fisica, Università La Sapienza, Roma, Italy

${ }^{z}$ Also at DSM/IRFU (Institut de Recherches sur les Lois Fondamentales de l'Univers), CEA Saclay (Commissariat a l'Energie Atomique), Gif-sur-Yvette, France

aa Also at section de Physique, Université de Genève, Geneva, Switzerland

ab Also at Departamento de Fisica, Universidade de Minho, Braga, Portugal

ac Also at Department of Physics and Astronomy, University of South Carolina, Columbia SC, United States of America

ad Also at Institute for Particle and Nuclear Physics, Wigner Research Centre for Physics, Budapest, Hungary

ae Also at California Institute of Technology, Pasadena CA, United States of America

af Also at Institute of Physics, Jagiellonian University, Krakow, Poland

ag Also at LAL, Université Paris-Sud and CNRS/IN2P3, Orsay, France

ah Also at Nevis Laboratory, Columbia University, Irvington NY, United States of America

ai Also at Department of Physics and Astronomy, University of Sheffield, Sheffield, United Kingdom

aj Also at Department of Physics, Oxford University, Oxford, United Kingdom

ak Also at Institute of Physics, Academia Sinica, Taipei, Taiwan

al Also at Department of Physics, The University of Michigan, Ann Arbor MI, United States of America

am Also at Discipline of Physics, University of KwaZulu-Natal, Durban, South Africa

* Deceased 